Portland State University

PDXScholar

\title{
A Comparative Study of the Performance of Various Image Analysis Methods for Dimensional Inspection with Vision Systems
}

Ralf Heinrich Koeppe

Portland State University

Follow this and additional works at: https://pdxscholar.library.pdx.edu/open_access_etds

Part of the Mechanical Engineering Commons

Let us know how access to this document benefits you.

\section{Recommended Citation}

Koeppe, Ralf Heinrich, "A Comparative Study of the Performance of Various Image Analysis Methods for Dimensional Inspection with Vision Systems" (1989). Dissertations and Theses. Paper 3930.

https://doi.org/10.15760/etd.5814

This Thesis is brought to you for free and open access. It has been accepted for inclusion in Dissertations and Theses by an authorized administrator of PDXScholar. Please contact us if we can make this document more accessible: pdxscholar@pdx.edu. 
AN ABSTRACT OF THE THESIS OF Ralf Koeppe for the Master of Science in Mechanical Engineering presented July 18, 1989.

Title: A Comparative study of the Performance of Various Image Analysis Methods for Dimensional Inspection with Vision Systems

APPROVED BY THE MEMBERS OF THE THESIS COMMITTEE:

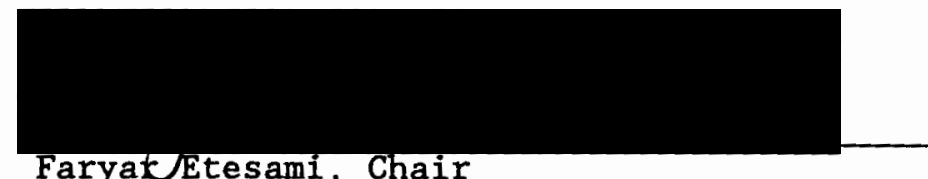
Faryatesami, Chair
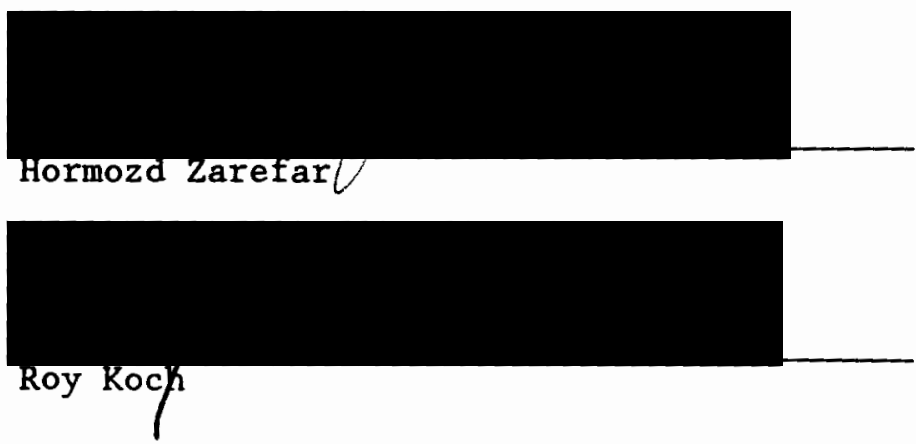

Dimensional inspection with Vision Systems requires a careful selection of image analysis methods in order to obtain accurate information about the geometry of the parts to be measured.

The purpose of this project is to study, implement and compare different image evaluation methods and to show their strengths and 
weaknesses with respect to dimensional inspection. Emphasis is made on the inspection of circular features. The criteria of comparison for these methods are discussed. Using synthetically generated images, various analysis methods are compared and conclusions for their use are drawn. Results of the comparison show that the selection of a method has to be done with regard to the noise level of the measurement. Finally, a computationally fast calibration algorithm is studied and implemented. 
A COMPARATIVE STUDY OF THE PERFORMANGE OF VARIOUS IMAGE ANALYSIS METHODS FOR DIMENSIONAL INSPECTION WITH VISION SYSTEMS

\author{
by
}

RALF HEINRICH KOEPPE

A thesis submitted in partial fulfillment of the requirements for the degree of

\author{
MASTER OF SCIENCE \\ in \\ MECHANICAL ENGINEERING
}

Portland State University

1989 
TO THE OFFICE OF GRADUATE STUDIES:

The members of the Committee approve the thesis of Ralf H. Koeppe presented July 18, 1989.

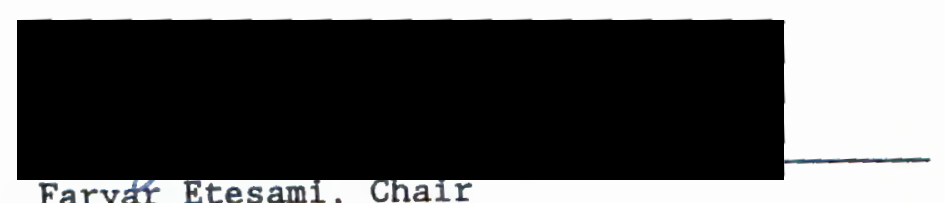

Faryát Etesami, chair
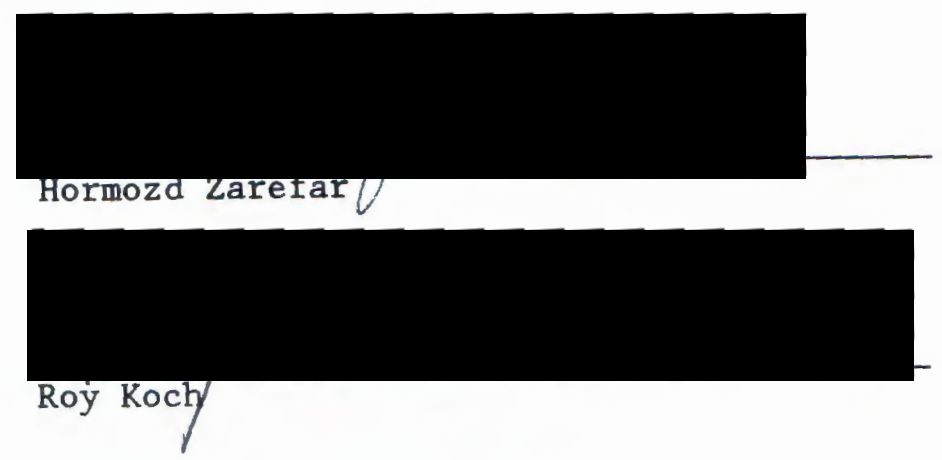

APPROVED:

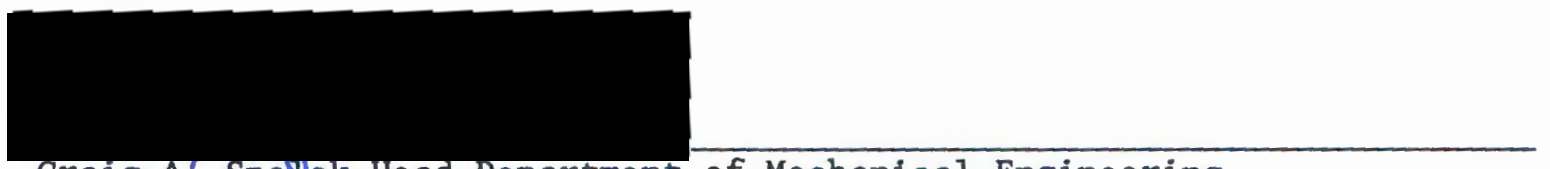

Graig A: Spoyek, Head, Department of Mechanical Engineering

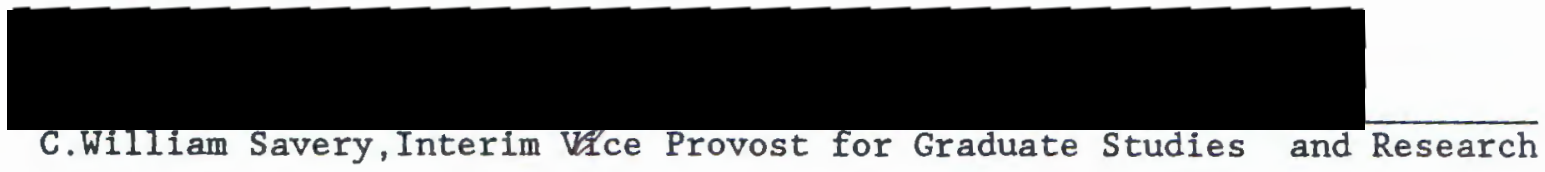




\section{ACKNOWLEDGEMENTS}

I wish to extend my warmest appreciations:

To Professor Faryar Etesami for his encouragement and guidance, and for giving me the opportunity to be creative;

To all faculty and staff members of the Department of Mechanical Engineering for their support;

To Dr. Rueff of the Institute of Manufacturing and Automatisation at the University of Stuttgart for his help and suggestions;

To my parents, Helga and Alfred, and my grandmother, Wilhelmine, for their love and support and for instilling in me the importance of education and helping me to achieve it;

And finally to Achim, André, Axel, Christina, Friedel, Raj, and Ray, and all my other friends in Portland, for their friendship.

Portland, Oregon

Ralf H. Koeppe 
TABLE OF CONTENTS

PAGE

ACKNOWLEDGEMENTS

LIST OF TABLES

vi

LIST OF FIGURES

\section{CHAPTER}

I INTRODUCTION $\ldots \ldots \ldots \ldots \ldots \ldots \ldots \ldots \ldots \ldots \ldots \ldots \ldots \ldots \ldots \ldots$

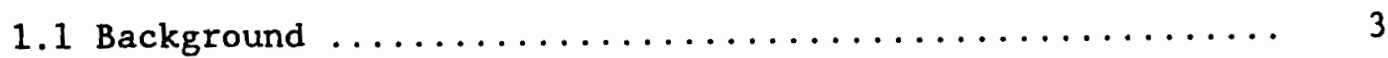

1.2 objectives $\ldots \ldots \ldots \ldots \ldots \ldots \ldots \ldots \ldots \ldots \ldots \ldots \ldots \ldots$

II DIGITAL IMAGE PROCESSING $\ldots \ldots \ldots \ldots \ldots \ldots \ldots \ldots \ldots \ldots$

2.1 Components of a Digital Image Processing System ...... 7

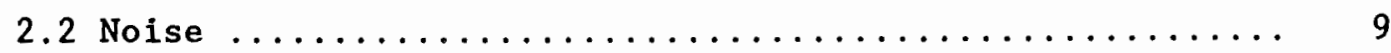

2.3 Dimensional Inspection with Vision systems $\ldots \ldots \ldots \ldots 14$

2.4 Image Processing and Analysis Methods ........... 15

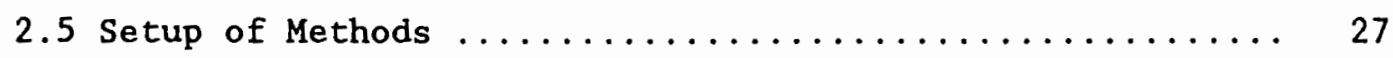

III COMPARISON OF IMAGE ANALYSIS METHODS $\ldots \ldots \ldots \ldots \ldots \ldots$

3.1 Measures of Performance $\ldots \ldots \ldots \ldots \ldots \ldots \ldots \ldots \ldots \ldots \ldots \ldots$

3.2 Decision Model $\ldots \ldots \ldots \ldots \ldots \ldots \ldots \ldots \ldots \ldots \ldots \ldots \ldots \ldots \ldots$

3.3 Image Generator $\ldots \ldots \ldots \ldots \ldots \ldots \ldots \ldots \ldots \ldots \ldots \ldots \ldots \ldots$

3.4 Simulation Mode1 ..................... 41

3.5 Simulation Test Results $\ldots \ldots \ldots \ldots \ldots \ldots \ldots \ldots \ldots \ldots \ldots \ldots$

3.6 Real Image Experiments ................... 54

3.7 statement of Comparison $\ldots \ldots \ldots \ldots \ldots \ldots \ldots \ldots \ldots \ldots \ldots \ldots \ldots$ 
IV CALIBRATION

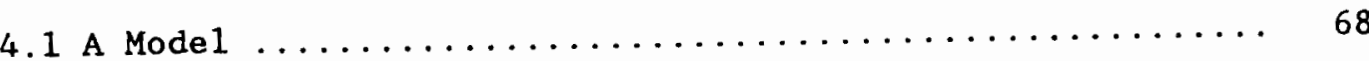

4.2 Computing the Calibration Parameters ............. 77

$\mathrm{V}$ CONCLUSIONS AND RECOMMENDATIONS $\ldots \ldots \ldots \ldots \ldots \ldots \ldots \ldots \ldots$

5.1 Conclusions $\ldots \ldots \ldots \ldots \ldots \ldots \ldots \ldots \ldots \ldots \ldots \ldots \ldots . \ldots \ldots$

5.2 Recommendations $\ldots \ldots \ldots \ldots \ldots \ldots \ldots \ldots \ldots \ldots \ldots, 83$

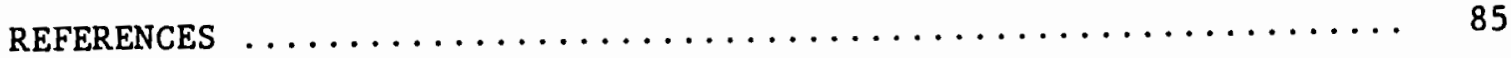

APPENDICES

A: THE PROGRAM SOURCE CODE OF THE LOCAL STATISTIC METHOD ...

B: THE PROGRAM SOURCE CODE OF

THE CONTOUR FOLIOWING ALGORITHM $\ldots \ldots \ldots \ldots \ldots \ldots \ldots, 93$

C: THE PROGRAM SOURCE CODE OF

THE AUTOMATED THRESHOLD SELECTION ALGORITHM $\ldots \ldots \ldots \ldots$.

D: THE PROGRAM SOURCE CODE OF

THE RADIUS ESTIMATION ALGORITHM................ 103

E: THE PROGRAM SOURCE CODE OF THE IMAGE GENERATOR $\ldots \ldots \ldots \ldots 106$

F: THE PROGRAM SOURCE CODE OF THE CALIBRATION ALGORITHM ... 112

G: THE INPUT PARAMETERS FOR EACH METHOD

AND SIMULATION CASE $\ldots \ldots \ldots \ldots \ldots \ldots \ldots \ldots \ldots \ldots \ldots \ldots \ldots$ 


\section{I.IST OF TABLES}

TABLE

PAGE

I Types of Noise $\ldots \ldots \ldots \ldots \ldots \ldots \ldots \ldots \ldots \ldots \ldots \ldots \ldots \ldots$

II Pixel Intensity Variation for Different Techniques

Standard Deviation and (Range) of 100 Samples .......... 12

III Quantitative Test Results of Low Contrast Simulation ...... 44

IV Quantitative Test Results of High Contrast Simulation ..... 45

V Qualitative Test Results of Low Contrast simulation ....... 51

VI Qualitative Test Results of High Contrast Simulation ...... 51 
LIST OF FIGURES

FIGURE

1. Automatic Dimensional Inspection System

Tolerance Verification $\ldots \ldots \ldots \ldots \ldots \ldots \ldots \ldots \ldots \ldots$

2. Components of a Digital Image Processing System ......... 8

3. Intensity Distribution of Pixe1 due to Noise .......... 13

4. Intensity Variation for 100 Samples - Comparison of Techniques 13

5. Mathematical Framework of Image Analysis Methods ......... 15

6. Roberts Edge Filter ........................ 19

7. Filtering of Image Noise using Local statistic ......... 20

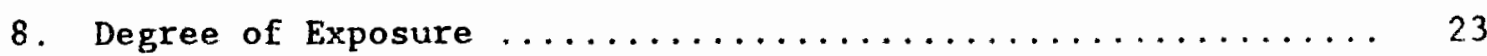

9. Automated Threshold Selection ................... 25

10. Transformation into Geometric space $\ldots \ldots \ldots \ldots \ldots \ldots$

11. Thresho1d (a) Method ....................... 29

12. Threshold (b) Method ...................... 30

13. Roberts Edge Filter Method .................... 31

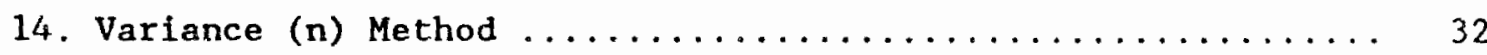

15. Local statistic Method ..................... 33

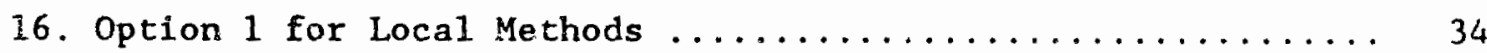

17. Option 2 for Local Methods .................. 35

18. Decision Mode1 .......................... 39

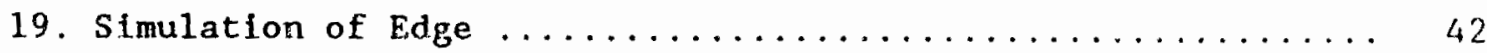

20. Pixe1 Stability - Low Contrast $1 \ldots \ldots \ldots \ldots \ldots$

21. Pixel Stability - Low Contrast $2 \ldots \ldots \ldots \ldots \ldots$ 
22. Geometric Stability - Low Contrast $1 \ldots \ldots \ldots \ldots$

23. Geometric Stability - Low Contrast $2 \ldots \ldots \ldots \ldots \ldots$

24. Pixel Stability - High Contrast $1 \ldots \ldots \ldots \ldots \ldots \ldots$

25. Pixe1 Stability - High Contrast $2 \ldots \ldots \ldots \ldots \ldots$

26. Geometric Stability - High Contrast $1 \ldots \ldots \ldots \ldots \ldots$

27. Geometric Stability - High Contrast $2 \ldots \ldots \ldots \ldots$

28. Gray Scale Image of Brass Cylinder with Textured Surface ... 56

29. Contour Image Extracted by the Threshold (a) Method ....... 57

30. Contour Image Extracted by the Local Statistic Method ...... 57

31. Contour Image Extracted by the Variance (5) Method ........ 58

32. Contour Image of Edge Detected by Roberts Edge Filter ...... 58

33. Contour Image Extracted by the Threshold (b) Method ....... 59

34. Gray Scale Image of Rectangular-shaped Block of Wood ...... 60

35. Contour Image Extracted by the Threshold (a) Method ....... 61

36. Contour Image Extracted by the Local Statistic Method ...... 61

37. Contour Image Extracted by the Variance (3) Method ....... 62

38. Contour Image Extracted by the Variance (5) Method ....... 62

39. Contour Image Extracted by the Variance (7) Method ....... 63

40. Contour Image of Edge Detected by Roberts Edge Filter ..... 63

41. Contour Image Extracted by the Thresho1d (B) Method ....... 64

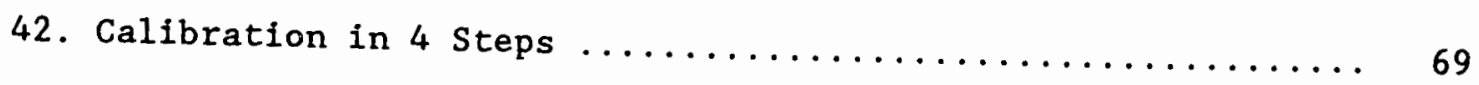

43. Orientation of Coordinate system for Calibration ......... 70

44. Scaling and Transformation into Computer Coordinate System .. 76

45. Accuracy of Vision Systems for Dimensional Inspection ..... 81

46. Resolution of Circle in Binary space $\ldots \ldots \ldots \ldots \ldots \ldots \ldots$ 
CHAPTER I

\section{INTRODUCTION}

One research focus at Portland State University is Geometric Modeling and Tolerancing and Computer Aided Design. Recent research has been done on the theory of assembly verification of features by simulation or soft-gaging [1]. For the assembly verification process, design specification data has to be matched with feature data of the manufactured parts. The design specifications are obtained by using a solid model or a 2-dimensional drafting model of a design in a CAD Systems. "Real World Data" of the manufactured part can be measured with the aid of coordinate measuring machines or vision systems. Where the former involves high cost and slow processing time but highly accurate results, the latter method allows faster processing time and lower cost.

Automated inspection with vision systems is being used in several industrial fields for the purpose of quality control. For example, the electronics industry uses vision systems to inspect miniaturized electronic components, which are beyond human inspection capability. Other systems are used to inspect automobile parts, metal surfaces, or food and packaging goods [2].

With the development of high resolution cameras and more powerful computers, vision systems are now used for dimensional inspection. The camera of the vision system takes an image of the part, which is then evaluated by image analysis and image processing methods. Geometric 
parameters, such as diameter, length, width, and tolerances are calculated and then compared to the specification of the parts. The requirements for vision systems for dimensional inspection are high speed, flexibility, and high accuracy [3]. The computation time to process the image and to extract the required data must be high enough to match the speed of the production line. The system has to be flexible enough to be adjusted to a wide range of measurement conditions, such as lighting or individual characteristics of the part. Finally, vision systems have to be accurate enough to accomplish the task of dimensional inspection. Some systems use high magnification lenses, combined with accurate positioning tables, to obtain the required resolution for measurements with high accuracy [4][5]. However, for a lot of parts with large dimensions, the use of positioning tables is not appropriate. The entire feature of the part to be measured has to appear in the image, and therefore the resolution of these systems is lower than of the ones described above. This leads to the following questions: 1) How accurate can vision systems be without using positioning tables? 2) What requirements must such a system meet? and 3) Which methods of analyzing images must be applied to obtain satisfying results? Figure 1 shows such a system.

A part on a conveyor belt is transported below the camera. An image is acquired and the information about dimensions and tolerances of the part are extracted and processed by the frame grabber and edge detector. The features of the part are sampled and compared to the specification. 


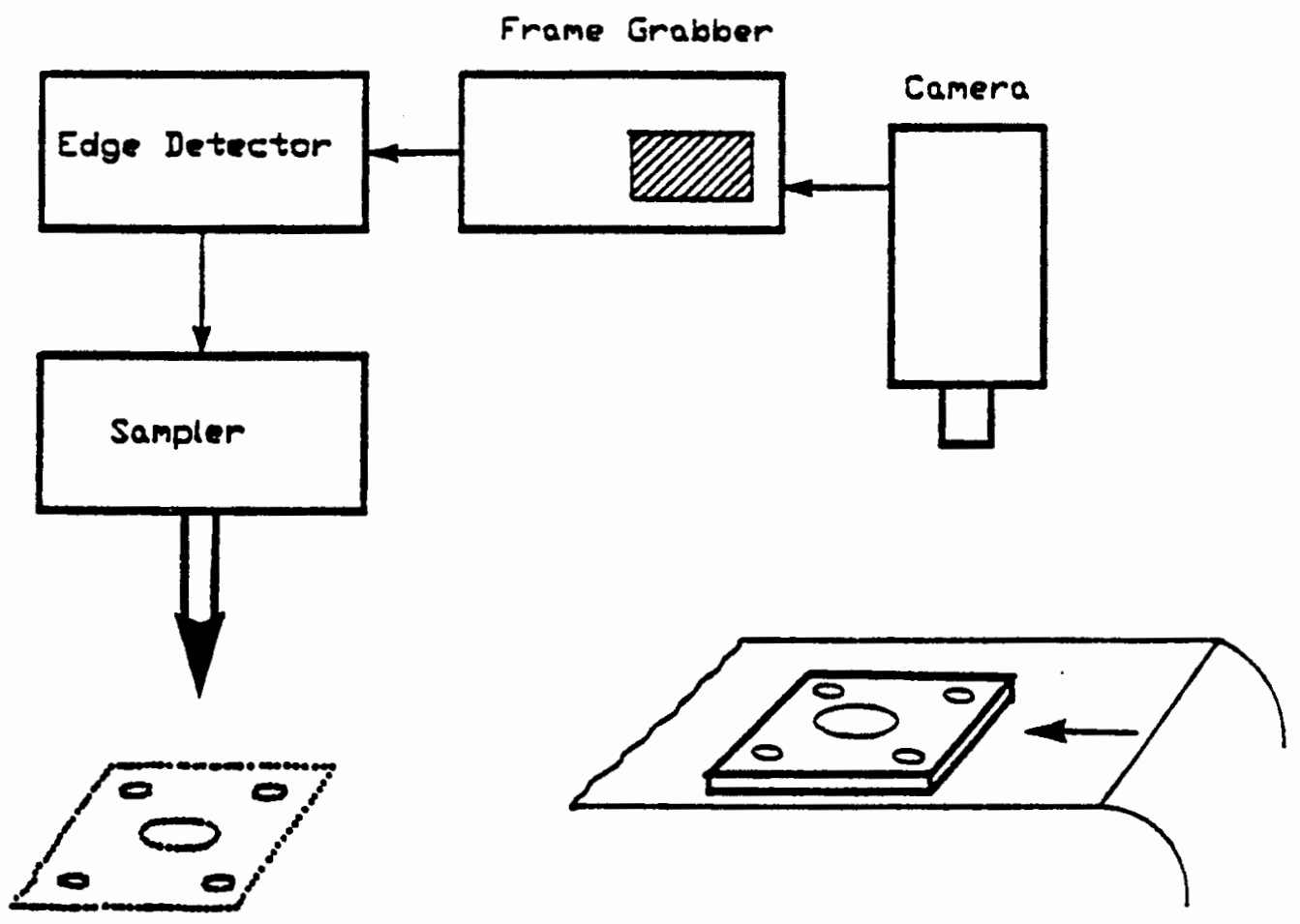

Figure 1.Automatic dimensional inspection system for tolerance verification.

\subsection{BACKGROUND}

Fundamental research has been done in image analysis and pattern recognition in the field of artificial intelligence. During the past 20 years, the use of vision systems has increased drastically and research in theoretical methods to extract information from the image has been strongly emphasized. Many comprehensive books have been published, and the newest achievements of research are discussed primarily in the journals, "Computer Vision, Graphics and Image Processing," "Pattern Recognition," "IEEE Transactions on Pattern Analysis and Machine Intelligence."

One main subject in this field is image processing. From a given scene, the two dimensional image representation is analyzed. One type 
of image analysis method extracts features such as edges, others label, organize, and manipulate this detected information. The former methods are called image enhancement and edge detection methods. Image enhancement is the field of manipulating the contrast of an image, and smoothing and sharpening it. This can be done within the spatial domain or the frequency domain. The purpose of the edge detection methods is to extract features of an image.

The original images consist of a two dimensional area represented in image points called pixels. Using modern equipment, every pixel can assume 256 different gray values. This gray scale image can be converted into binary space, which results in a black and white image. Due to the complexity of the subject, a lot of research is still being done in developing methods for image analysis. References [3] [6] - [8] provide insight into the use of image analysis methods, and are highly recommended for reading.

Related to the project are the publications by Peli and Malah [9], and Vliet, Young and Backers [10]. In both works, edge detection algorithms and the measures of comparing them are studied on circular features. However, this research and others focus on the general behavior of these algorithms. To determine how accurate and applicable image processing methods are for dimensional inspection with vision systems, the algorithms have to be implemented and tested for this specific task.

This project investigates the use of some fundamental image analysis methods, with regard to the measurement of circular features and their parametrization into geometric space. Among other works in 
the field of image processing methods applied the application dimensional inspection, the publication by Rueff and Melchior [11] in the building of a software library is recommended for study. Another interesting work has been done by Petkovic, Niblack and Flickner [12] in developing high accuracy methods for the measurement of straight line edges .

Because of the enthusiasm and productivity of the research community in the field of artificial intelligence, it is necessary for engineers concerned with the application of image analysis methods to be up-to-date. New or improved algorithms have to be implemented, applied and compared for the special task of a specific application.

\subsection{OBJECTIVES}

As previously mentioned, the purpose of this project is an investigation of the application of image analysis methods for dimensional inspection with vision systems. Emphasis is made on the analysis of circular features. Operations in the spatial domain are investigated. Among the selected methods are: thresholding, in other words, the conversion from gray scale into binary images using different mapping operations; pixel gradient and variance methods, measuring the change of the gray values within an image; and methods of improving the image quality by preprocessing it. Image enhancement operations in the frequency domain are not considered in this work. The reason for this is that these operations can be similarly performed in spatial domain.

The image noise due to low image quality and variability of the 
vision system is studied. Measures for comparing methods are defined. Using synthetical images, the methods are compared and the results are analyzed. These results are supported by applying and comparing these methods on real world images. Recommendations towards the use of image analysis methods are made.

To use vision systems for measurements, the system needs to be calibrated. A calibration model is studied and implemented. This calibration technique considers camera position and orientation, and lens distortion effect.

Finally, a model is proposed which shows that the image analysis method, the calibration model, and the resolution of a vision system determine its accuracy. 
CHAPTER II

DIGITAL IMAGE PROCESSING

\subsection{COMPONENTS OF A DIGITAL IMAGE PROCESSING SYSTEM}

Image signals are generated at a video source, for example, a Charge Couple Devices (CCD) camera. Charge Couple Devices consist of light sensitive elements lined up in rows and columns. As soon as light illuminates an element, an analog signal is generated. The analog signal is then transformed to a digital format. In the digitalization process, samples of the analog signal at discrete time intervals are taken. The image is stored in the frame memory as a 2 - dimensional array, with one array element called a pixel. Each pixel is stored by an 8-bit value, which corresponds to 256 possible gray values. The frame memory is connected to the CPU of a microcomputer. By using software implemented on the microcomputer, several operations can be performed on the image stored in the frame memory. The image is displayed on a monitor by backtransforming the digital information using a display logic. Figure 2 shows how different components of an image processing system are connected.

\section{Experiment Equipment for Digital Image Processing}

The image processing equipment used in this research project consists of a PC-AT with a PCVISIONplus Frame Grabber [13], a digitizer, a CCD camera Pulnix TS - 545 and a display monitor. The 
frame memory size is $1024 \times 512$ pixels with 8 bits per pixel. Either two images with $512 \times 512$ pixels or one single image with $640 \times 480$ pixels can be stored. The experiments were performed using the single image option. The input and output channels contain eight Look-Up Tables (LUT) each. They can be programmed to perform point operations on pixels in real time. In addition, PCVISIONplus provides software to interact with the frame grabber. These subroutines are written in $C$ programming language and allow initialization, read-write operations, and the manipulation of the image. Software developed during this project is written in C, using Microsoft C 5.1.

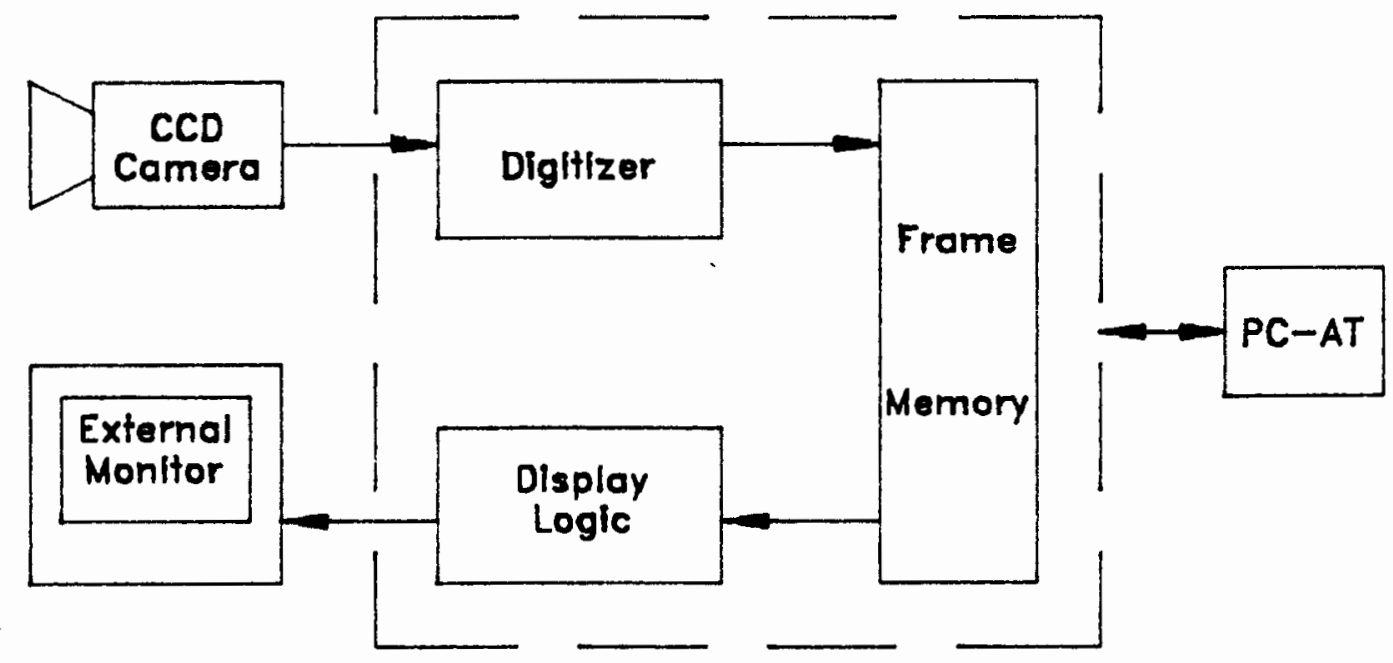

Figure 2.Components of a digital image processing system. 


\subsection{NOISE}

In the field of vision system and image processing, the term "noise" describes the phenomena of the information content of an image, which obstruct the extraction of the desired information. For example, due to sampling and quantization errors and the sensitivity of the Charge Couple Devices (CCD), the intensity of the pixels vary for the same image scene. This variation is called system noise. The distribution of the system noise can be approximated by a Gaussian curve [3].

Other types of noise and their origin are shown in Table I.

TABLE I

TYPES OF NOISE

\begin{tabular}{|l|l|l|}
\hline \multicolumn{2}{|c|}{ Origin } & \multicolumn{2}{c|}{ Type } \\
\hline System Noise & Hardware & $\begin{array}{l}\text { additive random normally } \\
\text { distributed with mean 0 }\end{array}$ \\
\hline Texture edge & Image & $\begin{array}{l}\text { can be simulated by random } \\
\text { normally distributed or by } \\
\text { binary noise }\end{array}$ \\
\hline Shadow & $\begin{array}{l}\text { Lighting } \\
\text { Conditions }\end{array}$ & \begin{tabular}{l} 
not random noise \\
\hline
\end{tabular}
\end{tabular}


Noise introduces variation in the image. Therefore images with high system noise and texture edges require special image analysis methods. The occurrence of shadows can be avoided by providing symmetrical illumination of the scene.

A measure of how much image information is distracted by noise is the Signal to Noise Ratio (SNR) [9], defined as

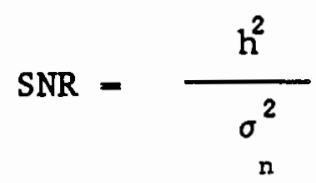

where $h$ is the heighth of the edge, and $\sigma_{n}$ the standard deviation of the noise. In the case of dimensional inspection the heighth of the edge can be approximated by

$$
\mathrm{h} \approx\left|\overline{\mathrm{I}}_{\mathrm{b}}-\overline{\mathrm{I}}_{\mathrm{o}}\right|
$$

where $\bar{I}_{b}$ is the average intensity of the background, and

$\bar{I}_{0}$ is the average intensity of the object.

For high contrast images the Signal to Noise ratio is SNR $-\rightarrow \infty$. For low contrast images SNR is a small value.

\section{Intensity Variation of Pixels}

To determine the variation in the intensity of an 8 bit pixel due to random system noise, and to investigate how image analysis techniques are effected by it, the following experiments were performed.

100 sample images of a cylindric black part on white background $(S N R=2500)$ were studied and the intensity values of one object, one background, and one edge pixel were captured. The standard deviation and the range of the intensity values were calculated. In addition, the intensity variation of pixels processed by Roberts edge filter, pixel 
averaging, and the mean and the variance of a $\mathrm{n} x \mathrm{n}$ neighborhood of the processed pixels, were recorded. (For description of these methods see the end of this chapter.)

The experiment results in Table II demonstrate that the image noise is approximately normally distributed with a mean of zero (Figure 3). The standard deviation of the intensity of edge pixels is higher than the standard deviation of the intensity of object or background pixels. By using image averaging, the intensity variation decreases. This agrees with statistic theory. The process of averaging reduces the standard deviation of the intensity of a pixel by $(\sqrt{n})^{-1}$. Therefore, this technique can be used as a noise filter for random system noise. Noise is also decreased by calculating the mean of a pixel within its $n \times n$ neighborhood (Figure 4). Whereas image averaging requires the use of multiple images of a stationary object, the calculation of the local mean can be done on one image. The intensity variation of pixels processed by the variance or the Roberts edge filter is shown in Table II. 
TABLE II

PIXEL INTENSITY VARIATION FOR DIFFERENT TECHNIQUES STANDARD DEVIATION AND ( RANGE ) OF 100 SAMPLES

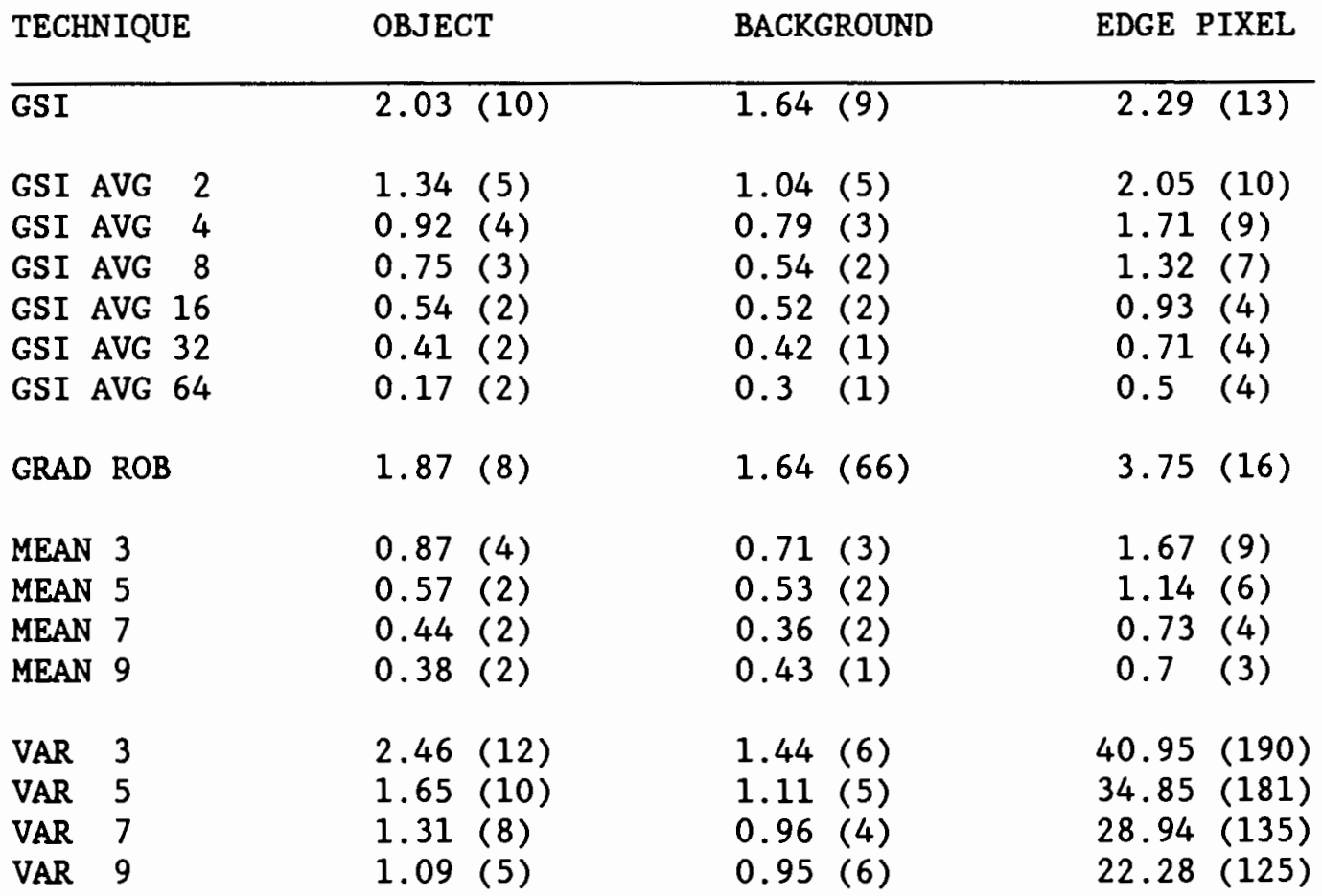

VARIATION OF THE PIXELS WITHOUT OBJECT

$\begin{array}{lrrrrr}\text { INTENSITY } & 145 & 1.56(9) & 1.25(5) & 1.45(7) \\ \text { INTENSITY } & 4 & 2.21(11) & 2.12(9) & 2.12(9)\end{array}$

$\begin{array}{ll}\text { GSI } & \text { Gray scale image } \\ \text { GSI AVG N } & \text { Gray scale image averaged } N \text { times } \\ \text { GRAD ROB } & \text { Gradient image using Roberts operator } \\ \text { MEAN N } & \text { Local mean with window size } N * N \\ \text { VAR N } & \text { Local variance with window size } N * N\end{array}$




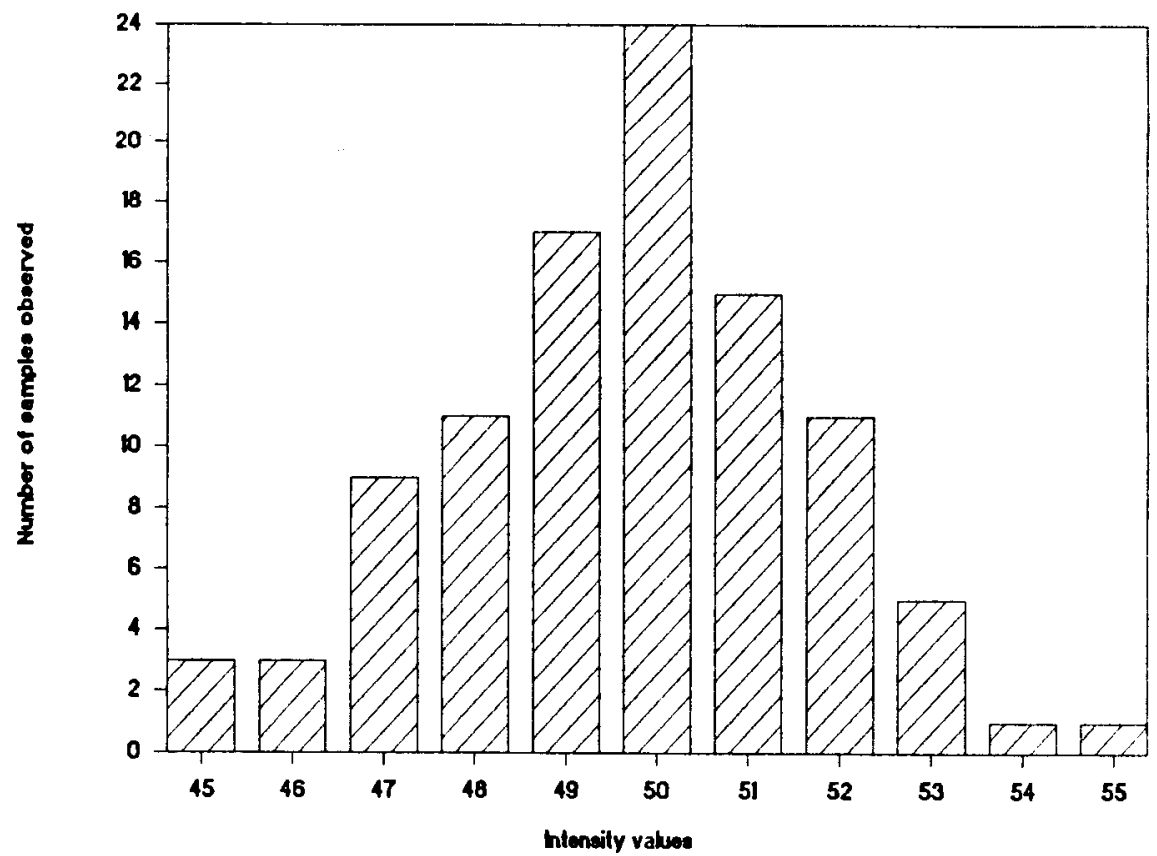

Figure 3.Intensity distribution of one pixel due to noise.

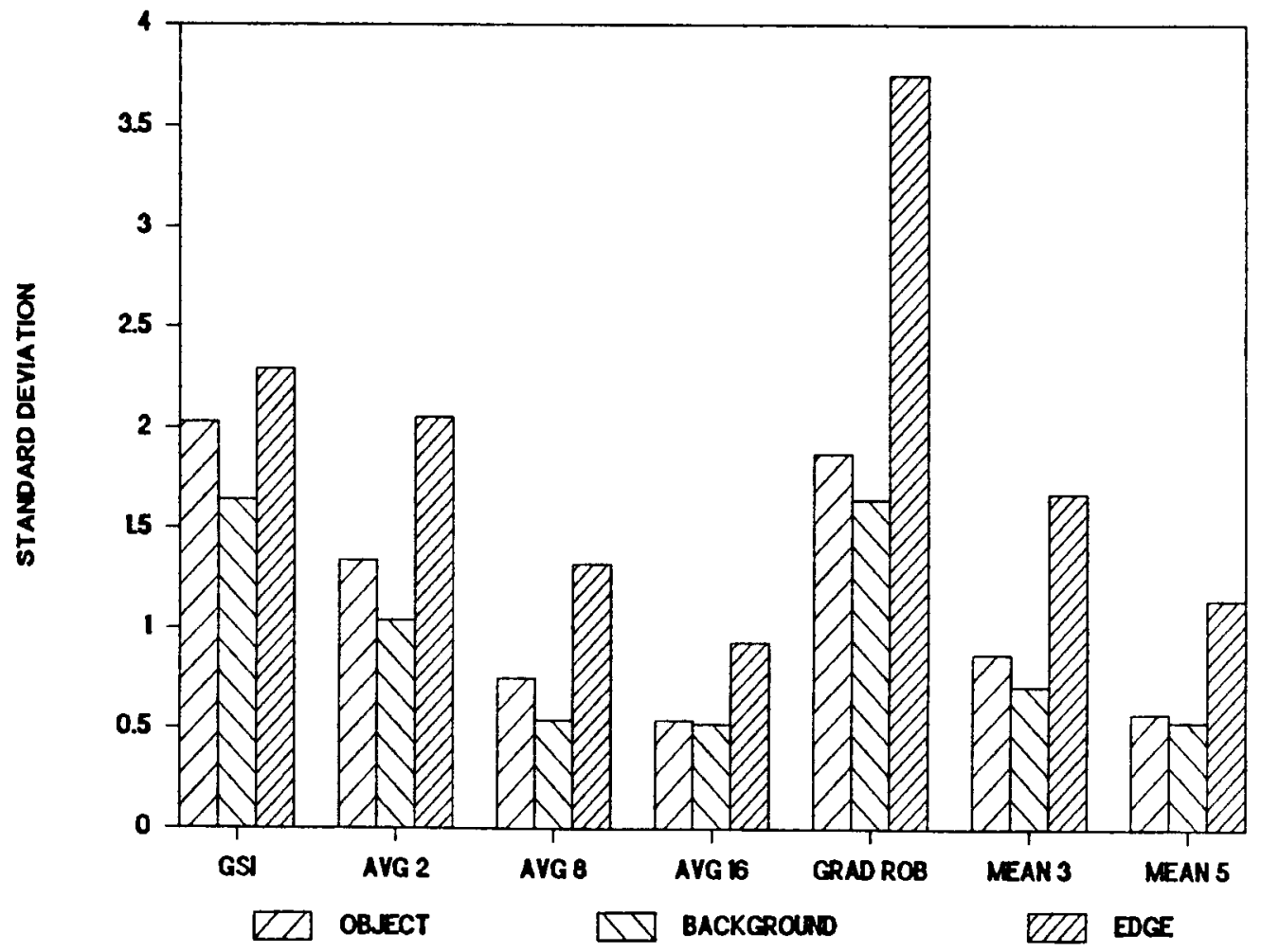

Figure 4. Intensity variation for 100 samples - comparison of techniques 


\subsection{DIMENSIONAL INSPECTION WITH VISION SYSTEMS}

The process of dimensional inspection with vision systems is divided into several steps. First, a gray-scale image is captured from a part to be inspected. Image processing methods then may improve the quality of the image by smoothing operations or noise filtering.

The next step is the process of edge detection. Depending on the method used, the decision whether or not a detected pixel might belong to an edge has to be done by thresholding. Displaying the enhanced edge pixels in a binary space results in a contour image of the part. In this stage, information about an edge is stored in the form of a set of $x$ and $y$ conrdinates of edgepoints in the digital coordinate system of the frame buffer.

For example, by the use of least square methods, the edge pixels, expressed in $x, y$ coordinates, are transformed into a geometrical representation of a circle.

The result of the dimensional inspection process is subject to errors. The errors are distinguished between systematic and statistical errors. Systematic errors are characterized by an assignable cause. The act of calibration described in Chapter IV compensates for such errors. Common systematic errors occur due to light variation or optical errors. Image noise in random form introduces statistical erfors. With the help of different image analysis methods it is possible to reauce noise of an image and to extract the edge of an object to be measured. 


\subsection{IMAGE PROCESSING AND ANALYSIS METHODS}

Rueff and Melchior [11] have developed an approach to organize and structure image analysis methods. The methods were divided into orthogonal and nonlinear transformations, point, local, and global operations, which are related in a mathematical framework (Figure 5.).

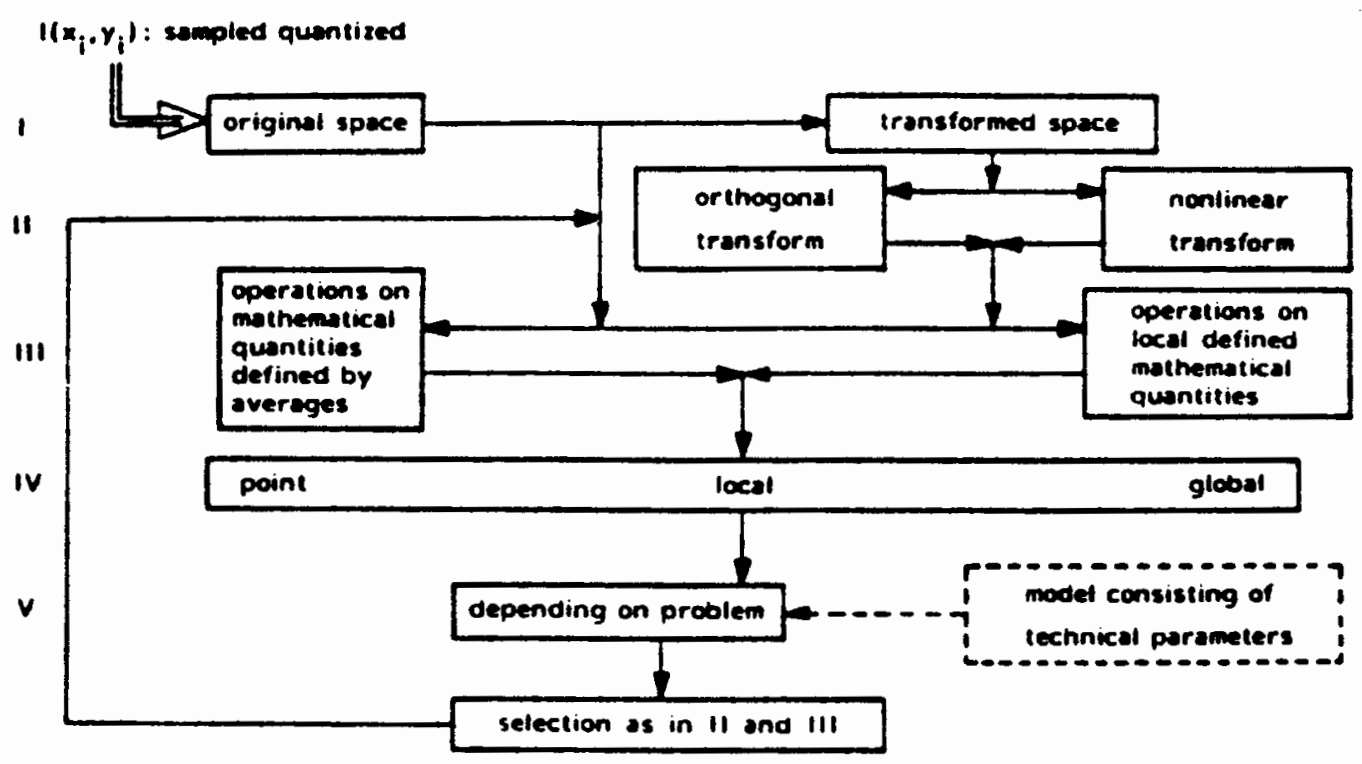

Figure 5.Mathematical framework of image analysis methods [11].

In this project, point, local, and global operations are investigated. Image processing in transformed space could be the subject of later research.

The following operations were studied and implemented in this project.

\section{Point Operations}

Threshold (a)

Threshold (b) - Highcut, Lowcut 
Inage Averaging

Local Operations

Variance Mask (3)

Variance Mask (5)

Roberts Edge Filter

Filtering of Image Noise using Local Statistics - Lee

Global Operetions

Contour Following

Automatic Th:eshold selection

Arc Center and Radius Estimation.

\subsubsection{Point Operations}

Point operations are mathematical operations on one pixel.

Threshold (a). Threshold (a) creates a binary image by using the following operation.

$N(x, y)$ e F intensity $(x, y)-\left\{\begin{array}{rr}\hat{1} & 0 \text {, intensity }(x, y)<\text { threshold } \\ 6 & 255 \text {, intensity }(x, y) \text {. threshold }\end{array}\right.$

where $F$ is the set of pixels stored in the Frame Buffer, and intensity $(x, y)$ e $(0.255)$.

Threshold (b) - Lowcut, Highcut. Threshold (b) creates a binary image by using the following operation. 


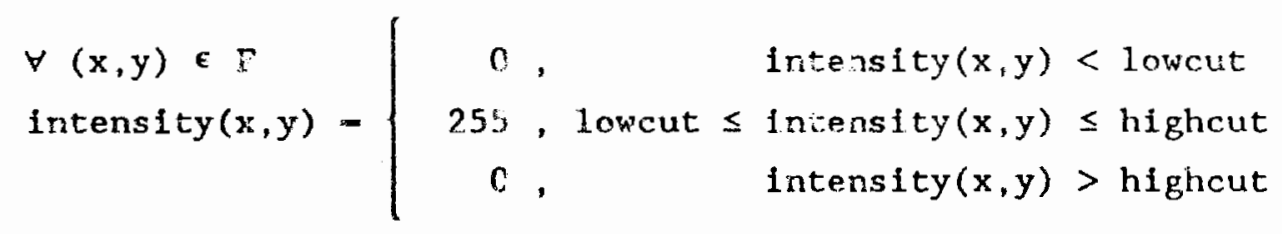

Threshold operations are chosen because they represent a classical method in the use of vision systems for dimensional inspection. The advantage of thresholding, in general, is that the transformation into a binary image is done by hardware, and therefore can be performed very fast.

Thresholding (b) is a vartant of method (a) by using high and lowcut threshold values and incorporates the advantage that the thickness of the contour, in other words the number of edge pixels, can be controlled.

Image Averaging. Image averaging of static images results in the reduction of the normal distributed random noise [14]. The intensity values of several images are comblned pixel by pixel and the mean is calculated. This simple image preprocessing method is chosen because of its capability to reduce noise without using local operators. Image averaging can be performed by the image processing software.

\subsubsection{Local Operations}

Local operations are mathematical operations on one pixel incorporating spatial relations. 
Variance (3)(5). Variance (n) calculates the variance of the intensity of a pixel in an $n \times n$ neighborhood as:

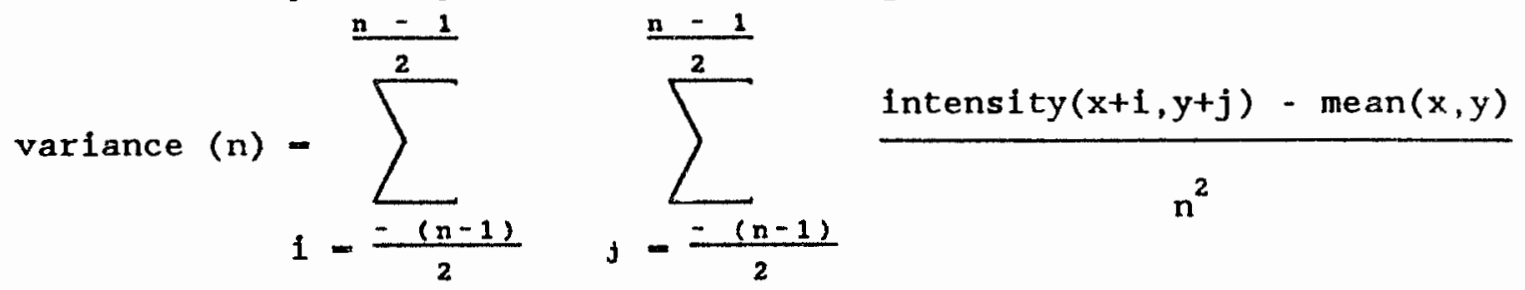

for $n \in(3,5,7, \ldots\}$, and mean $(x, y)$ the average intensity value of the nxn neigborhood.

The magnitude of the variance is a measure of the strength of an edge. Since an edge is a high contrast area, the magnitude of the variance of an edge pixel is high. The edge is defined as the set of pixels in which the variance value exceeds a given threshold.

\section{Roberts Edge Filter}

Roberts cross point edge filter belongs to the methods of gradient operators. Roberts [15] defines the magnitude of the gradient as the maximum of the differences of the intensity values between two crossed adjacent pairs of pixels (Figure 6). The Roberts gradient value for a pixel is defined as:

$$
\max \{|i(x, y)-i(x+1, y+1)|,|i(x, y+1)-i(x+1, y)|\}
$$

where the function $I(x, y)$ is the intensity $(x, y)$ of a pixel.

This well known edge detection method is chosen because in Peli and Malah's [9] comparison of edge detection algorithms, its performance was shown to be good on ramp edges, and on ideal edges superpositioned with Gaussian noise, for high signal to noise ratios. As in the previous method, the edge is determined by pixels with their gradient 
value exceeding a specified threshold.

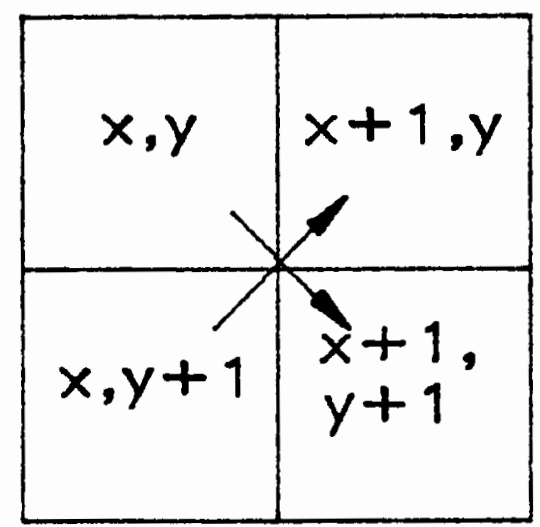

Figure 6.Roberts edge filter

\section{Filtering of Image Noise Using Local Statistics}

Lee [16][17] presented an algorithm for noise filtering by calculating mean and variance of a reduced subset of pixels incorporating gradient information (Figure 7). For every pixel, the intensity information of its $7 \times 7$ neighborhood is reduced to a $3 \times 3$ window by calculating the submeans of the 9 overlapping subareas of size $3 \times 3$ pixels. Within this subarea, the three-level simple mask of Robinson [18] is applied. A mask operation on a pixel intensity value is performed by replacing the intensity for a pixel with the new value

$$
\begin{aligned}
\operatorname{inew}(x, y) & =\text { w11 } i(x-1, y-1)+w 12 i(x-1, y)+w 13 i(x-1, y+1)+ \\
& + \text { w21 } i(x, y-1)+w 22 i(x, y)+\text { w23 } i(x, y+1)+ \\
& + \text { w31 } i(x+1, y-1)+w 32 i(x+1, y)+w 33 i(x+1, y+1)
\end{aligned}
$$

where w11 - w33 are the values of the matrix of the mask. 

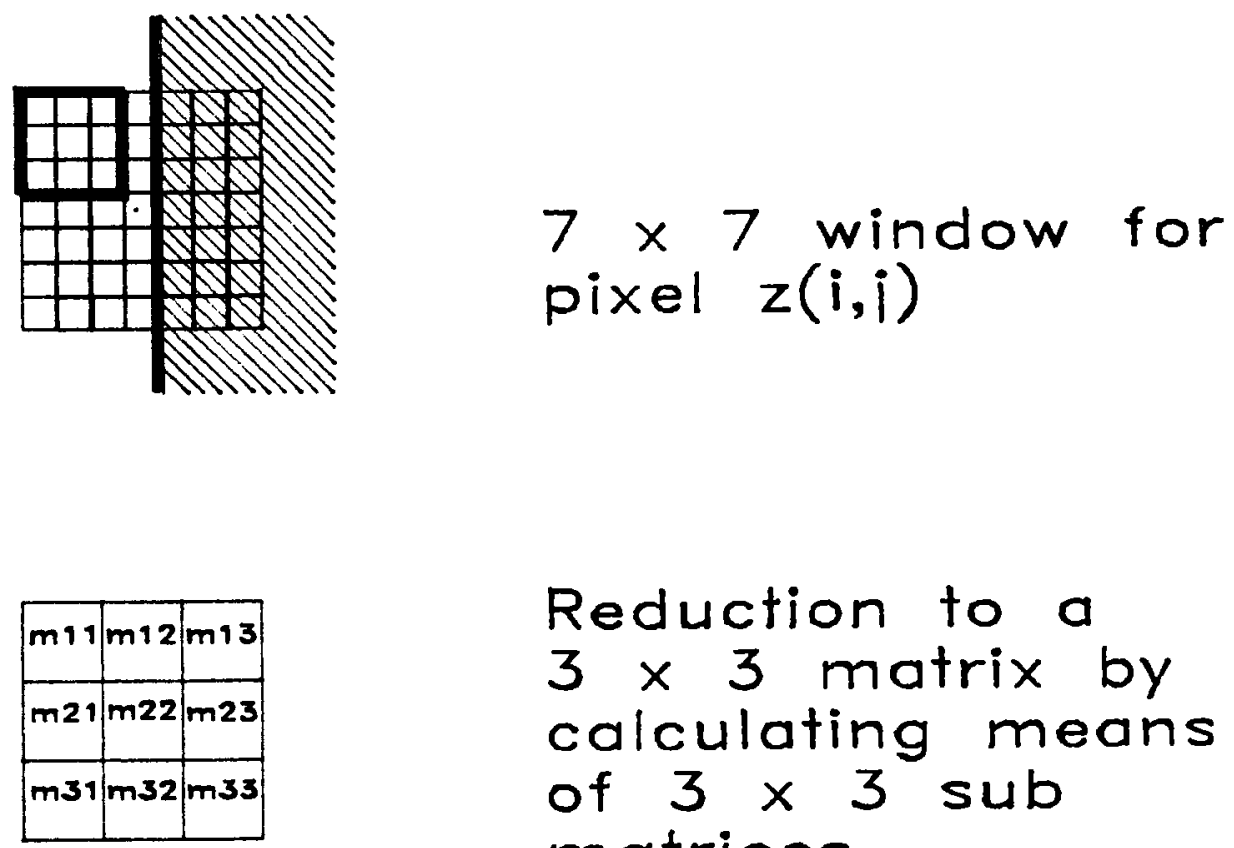

Reduction to a

$3 \times 3$ matrix by calculating means of $3 \times 3$ sub matrices

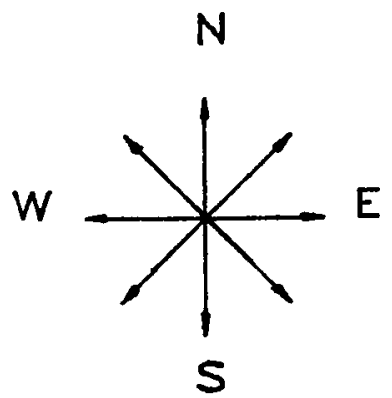

Calculation of gradient directions

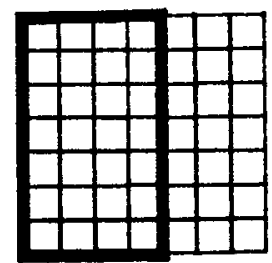

Calculation of mean and variance of submatrix

Replacement of pixel $z(i, i)$ with $x$ new $=$ $f($ mean, variance, $z)$ 
Example of Three-Level Simple Masks:

$$
\begin{aligned}
& \text { North }\left[\begin{array}{rrr}
1 & 1 & 1 \\
0 & 0 & 0 \\
-1 & -1 & -1
\end{array}\right] \\
& \text { Northwest } \\
& \left.\begin{array}{rrrr}
1 & 1 & 0 \\
1 & 0 & -1 \\
0 & -1 & -1
\end{array}\right]
\end{aligned}
$$

The Three-Level Simple mask detects edges in a specific direction, for example north, by calculating the gradient. Based on the gradient information obtained in the previous step, the subset which is the most likely to represent the center pixel of the $7 \times 7$ window is chosen. The mean and the variance are calculated from this subset. The center pixel is replaced by the new value calculated by

$$
\hat{x_{1, j}}-\bar{x}_{1, j}+\frac{Q_{1, j}}{Q_{1, j}+\sigma_{1}^{2}} \cdot\left(z_{1, j}-\bar{x}_{1, j}\right)
$$

where $\bar{x}_{1, j}$ is the mean of the subset of the $7 \times 7$ window;

$Q_{1, j}$ is the variance of the subset of the $7 \times 7$ window;

$z_{1, j}$ is the original intensity value of the center pixel;

$\sigma_{1}^{2}$ is the assumed noise variance.

For a low contrast area $x_{i, j}=\bar{x}_{i, j}$ and for a high contrast area for example an edge $x_{1, j}=z_{i, j}$

This algorithm is chosen because it represents the group of local operators with noise filtering capability by preprocesssing the image. The source code of the algorithm is included in Appendix A. 


\section{4 .3 Global Operations}

Contour Following. A contour following algorithm is capable of extracting closed contours in binary fmages of an object. Due to the built-in backtracking algorithm, it is possible to follow the contour of sharp or noisy edges. The essence of this algorithm is given below. The contour search process can be divided into four steps :

1. A window has to be specified that contains the contour to be formed

2. The Initial edge pixel of the object is searched in the first quacrant of the vindow. The coordinates are stored on the first position of an $x, y$ data array with length 0 . maxlength.

3. The next boundary pixel is found by searching a $3 \times 3$ neighborhood for a pixel. which must satisfy the following conditions :

- the intensity of the pixel $I\left(f_{x}, j_{y}\right)$ is equal to the intensity of the object;

- the degree of exposure (DOE) (Figure 8) is:

$$
\max \left[\operatorname{DOE}\left(i_{x}, j_{y}\right)\right], \forall\left(i_{x}, j_{y}\right) \in S \wedge 0<\operatorname{DOE}\left(i_{x}, i_{y}\right)<4
$$

- the pixel is not already found within the last a boundary points.

The degree of exposure (DOE) of a pixel $i_{x}, j_{y}$ is defined as the number of adjacent pixels with the intensity of the background. Where adjacent pixels of $(x, y)$ are specified as the set of points 
$S=\{(x+1, y) ;(x-1, y) ;(x, y+1) ;(x, y-1)\}$

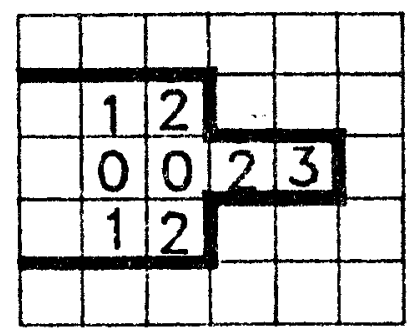

Figure 8, Degree of Exposure - The object pixel with number 3 is surrounded by 3 background pixels and therefore $\operatorname{DOE}(x, y)=3$ for this pixel.

4. The new boundary pixel is stored in the array of $x, y$ coordinates and the next boundary pixel has to be found. The condition to end the search is when the new boundary pixel is equal to the initial pixel or when the length of the data array exceeds the maximum specified length.

The following situations will require exceptional handling:

1. If the degree of exposure of the last boundary pixel in the array of edgepixels is $\mathrm{DOE}=3$, and all other pixels within the neighborhood are either already stored edge pixels or pixels with background intensity, the algorithm would not be capable of finding a new boundary pixel. Therefore a backtracking algorithm is incorporated. Backtracking: If no new boundary pixel is fourd, the backtracking algorithm searches within the last $n$ boundary points for the next boundary pixel. For this task, exactly the same procedures are used as in the forward tracking. The new boundary pixel found is stored at the $i+1$ th position where the baclitracking mechanist started. Another poss bility would be to 
skip the boundary points which were backtracked and to search on from the point where the new boundary pixel was found. This would result in smoothing the contour of the object.

2. Another problem occurs if in the search process for the initial pixe1, pixels with object intensity were found which do not belong to the object contour. Such situations occur with increasing nolse, exceeding the threshold. In this case, the algorithm continues searching for an initial boundary pixel of the object, or fails if the first quadrant is completely searched. The source code of this algorithm is given in Appendix $B$.

\section{Automated Threshold Selection}

To automate the task of thresholding gray level images into binary images, an algorithm proposed by Johannsen and Bille [19] was implemented (Figure 9).

By computing the Shannon's entropy of the gray level diagram, the gray levels are separated into two subsets, $G_{k}$ and $G_{k-1}$ so that the interdependence between them is minimized. The entropy of a distribution is defined as:

$$
H=-\sum_{k=1}^{n} p_{k} \cdot \ln p_{k}
$$<smiles></smiles>

where pk is the distribution of the intensity, Nk the number of pixels with the intensity $k$ in the image and $N=\Sigma \mathrm{Nk}$. 


\section{Probability Distribution}

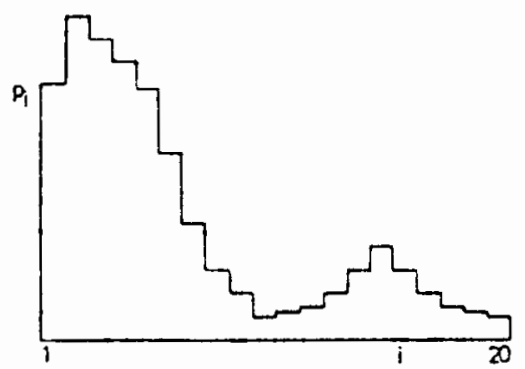

divide in subset $G k, \bar{G} k$ for all $k$ calculate $S k, \bar{S} k$ with $S$ as a

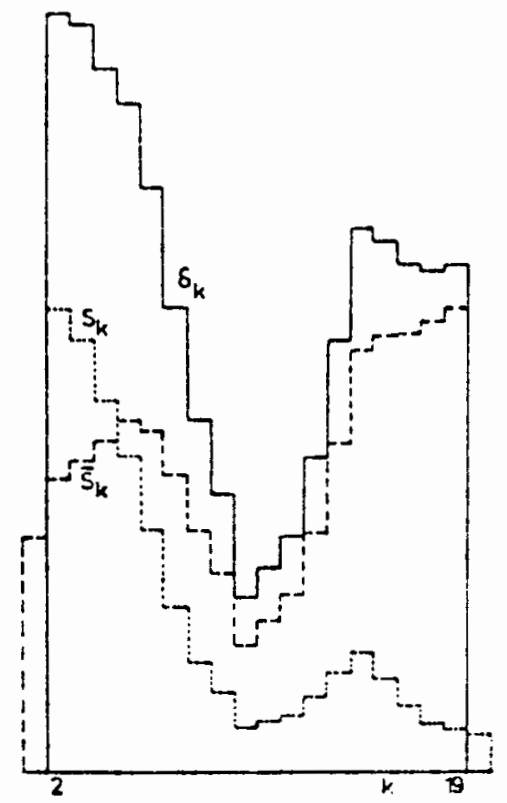
measure of the contribution of gray level $k$ to the part of the histogram composed of the divided subsets GK and $\bar{G} k-1$

$\operatorname{Min}($ delta $k)=\operatorname{Min}(S k+\bar{S} k)$

Threshold value $=k$

Figure 9.Automated threshold selection 
By minimizing the sum of the entropies $S_{k}$ and $\bar{S}_{k}$ of the divided subsets, where $S_{k}$ and $\bar{S}_{k}$ measure the contribution of a gray level $k$ to the part of the histogram composed of the divided subset $G_{k}$ and $G_{k-1}$, the threshold value $K$ can be determined by

$$
\begin{aligned}
& \min \left(\delta_{k}\right)=\min \left(S_{k}+\bar{S}_{k}\right), \\
& \text { where the threshold }=k
\end{aligned}
$$

This algorithm supports thresholding (a). The source code of the implemented program is included in Appendix $C$.

\section{Arc Center and Radius Estimation}

To transfer edge information into geometric representation, the pixel coordinates have to be processed by an estimation algorithm. In the case of circular features, the geometry of the object is described in diameter and $x, y$ coordinates of the center of the circle. The algorithm used for this purpose has been proposed by Thomas and Chan [20].

The error between a constant area $\pi \cdot R^{2}$ and the area of the circle centered at $\bar{x}, \bar{y}$ is minimized. This results in the equation

$$
e(R, \bar{x}, \bar{y})=\sum_{i=1}^{N}\left[\pi R^{2}-\pi \cdot\left\{\left(x_{i}-\bar{x}\right)^{2}+\left(y_{1}-\bar{y}\right)^{2}\right\}^{2}\right.
$$

in which its partial derivatives are set to zero to find the minimum.

$$
\frac{\partial e}{\partial R}=0 \quad \frac{\partial e}{\partial \bar{x}}=0 \quad \frac{\partial e}{\partial \bar{y}}=0
$$


This results into two linear equations. Using standard algebraic calculations handling summation terms and solving the two linear equations for the two unknowns $\bar{x}, \bar{y}$, the center of the estimated circle is calculated. Therefore, the radius yields

$$
\mathrm{R}^{2}=-\frac{1}{\mathrm{~N}}\left\{\sum \mathrm{x}_{1}^{2}-2 \overline{\mathrm{x}} \sum \mathrm{x}_{1}+\mathrm{Nx}^{2}+\sum \mathrm{y}_{1}^{2}-2 \mathrm{y} \sum \mathrm{y}_{1}+\mathrm{Ny}^{2}\right\}
$$

The source code is documented in Appendix D.

\subsection{SETUP OF METHODS}

For the process of dimensional inspection, the image operations, available in the form of software subroutines, are linked together. The point, local, and global operations are combined to evaluation algorithms, starting with the acquisition of an image and ending with the results of the measurement. The output image of an evaluation algorithm is a binary contour image of the object. At this stage, edge information is available in the form of $x, y$ coordinates of the frame buffer. By using estimation and least square methods, edge information is transformed into a geometric representation of the object. In the process of measurement of circular features, the $x, y$ coordinates are parametrized in terms of diameter and the center coordinates of the circle (Figure 10). The Figure 11 - 15 show the structure of the different evaluation algorithms, implemented for the experiments in this work. To avoid confusion, in the rest of this document the expression "method X" refers to the evaluation algorithm using the point or local "image analysis operation $\mathrm{X}$ ". 


\section{Binary \\ Contour Image}

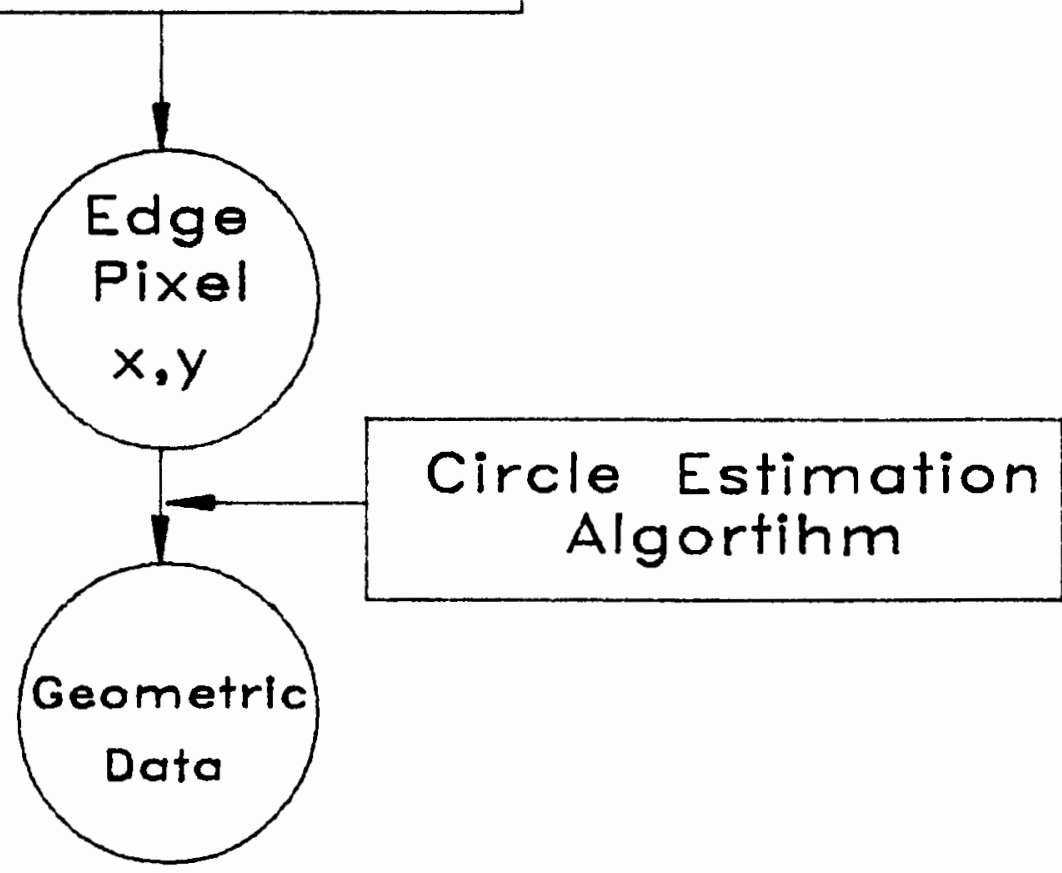

Figure 10.Transformation into geometric space 


\section{Threshold (a)}

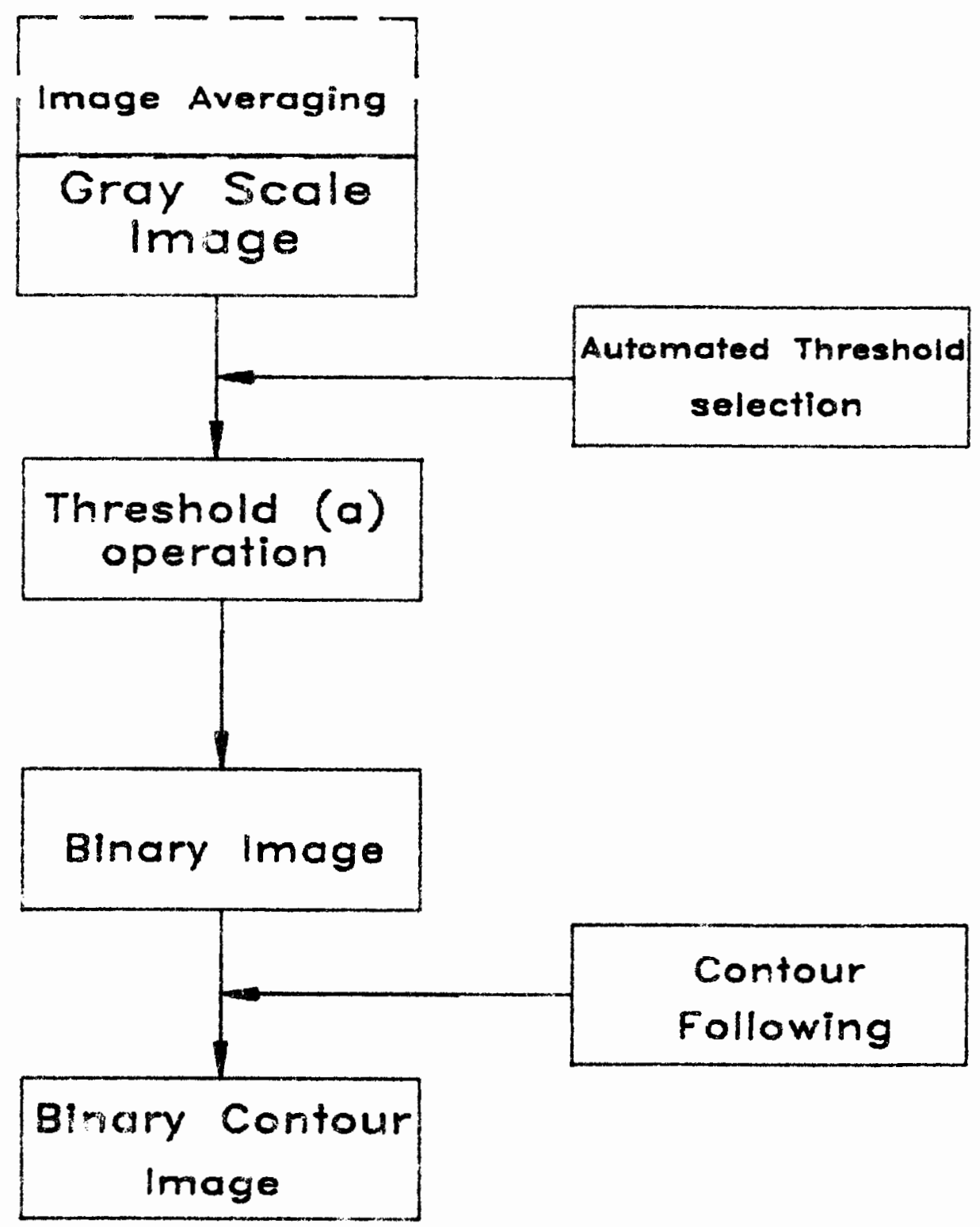

Figure 11.Tnreslold(a) method. The evaluation program acouires a gray scale image, including the option of image averaging. The automated threshold selection operation determines the threshold. By using the threshold(a) operation, a binary inage is obtained. The contour following algorithm extracts the edge pixels, which are displayed as a binary contour image. 


\section{Thireshold (b)}

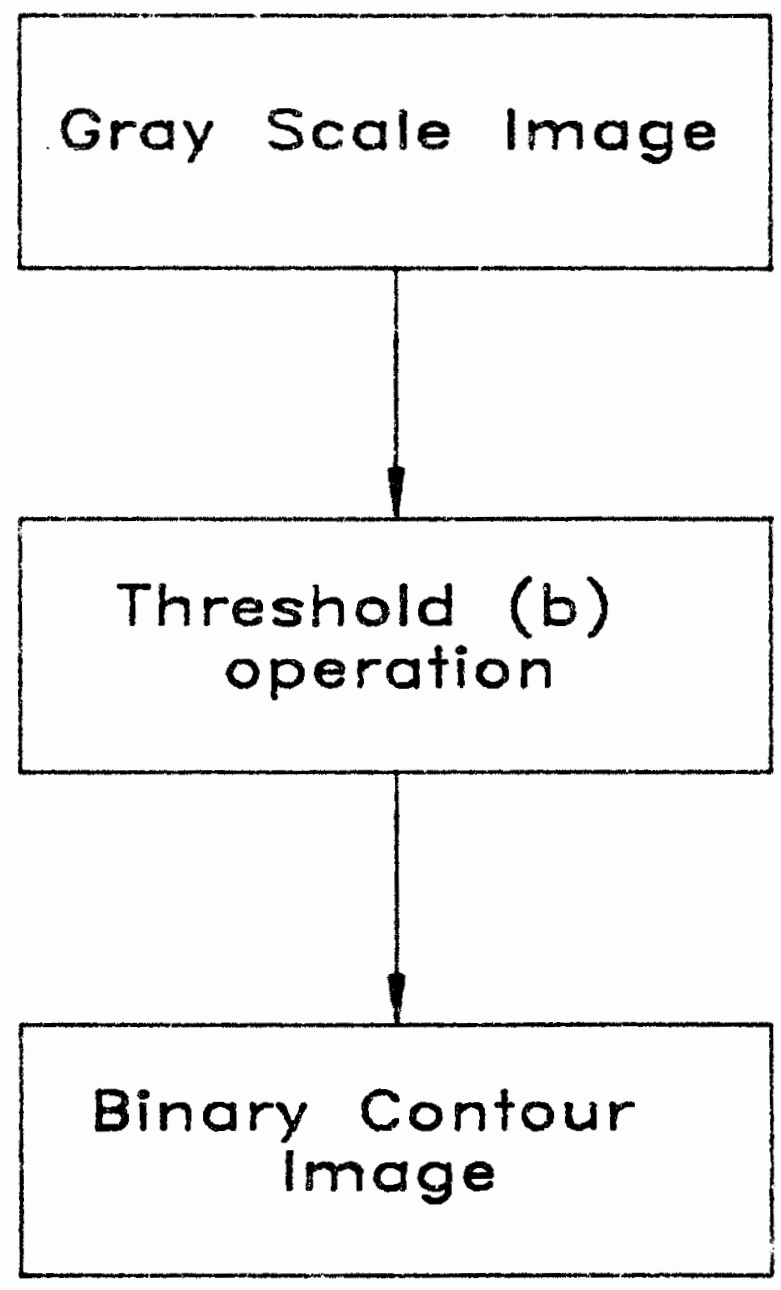

Figure 12.Threshold(b) method. The acquired gray scaled image is transformed into a binary contour image. Edge pixels are pixels with intensity: lowcut < intensity < highout. 


\section{Roberts Edge Filter}

\section{Gray Scale Image}

Roberts Edge Filter gradient operator

Gradient Threshold operation

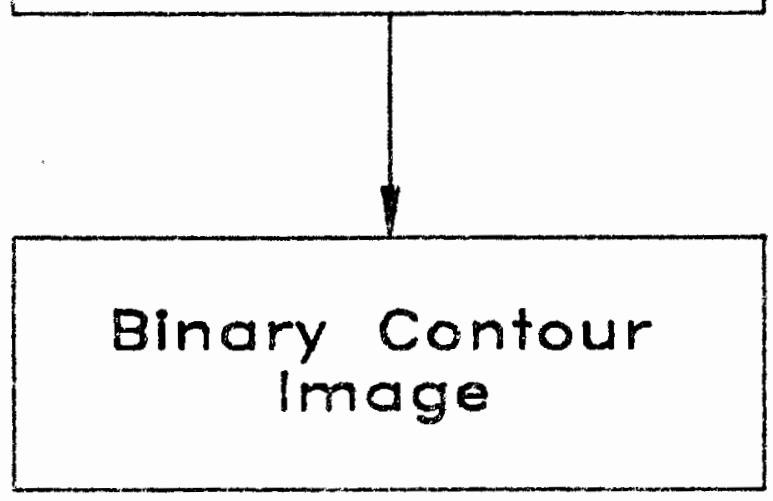

Figure 13. Roberts edge filter method. The gray scale inage is converted into a gradient image. Gradient values exceeding a specified threshold form the edge and are displayed in the binary contour image. 
Variance $(n)$

\section{Gray Scale Image}

\section{Variance operator $n \times n$}

Variance Threshold operation

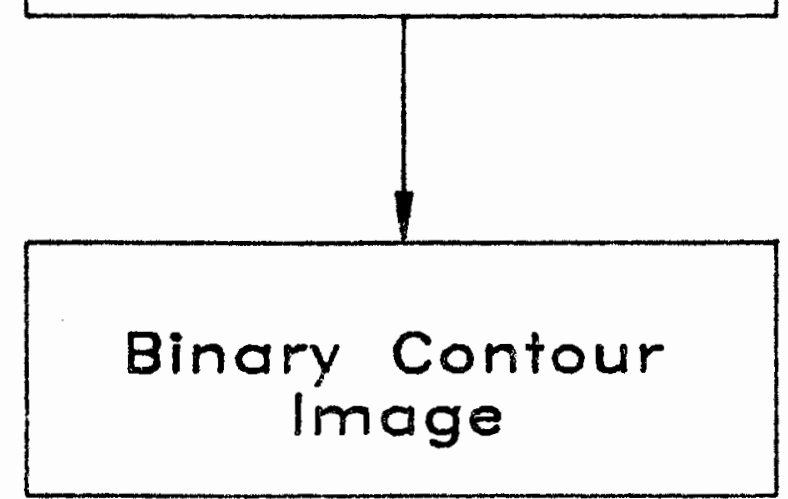

Figure 14.Variance(n) method. Similar to Roberts edge filter, the edge pixels of variance (n) are pixels exceeding a specified variance threshold. 
Filtering of image noise using Lee's Local Statistic method

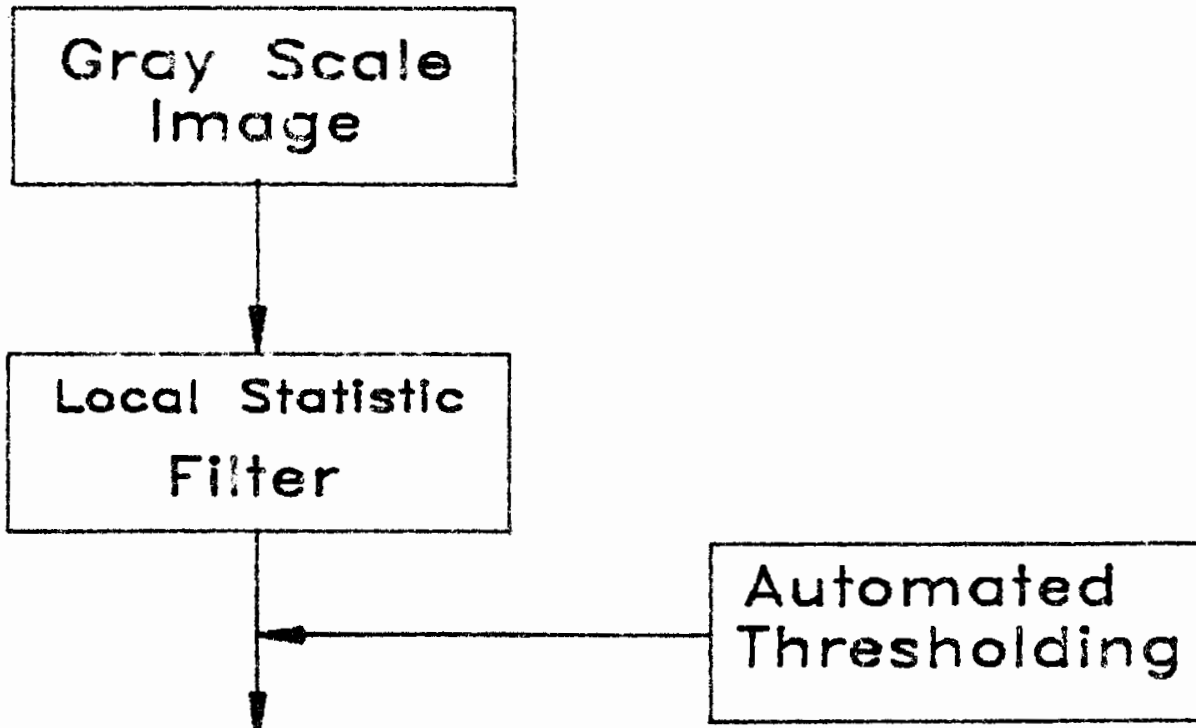

\section{Threshold (a) operation}

\section{Binary Image}

\section{Contour Following}

\section{Binary Contour Image}

Figure 15.Iocal statistic metrod. The gray scale image is processea by the local statistic filter. Based on the filtered gray scale inage, the same operations are performed as in the threshold(a) method. 
Since the local methods variance(n) and Lee's Local Statistic Method take into account a large set of neighboring pixels, these operations should be applied only on those pixels which represent the edge or are close to it. This can be done in two different ways. Both alternatives were implemented and tested.

Option 1: A lower and upper intensity boundary is specified (Figure 16). Each pixel within this range is processed. The intensity range must be selected wide enough so that its boundaries do not influence the decision whether a pixel belongs to an edge or not.

\section{Option 1 - Intensity Specification}

Intensity Histogram

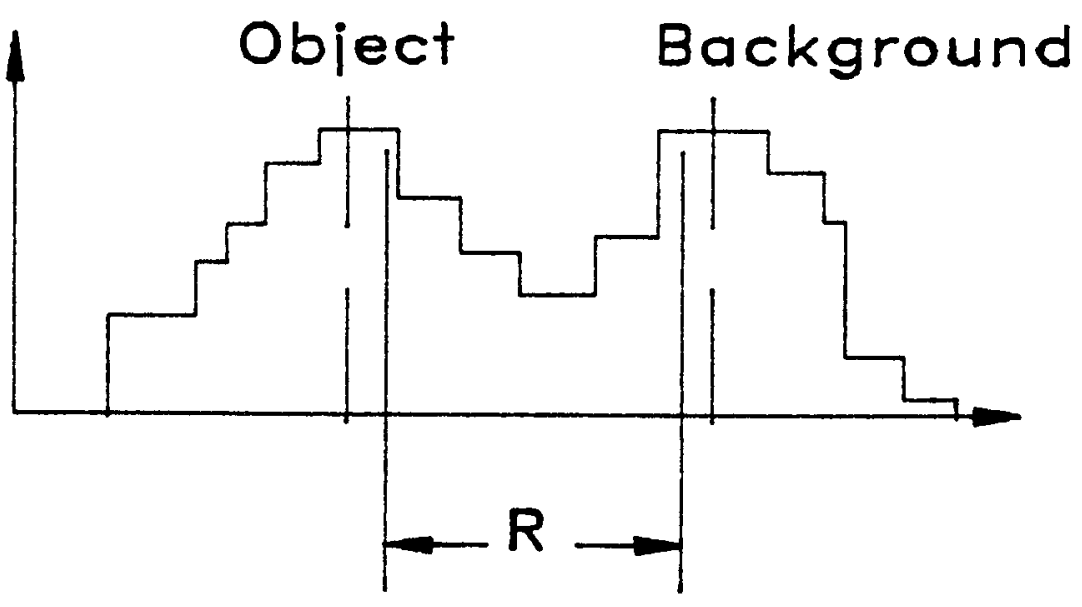

Range of intensity of pixels to be processed

Figure 16. Option 1 of local methods 
Option 2: The second possibility requires a priori knowledge of the approximate edge location (Figure 17). All pixels within a certain band of the approximate edgeline are processed. As in the previous case, this band must be wide enough so that the specification of the estimated location of the edge does not influence the selection of edgepixels.

\section{Option 2 - Local specification}

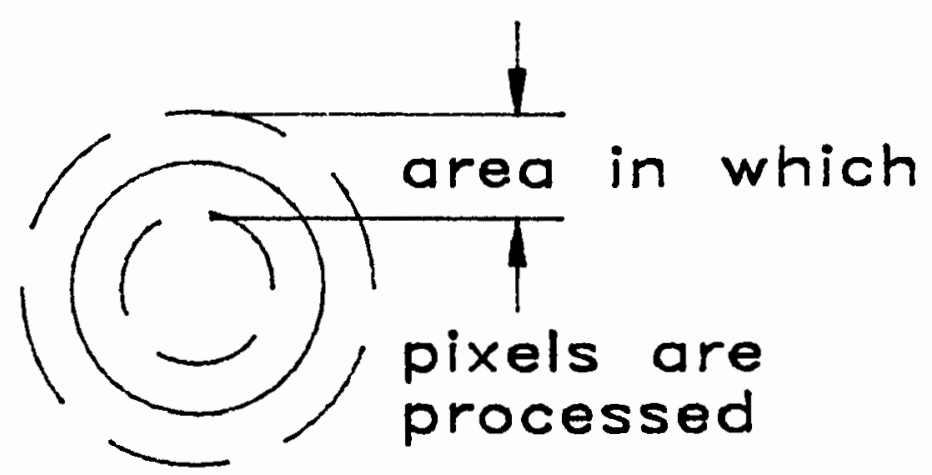

Figure 17.0ption 2 of local methods 
CHAPTER III

COMPARISON OF IMAGE ANALYSIS METHODS

\subsection{MEASURES OF PERFORMANCE}

A comparison of image analysis methods has to be based on objective and meaningful measures of performance. They can be divided into quantitative and qualitative measures.

\subsubsection{Quantitative Measures}

Peli and Malah [9] and also Vliet, et al [10] used Pratt's figure of merit [21] which is the weighted and normalized deviation of an actual edge point from the ideal edge.

In this project, another approach is taken. The performance of the methods is studied with regard to the geometrical parameters of a circle. The deviation of an actual diameter from the ideal diameter is the measure of geometric stability. The number of pixels used to form the circle is the measure of pixel stability and finally the repeatability of a method is the measure of the standard deviation of the diameter of $\mathrm{n}$ detected circles of the same scene. The advantage in using these quantitative measures is that the influence of a specific method on the obtained geometrical parameter, for example, the diameter, is directly revealed.

The above-mentioned quantitative performance measures are defined as: 
1. Geometric stability (GS)

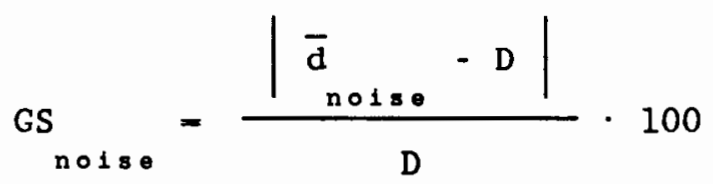

where the subscript "noise" specifies the SNR, $\bar{d}_{n o 1 s \theta}$ is the average diameter obtained of $n$ sample measurements, and $D$ is the diameter detected by the method of an image with no noise. For a perfect image analysis method with regard to geometric stability, GS=0.

\section{Pixe1 Stability (PS)}

The number of edge pixel detected compared to the ideal edge is expressed in the measure of pixel stability as:

$$
\text { PS }-\frac{\text { number of ideal edge points }}{\text { number of detected edge points }}
$$

Pixel stability is the inverted measure of the mean width of an edge [9], which is a known measure in image processing. The inversion is done to obtain a better graphical representation of the results. For a perfect image analysis method with regard to pixel stability, PS=1.

\section{Repeatability of Measurement}

The standard deviation (STD) of the diameter is defined as a measure of repeatability of a method as follows.

$\operatorname{STD}=\left(\frac{\sum(\mathrm{d}-\overline{\mathrm{d}})^{2}}{\mathrm{n}}\right)^{\frac{1}{2}}$ 
where $d$ is the diameter of one of $n$ detected circles and $\bar{d}$ is the average diameter of the $n$ samples. For a perfect image analysis method with regard to repeatability, STD $=0$.

\section{1 .2 Qualitative Measures}

The purpose of the qualitative measures is to give an idea of the appearance of the binary contour image obtained by each method. It is compared to the quantitative measures subjected to the human judgement capability. To assure equal comparison, the following criteria are established.

$$
\begin{aligned}
& \text { Type of contour: CL - closed contour } 1008 \\
& \text { B - broken contour, contour }<808 \\
& \text { CL, B - almost closed, contour }>80 z \text { but } \\
& \text { broken at some points } \\
& \text { Noise occurrence: } \mathbf{N} \text { - noise pixels occur which do not } \\
& \text { belong to the contour }
\end{aligned}
$$

Smoothness of the

$$
\begin{array}{ccc}
\text { circle contour: } & + & - \text { smooth edge } \\
& - & - \text { rough edge } \\
\text { Failure: } & \mathrm{X} & - \text { failure of algorithm }
\end{array}
$$

\subsection{DECISION MODEL}

Using the performance measures, a way must be found to select an optimal method for a certain type of application. For every quantitative and qualitative performance measure, a failure criteria can be defined (Figure 18). The failure of a method is determined by the application and the accuracy demanded by the process of dimensional 


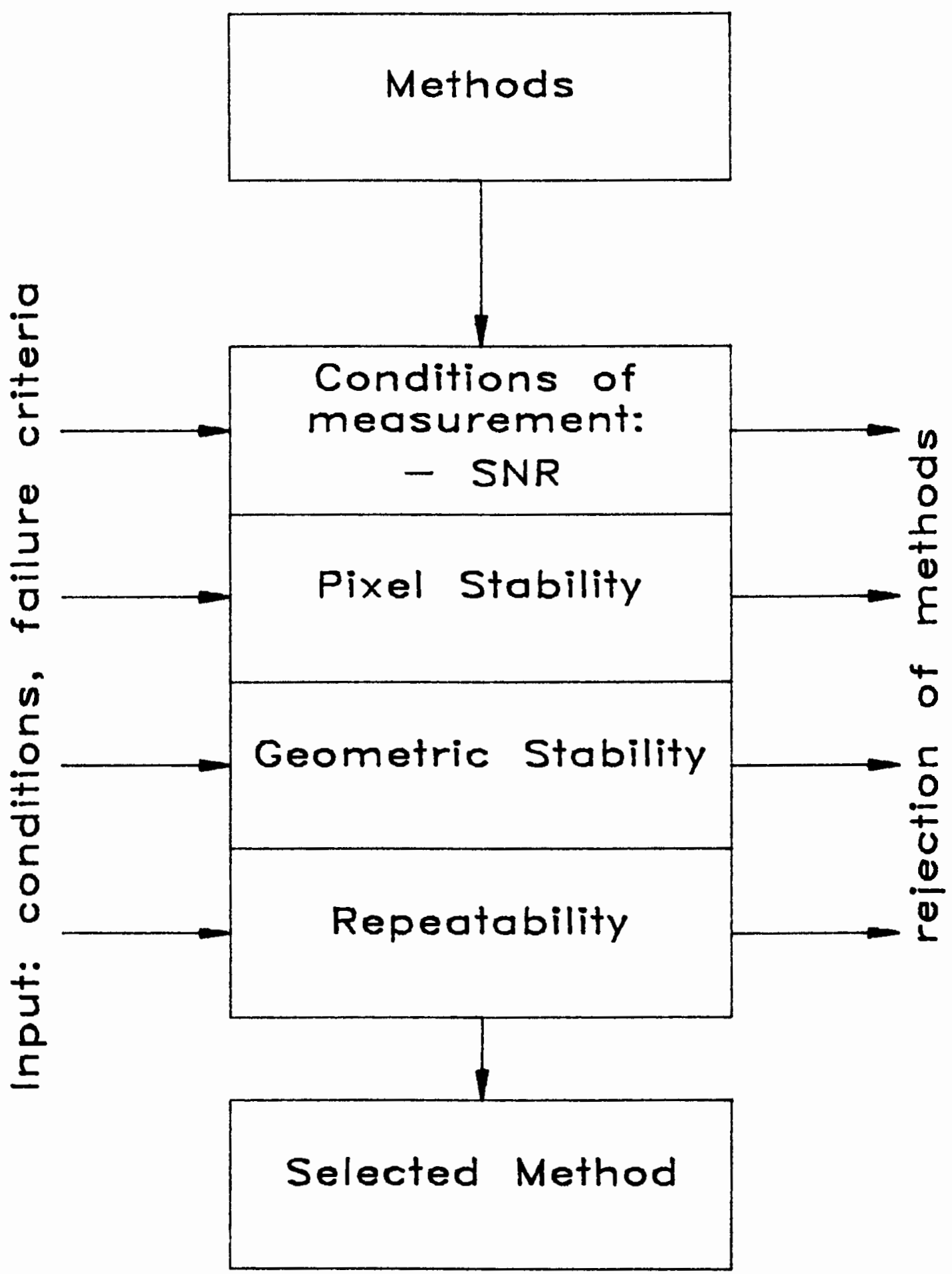

Figure 18,Decision model 
inspection. Using these failure criteria a Decision Model is established. With a certain accuracy required, the failure criteria for pixel, geometric stability, and repeatability can be established. By choosing the measurement conditions, for example, the signal to noise ratio, an additional selection criteria is found. The available set of methods is analyzed whether or not the criteria of the measurement can be satisfied. Rejecting the methods failing this criteria, a subset of methods is selected which can be used for the required task of measurement.

\subsection{IMAGE GENERATOR}

The comparison of these methods is based on images generated by the computer. Generating artificial images rather than using the camera has the advantages that the environment, texture, and geometry of the object and the noise level can be simulated and controlled.

The image generator developed in this project consists of four modules.

Module 1 - Generates a filled circle. Object and background intensity can be specified.

Module 2 - Whereas the first module generates a step edge, the second module smooths the edge and converts it into a ramp edge of width $n$.

Module 3 - Normally distributed random noise with mean $n$ and standard deviation $\mathrm{m}$ is added to the image.

Module 4 - Modeling of texture is done by adding normally distributed noise on object pixels. Later, this 
module could be extended by simulating texture with binary noise or by using other algorithms.

\subsection{SIMULATION MODEL}

The methods were compared on artificially generated images. The cylindrical parts used to study the real edge appearance had a diameter of 160 pixels using typical magnifications. Therefore, the same diameter was selected for the synthetically produced circles. Two contrast levels were chosen: A low contrast simulation of 100 gray values difference (background 140 , object 40 ) and a high contrast simulation of 200 gray values difference (background 240, 40). The low contrast images represent an illumination scene using diffused overhead lighting. The high contrast images simulate lighting typically produced using a diffused glass table where the lights are mounted below.

The simulation was done for ramp edges with a width of 5 pixels. The edge width was determined by studying images of real edges and is similar to other research done using the same edge model [9]. In Figure 19, the modeled edge and the real edge are compared. Gaussian noise with a mean of zero was added to the image. The methods were tested for $\sigma_{n}=0,2,4,8,16$ and 32 for both contrasts. 10 sample images for every simulation case were evaluated. Intensity values exceeding the range of 0 to 255 were clipped. In particular, this had to be done in the high contrast simulation for $\sigma_{n}=16$ and 32 . The results of the methods were evaluated with regard to the Signal to Noise Ratio (SNR) of the synthetical image.

The range of SNR tested in the low contrast simulation is 10 to 


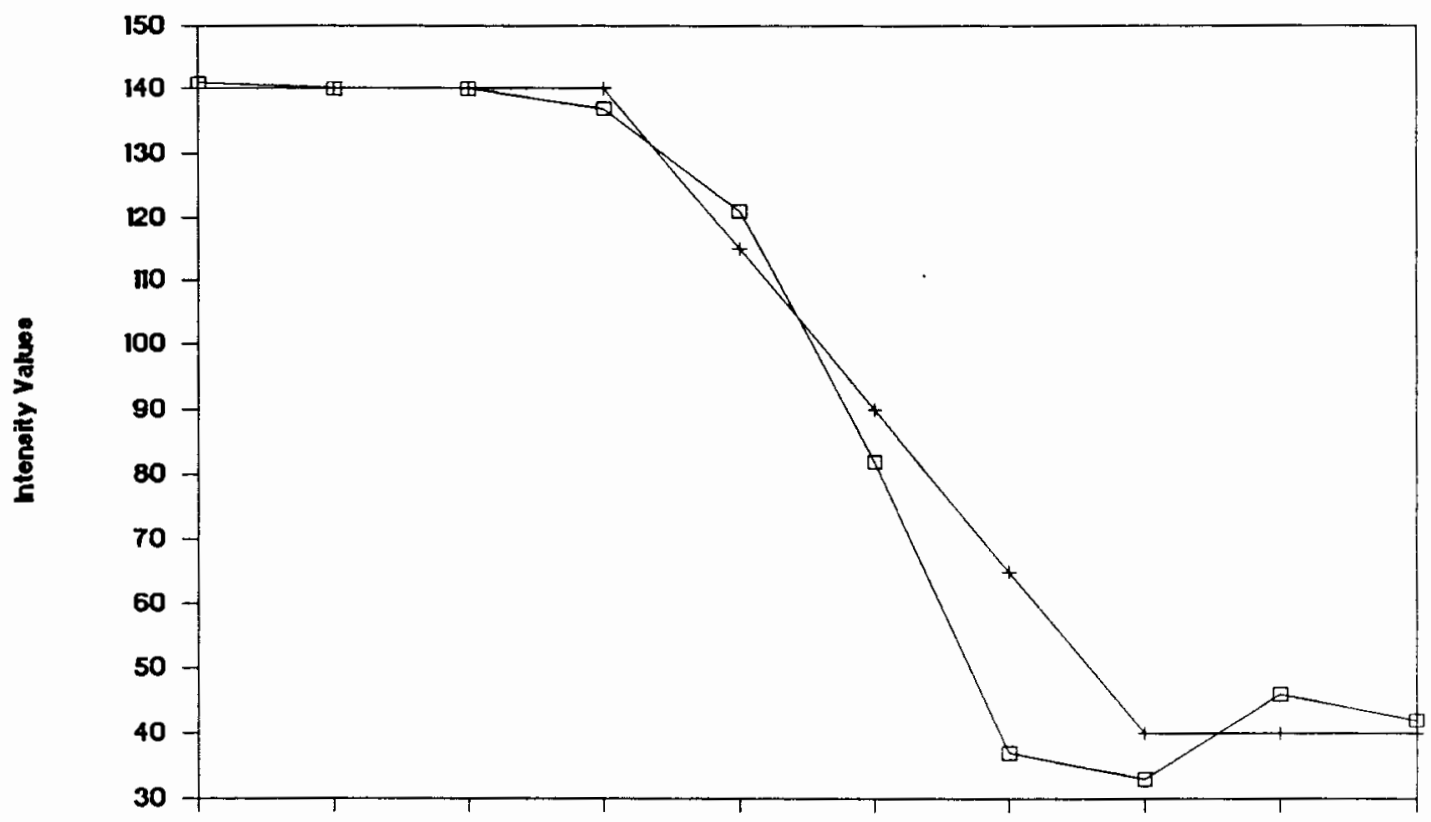

Pixelo

+ Simulated edge $\quad$ Example of real edge Figure 19. Simulation of edge

2500 and for the high contrast it is 40 to 10000 . Although the two ranges overlap, it is advantageous to carry out both simulations, for several reasons. First, the range of SNR is extended by 10 and 10000 . Second, the inaccuracy of the noise simulation due to clipping of intensity values for low SNR is revealed. Third, the redundant data may show the correctness and the weakness of the simulation model.

Every image analysis method demands a careful selection of the input parameters, for example, lowcut and highcut or the range specification on intensity for the pixels to be processed. The process of dimensional inspection requires a complete knowledge of the edge. Therefore the experiment input parameters for the methods were chosen so that for a minimum amount of computation time for each method, a closed 
contour of the edge could be detected. This was done for the ideal image without noise $(\operatorname{SNR}=\infty)$. The input parameters for each method and the simulation case are documented in Appendix $G$. To have meaningful comparison of the methods, the input parameters were not readjusted for increasing noise. In addition, the experiments made during this project using these methods, showed that a readjustment of input parameters does not improve the evaluation significantly enough to take it into consideration.

\subsection{SIMULATION TEST RESULTS}

For the low and the high contrast simulations, the results are documented in a set of tables and figures. To familiarize the reader with the graphs, a short general description is given below for each type of table and figure. The results are analyzed and discussed in the next section.

Tables III and IV show the quantitative results of the simulation. For each method and different signal to noise ratios, the pixel stability, geometric stability and the repeatability are documented. Using data of the Tables III and IV, graphs of the pixel stability and the geometric stability versus the signal to noise ratio were generated. For each category two sets of graphs were drawn. The first set compares all methods besides the methods using evaluation option 2 . The second set compares the results of the methods using options 1 and 2 .

Figures $20,21,24$, and 25 show the pixel stability versus the Signal to Noise Ratio graphs. The horizontal line for pixel stability (PS)=1 would represent a perfect image analysis method with respect to 
the number of pixels used. PS $>1$ means that the image analysis methods detected less edge points than the number of Ideal edge points and vice versa.

Figures $22,23,26$, and 27 show the geometric stability (GS) versus the signal to noise ratio graphs. The scale of the geometric stability is logarithmic. All methods with GS exceeding a given boundary value fail.

Tables $V$ and VI show the qualitative results. 
TABLE III

QUALITATIVE TEST RESULTS OF LOW CONTRAST SIMULATION

PIXEL STABILITY

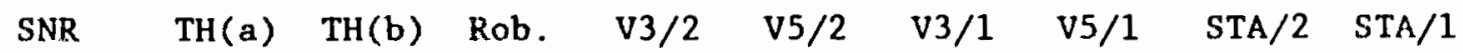

\begin{tabular}{rlllllllll}
\hline 10 & - & 0.115 & - & 0.344 & 0.352 & - & - & 0.850 & 0.810 \\
40 & 0.835 & 0.539 & 0.107 & 0.653 & 0.727 & 0.558 & 0.940 & 0.932 & 0.932 \\
156 & 0.895 & 0.953 & 0.564 & 1.012 & 0.956 & 1.144 & 0.999 & 0.983 & 0.983 \\
625 & 0.892 & 0.998 & 1.335 & 1.141 & 1.038 & 1.161 & 1.038 & 0.996 & 0.996 \\
2500 & 0.900 & 1 & 1.300 & 1.110 & 1.053 & 1.110 & 1.053 & 0.998 & 0.998 \\
$;$ & 1 & 1 & 1 & 1 & 1 & 1 & 1 & 1 & 1
\end{tabular}

GEOMETRIC STABILITY

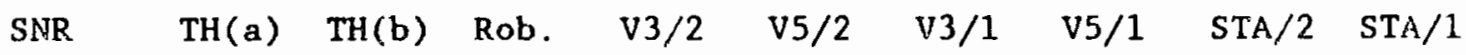

\begin{tabular}{rlllllllll}
\hline 10 & - & 2.551 & - & 0.389 & 0.014 & - & - & 0.038 & 0.046 \\
40 & 0.666 & 1.896 & 1.900 & 0.039 & 0.001 & 1.712 & 0.008 & 0.001 & 0.003 \\
156 & 0.598 & 0.016 & 1.284 & 0.038 & 0.015 & 0.041 & 0.012 & 0.007 & 0.007 \\
625 & 0.552 & 0.002 & 0.011 & 0.007 & 0.004 & 0.009 & 0.004 & 0.007 & 0.011 \\
2500 & 0.441 & 0 & 0.054 & 0.007 & 0.001 & 0.007 & 0.001 & 0.001 & 0.001 \\
$;$ & 0 & 0 & 0 & 0 & 0 & 0 & 0 & 0 & 0
\end{tabular}

REPEATABILITY OF MEASUREMENT

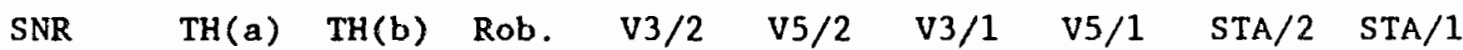

\begin{tabular}{rlllllllll}
\hline 10 & - & 0.428 & - & 0.576 & 0.097 & - & - & 0.099 & 0.090 \\
40 & 0.064 & 1.050 & 0.279 & 0.086 & 0.035 & 0.749 & 0.033 & 0.065 & 0.063 \\
156 & 0.050 & 0.021 & 1.021 & 0.046 & 0.030 & 0.038 & 0.034 & 0.042 & 0.042 \\
625 & 0.046 & 0.004 & 0.048 & 0.035 & 0.021 & 0.032 & 0.021 & 0.035 & 0.035 \\
2500 & 0.026 & 0 & 0.198 & 0.016 & 0.012 & 0.017 & 0.012 & 0.034 & 0.034 \\
$;$ & 0 & 0 & 0 & 0 & 0 & 0 & 0 & 0 & 0
\end{tabular}


TABLE IV

QUANTITATIVE TEST RESULTS OF HIGH CONTRAST SIMULATION

PIXEL STABILITY

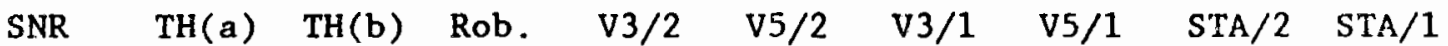

\begin{tabular}{rlllllllll}
\hline 40 & 0.837 & 0.595 & 0.174 & 0.841 & 0.871 & 1.052 & 0.929 & 0.961 & 0.960 \\
156 & 0.884 & 0.986 & 0.791 & 1.026 & 0.780 & 1.153 & 0.905 & 0.987 & 0.987 \\
625 & 0.891 & 0.999 & 1.368 & 1.091 & 0.927 & 1.112 & 0.927 & 0.995 & 0.995 \\
2500 & 0.893 & 1 & 1.341 & 1.070 & 0.971 & 1.071 & 0.971 & 0.999 & 0.999 \\
10000 & 0.910 & 1 & 1.296 & 1.038 & 1.007 & 1.038 & 1.007 & 1 & 1 \\
$;$ & 1 & 1 & 1 & 1 & 1 & 1 & 1 & 1 & 1
\end{tabular}

GEOMETRIC STABILITY

SNR TH(a) TH(b) Rob. V3/2 V5/2 V3/1 V5/1 $\quad$ STA/2 $\quad$ STA $/ 1$

\begin{tabular}{rlllllllll}
\hline 40 & 0.687 & 1.260 & 6.102 & 0.234 & 0.226 & 2.372 & 0.099 & 0.100 & 0.100 \\
156 & 0.638 & 0.008 & 7.305 & 0.042 & 0.105 & 0.009 & 0.096 & 0.032 & 0.032 \\
625 & 0.630 & 0 & 0.008 & 0.014 & 0.030 & 0.010 & 0.030 & 0.002 & 0.002 \\
2500 & 0.568 & 0 & 0.001 & 0.001 & 0.024 & 0.001 & 0.024 & 0.004 & 0.004 \\
10000 & 0.437 & 0 & 0.001 & 0.004 & 0.005 & 0.004 & 0.005 & 0.009 & 0.009 \\
$;$ & 0 & 0 & 0 & 0 & 0 & 0 & 0 & 0 & 0
\end{tabular}

REPEATABILITY OF MEASUREMENT

SNk TH(a) TH(b) Rob. Vs/2 V5/2. V3/1 v5/.. STA/2 STA 1

\begin{tabular}{rlllllllll}
\hline 40 & 0.058 & 1.102 & 0.599 & 0.018 & 0.058 & 0.673 & 0.058 & 0.068 & 0.066 \\
156 & 0.035 & 0.039 & 0.558 & 0.044 & 0.035 & 0.040 & 0.033 & 0.053 & 0.053 \\
625 & 0.041 & 0.003 & 0.015 & 0.032 & 0.032 & 0.023 & 0.032 & 0.045 & 0.045 \\
2500 & 0.162 & 0 & 0.040 & 0.018 & 0.036 & 0.017 & 0.036 & 0.038 & 0.038 \\
10000 & 0.041 & 0 & 0.034 & 0.015 & 0.009 & 0.015 & 0.010 & 0.033 & 0.033 \\
$;$ & 0 & 0 & 0 & 0 & 0 & 0 & 0 & 0 & 0
\end{tabular}

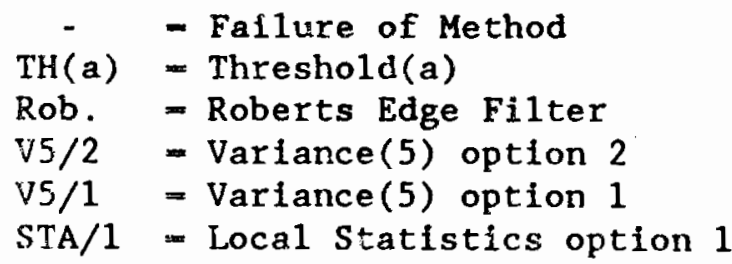

SNR - Signal to Noise Ratio

TH(b) - Threshold(b)

V3/2 - Variance (3) option 2

V3/1 - Variance(3) option 1

$\mathrm{STA} / 2=$ Local Statistics option 2. 


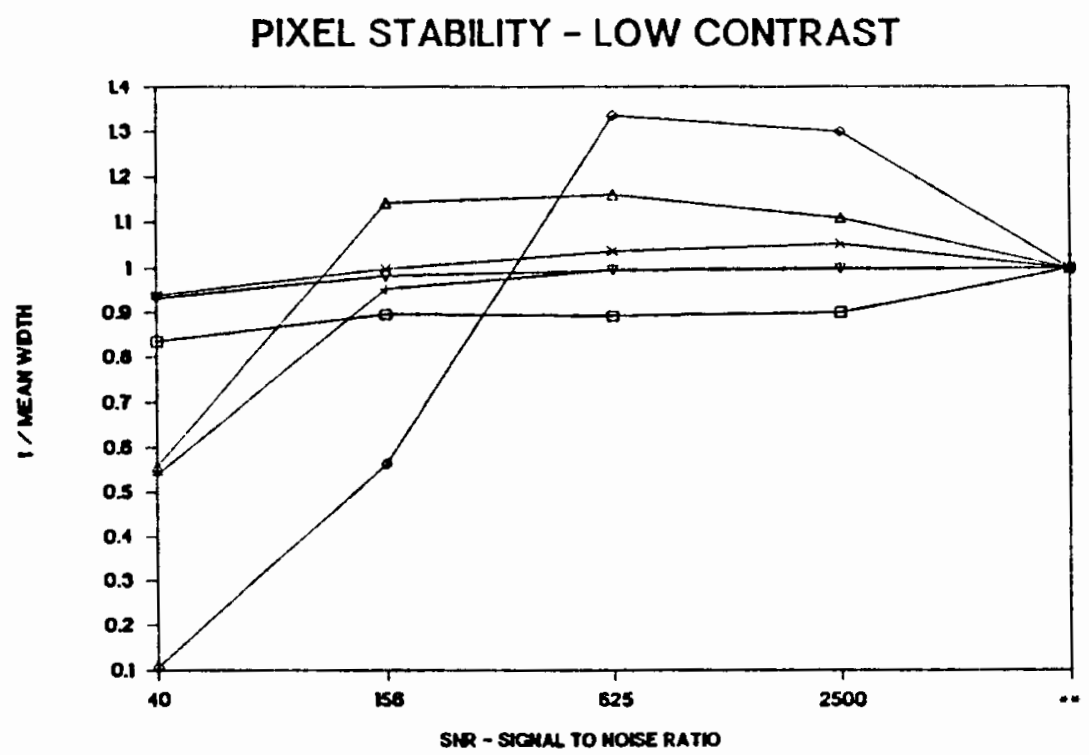

a Threshold(a)

- Roberts Edge Filter

$\mathrm{x}$ Variance(5) option 1

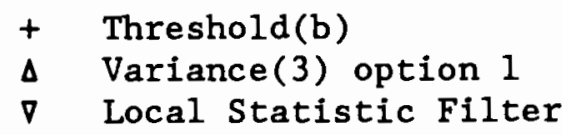

Figure 20,Pixel stability - Iow contrast 1

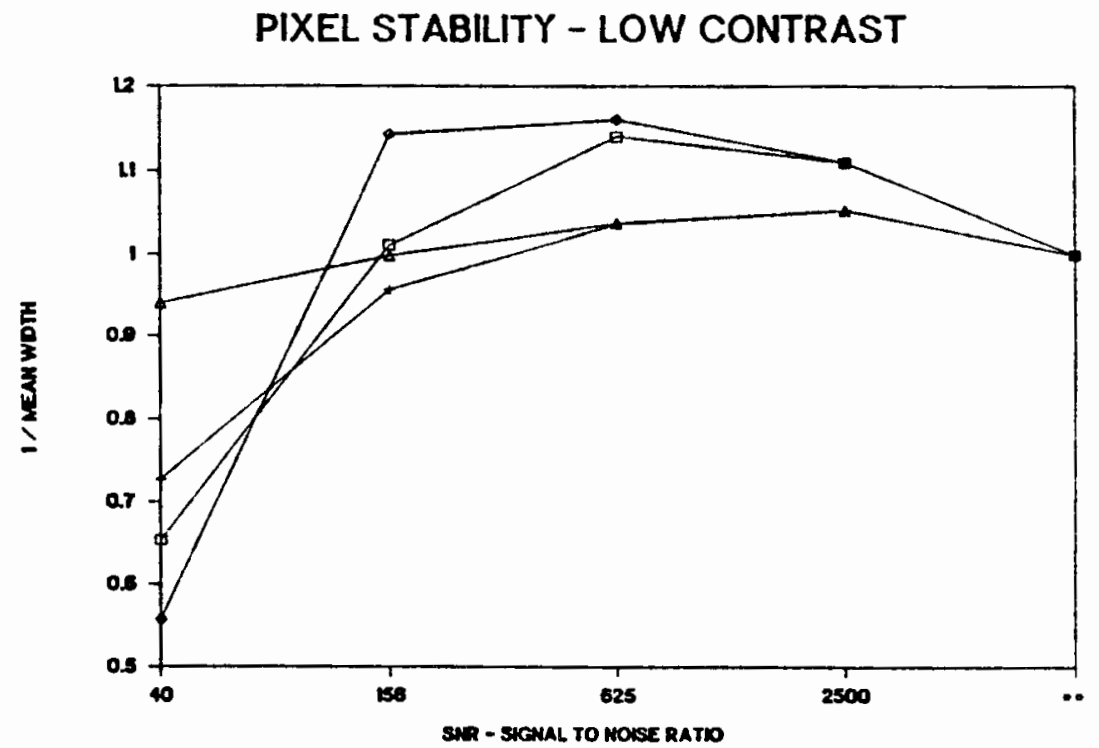

- Variance(3) option 1

$\Delta \quad$ Variance (5) option 1

a Variance(3) option 2

+ Variance(5) option 2 
Geometric Stability vs. SNR

Low contrast

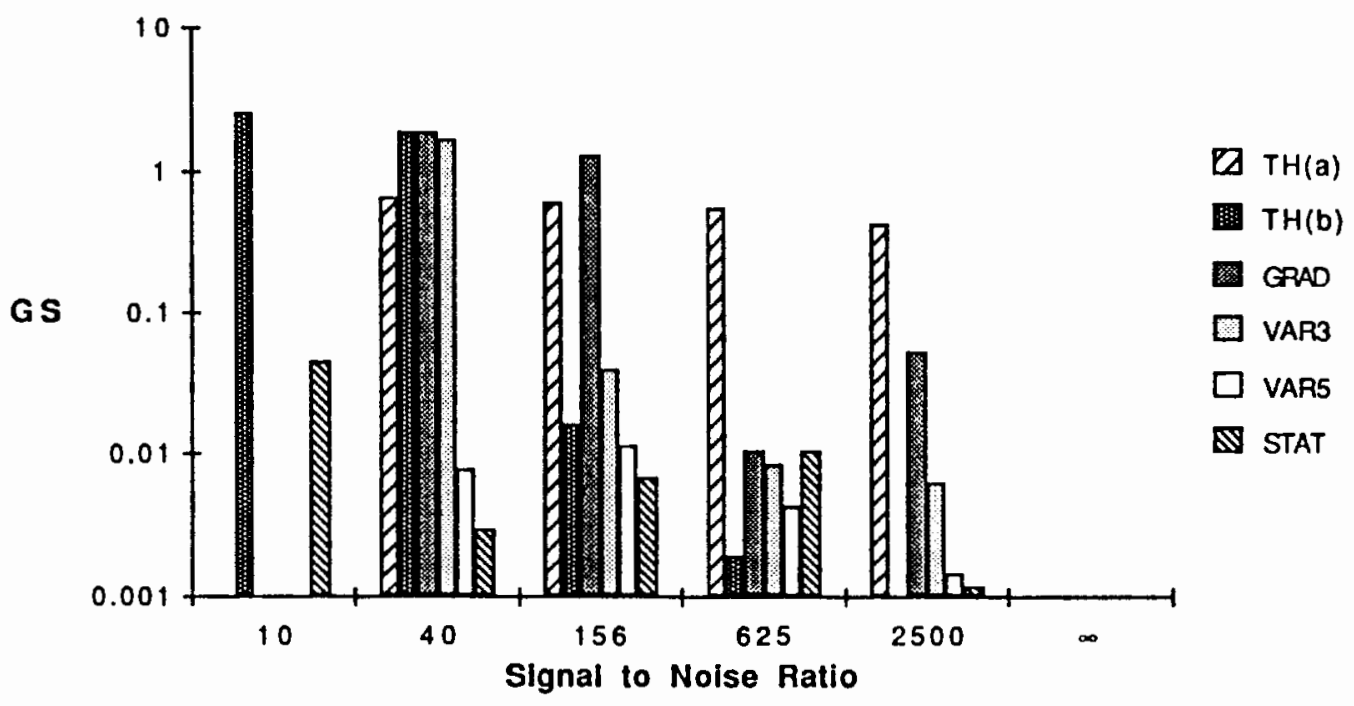

Figure 22,Geometric stability - low contrast 1

Geometric Stability vs. SNR

Low contrast

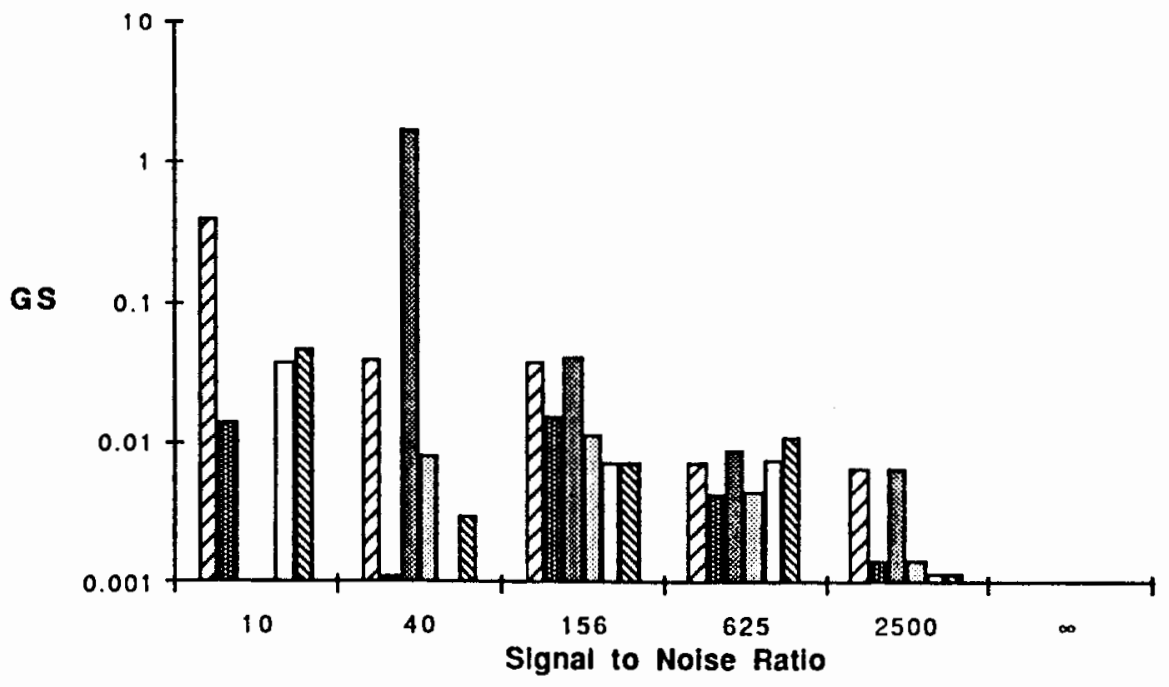

$\square$ VAR3 LOC

四 VAR5 LOC

VAR3

VAR5

$\square$ STAT LOC

STAT

Figure 23, Geometric stability - low contrast 2 


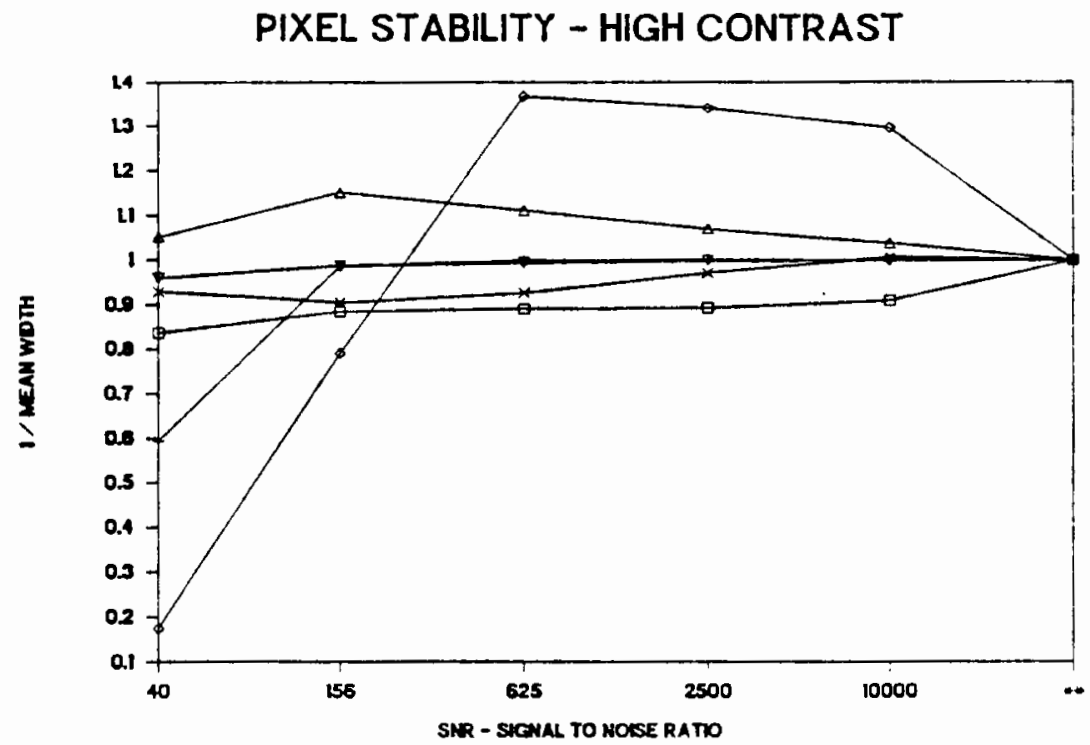

a Threshold(a)

$\checkmark$ Roberts Edge Filter

+ Threshold(b)

$\mathrm{x}$ Variance(5) option 1

$\Delta$ Variance(3) option 1

$\nabla$ Local Statistic Filter

Figure 24.Pixe1 stability - high contrast 1

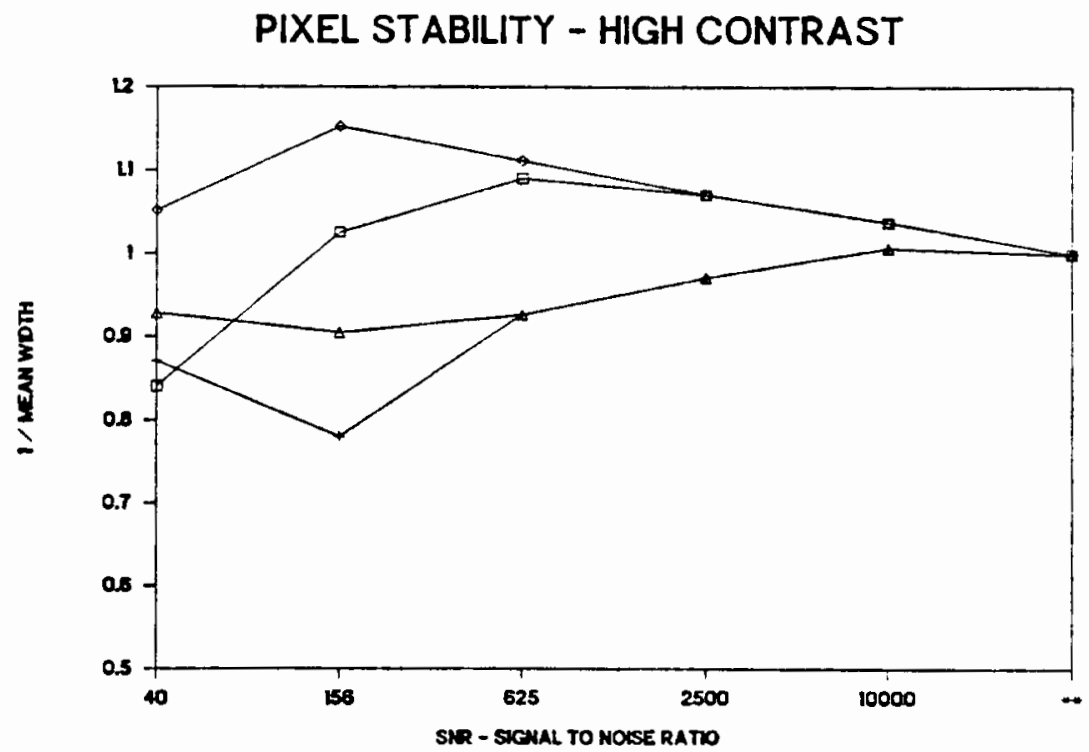

$\checkmark \quad$ Variance(3) option 1

$\Delta \quad$ Variance(5) option 1

- Variance (3) option 2

+ Variance(5) option 2 
Geometric Stability vs. SNR

High contrast

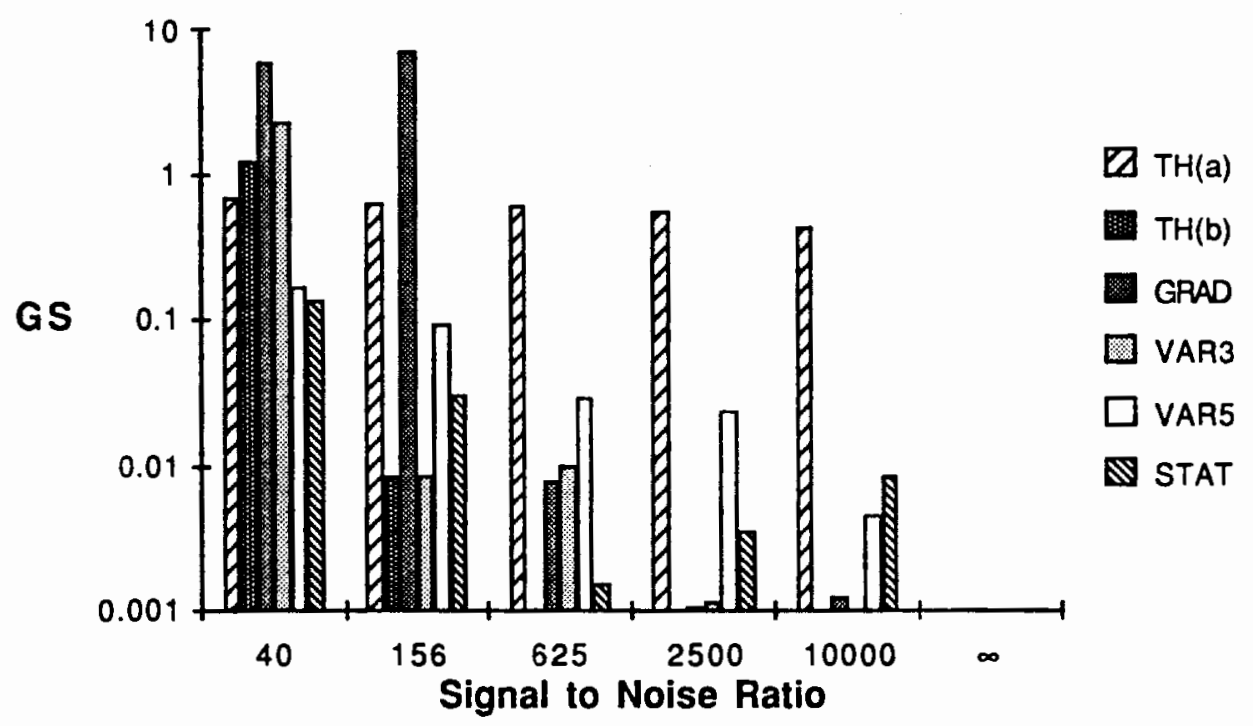

Figure 26. Geometric stability - high contrast 1

Geometric Stability vs. SNR

High contrast

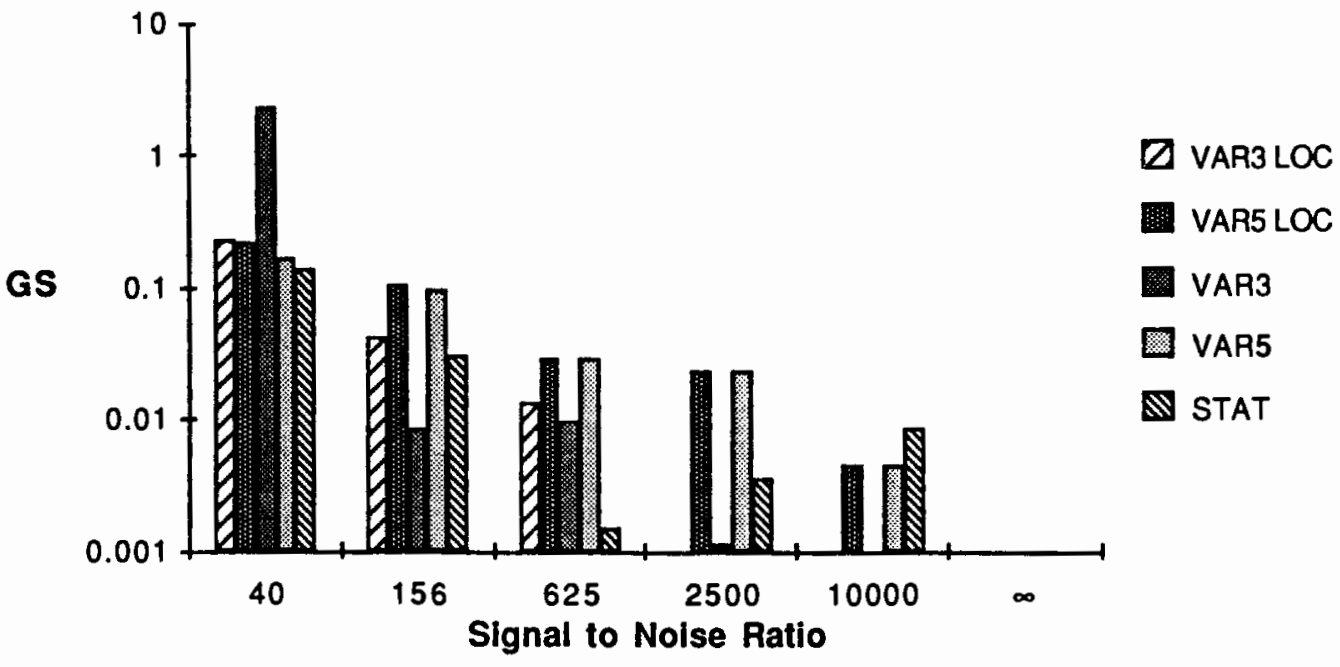

Figure 27.Geometric stability - high contrast 2 
Qualitative Test Results

TABLE V

QUALITATIVE TEST RESULTS OF LOW CONTRAST SIMULATION

\begin{tabular}{lclllll} 
SNR & 10 & 40 & 156 & 625 & 2500 & $\infty$ \\
\hline threshold(a) & $\mathrm{X}$ & CL- & CL- & CL & CL & CL+ \\
threshold(b) & $\mathrm{X}, \mathrm{N}$ & $\mathrm{X}, \mathrm{N}$ & $\mathrm{CL}, \mathrm{B}$ & $\mathrm{CL}+, \mathrm{B}$ & $\mathrm{CL}+$ & $\mathrm{CL}+$ \\
Roberts edge filter & $\mathrm{X}$ & $\mathrm{X}, \mathrm{N}$ & $\mathrm{X}, \mathrm{N}$ & $\mathrm{CL}$ & $\mathrm{CL}$ & $\mathrm{CL}$ \\
Variance(3) & $\mathrm{X}$ & $\mathrm{X}, \mathrm{N}$ & CL- & CL- & CL- & CL \\
Variance(5) & $\mathrm{X}$ & CL- & CL- & CL & CL+ & CL+ \\
Variance(3) opt2. & $\mathrm{X}, \mathrm{CL}-$ & $\mathrm{X}, \mathrm{CL}-$ & CL- & CL- & CL- & CL \\
Variance(5) opt2. & $\mathrm{X}, \mathrm{CL}-$ & $\mathrm{X}, \mathrm{CL}-$ & $\mathrm{CL}$ & $\mathrm{CL}$ & $\mathrm{CL}+$ & CL+ \\
Local Statistic & CL- & CL- & CL & CL & CL+ & CL+ \\
Local Statistic opt2. & CL- & CL- & CL & CL & CL+ & CL+
\end{tabular}

TABLE VI

QUALITATIVE TEST RESULTS OF HIGH CONTRAST SIMULATION

\begin{tabular}{lllllll} 
SNR & 40 & 156 & 625 & 2500 & 10000 & $\infty$ \\
\hline threshold(a) & CL- & CL- & CL- & CL- & CL+ & CL+ \\
threshold(b) & X, B & CL, B & CL+, B & CL+, B & CL+, B & CL+ \\
Roberts edge filter & X,N & CL, N & CL & CL & CL & CL+ \\
Variance(3) & X, CL & CL- & CL- & CL & CL & CL \\
Variance(5) & CL & CL & CL & CL+ & CL+ & CL+ \\
Variance(3) opt2. & X, CL- & CL- & CL- & CL & CL & CL \\
Variance(5) opt2. & X, CL & CL & CL & CL+ & CL+ & CL+ \\
Local Statistic & CL- & CL & CL & CL & CL+ & CL+ \\
Local Statistic opt2. & CL- & CL & CL & CL & CL+ & CL+
\end{tabular}

For each method, the simulation results for different Signal to Noise Ratios are discussed, and the problems of applying a method to synthetical images are pointed out. The discussion on the result analysis does not refer to any specific figures and tables. It is up to the reader to follow the analysis in the above given graphs. A 
statement of comparison is given after the discussion of the results of the real image experiments where advantages and disadvantages become more obvious.

In the discussion below, the judgement whether a method succeeds or fails is based on the following approximated criteria:

Pixel stability fails when $\quad$ PS $>0.8$

Geometric stability fails when GS $>0.1$

Repeatability fails when STD $>0.1$

\subsubsection{Threshold(a) method}

Because of the fact that the edgepixels are found by contour following, the measure of pixel stability is consistent over the entire range of SNR tested. The step in the measure of geometric stability between the image of $S N R=\infty$ and the images with noise is due to the fact that the threshold value is chosen to be half the intensity value between object and background intensity. This of course interacts with the model of the edge, where the pixel intensity of the middle of the edge corresponds with the chosen threshold. The increase of noise in the image will be detected immediately. This is a problem of simulation. For low SNR the edge of the circles becomes rougher. In the low contrast case for $S N R=10$, no closed contour is observed and therefore the contour following algorithm fails.

\subsubsection{Threshold(b) method}

By studying the quantitative performance measures, thresholding(b) performs well for the range $S N R>150$. But the qualitative measures show that for SNR $<2500$ the edge contour is broken, but on the other hand, 
the edge is smooth and close to the smoothness of an edge of a perfect circle. For $S N R<=40$, the failure of this method is observed due to occurrence of noise pixels.

\subsubsection{Roberts Edge Filter method}

The ideal image of the contour without noise consists of a triple edge. A closed single contour edge cannot be obtained due to the characteristics of Roberts edge filter (see also [9]). For $625<\mathrm{SNR}<$ 10000 , less pixels are used to build the edge which results in pixel stability $>1$. Pixel and geometric stability shows failure for $\mathrm{SNR}<-156$, in the low contrast case. In the high contrast case, the measure of pixel stability shows failure for $S N R<=40$, but the measure of geometric stability and repeatability reveal that this method fails for even $S N R<-156$. In this case pixels which do not belong to the edge exceed the gradient threshold, but not significantly enough for the pixel stability measure to show failure. This is one example where it is demonstrated, that the measure of pixel stability does not imply geometric stability.

\section{$\underline{3.5 .4 \text { Variance }(n) \text { method }}$}

Compared to variance(3), variance(5) detects a smoother edge. Variance(3) performs well for $156<$ SNR $<\infty$ for both options. By using variance(5) option 1 extends the range of use to $40<\mathrm{SNR}<\infty$. Option 2 instead, only performs as well as variance(3). It can be seen that for low SNR, better results are obtained by specifying the intensity range, where the pixels are processed (option 1) combined with a larger neighborhood size of pixels to smooth the noise pixels. The larger the 
$\mathrm{n} \times \mathrm{n}$ variance window, the more features can be enhanced properly from noisy images. For option 2 and $S N R=10$ in the low contrast case it is demonstrated, that geometric stability does not imply pixel stability. In this case the specification of the approximate knowledge of the edge influences the edge pixel enhancement. Every pixel in the specified area is detected as an edge pixel, therefore this method seems to be geometrically stable for SNR-10.

\section{5 .5 Local Statistic method}

Lee's Local method differs from thresholding(a) in that a preprocessing of the image is done. The result of the simulation show that this method performs well over the entire range. It is the best method with respect to geometric stability. The computation time to process an image is higher compared to variance(3) and variance(5). For SNR $<-40$ the detected edge is rough, but still the radius estimation algorithm gives repeatable results. Within the tested range there is no significant difference between option 1 and 2 observed.

\subsection{REAL IMAGE EXPERIMENTS}

The different performances of the methods, become more obvious by applying them to real world images with low Signal to Noise ratio acquired by the CCD camera. For this purpose images of a brass cylinder with typical metal texture surface and a wood block with texture lines on a white background were processed. The input parameters for the methods were found by trying to obtain a closed contour without occurence of noise pixels. For every method the qualitative results in means of images are shown in Figures 28-41. The pictures are 
photographs taken from a black and white display monitor. Unfortunately, the quality of these pictures is not very high. The stripes are produced by the display monitor and are not part of the images.

\subsubsection{Circular Objects}

The original gray scale image of the brass cylinder with textured surface is shown in Figure 28. The scene is illuminated by diffuse overhead lighting. The Signal to Noise ratio of this image is determined to SNR=156. In the lower part of the image the threshold(a) method (Figure 29) fails. The circle is flattened. This occurs due to the shadow zone and the low contrast, which can be seen in the original gray scale image. By comparing the contour image extracted by the local statistic method (Figure 30 ), with the result of the threshold(a) method, the advantage of the local statistic method is observed. The shape of the circle is more acceptable than that detected by the threshold(a) method. Still in the lower left corner, local statistic fails, and noise is introduced in the contour. The contrast of the texture of the cylinder is higher than the contrast of the edge in the lower left and right part of the image. Therefore the variance threshold for the variance(5) method (Figure 31) can either be chosen to reject noise pixels and fail to detect a close contour or vice versa. The same problem occurs using the Roberts edge filter (Figure 32). Compared to variance(5), the algorithm is more sensitive to contrast variations and therefore more noise pixels are detected. The threshold(b) method fails as well (Figure 33). The intensity range of the edge pixels cannot be specified accurate enough to detect the edge 
properly. Noise pixels occur in the background as well as in the object.

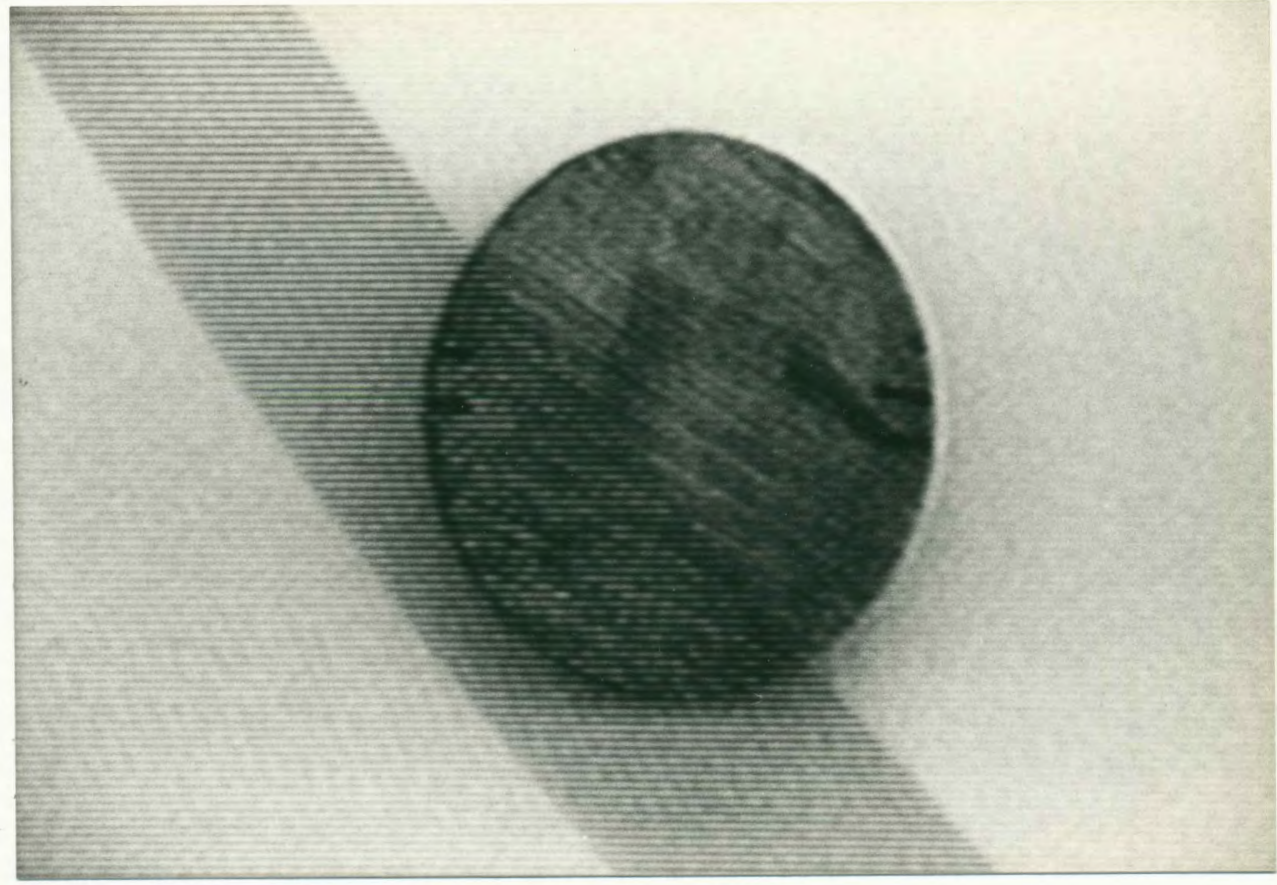

Figure 28. Gray scale of image of brass cylinder with textured surface 


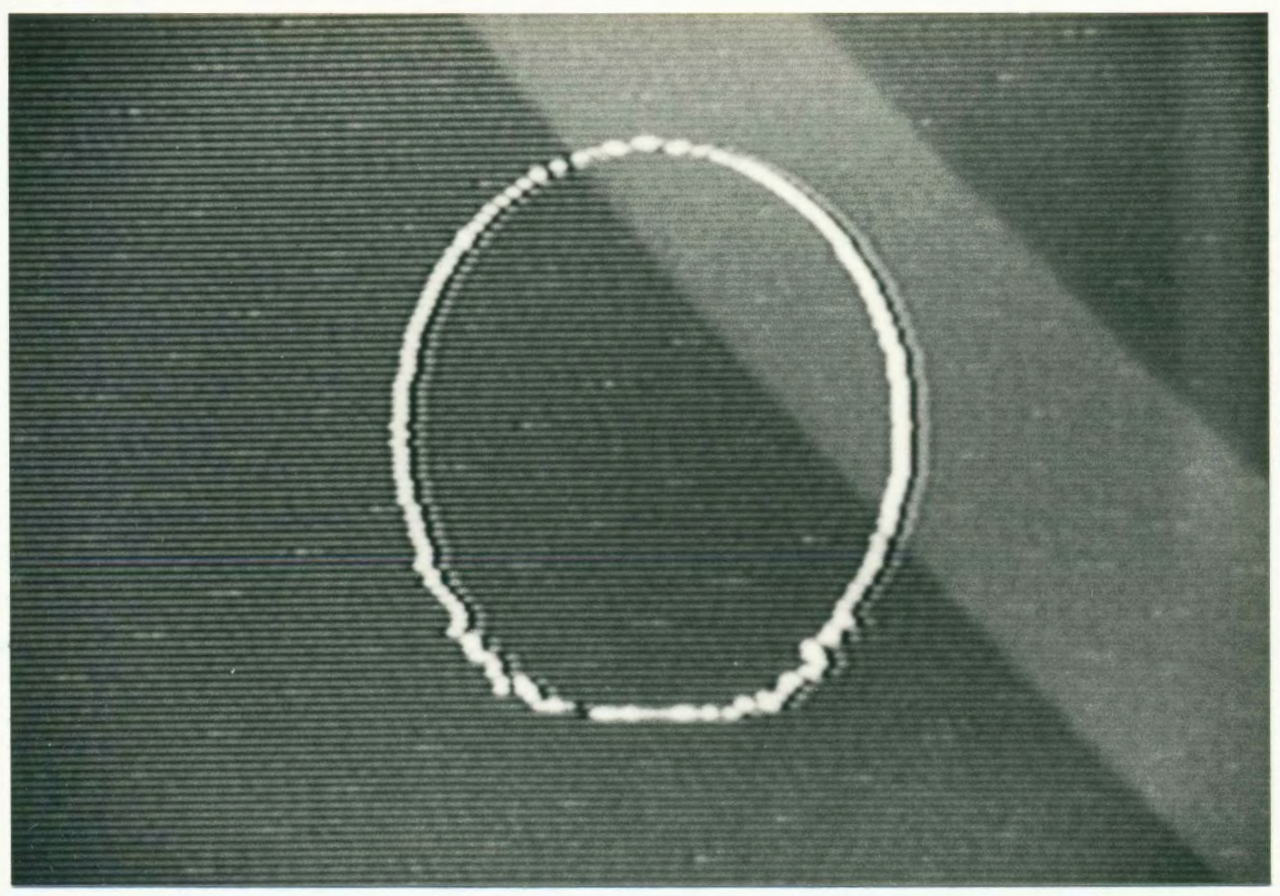

Figure 29. Contour image extracted by the threshold(a) method

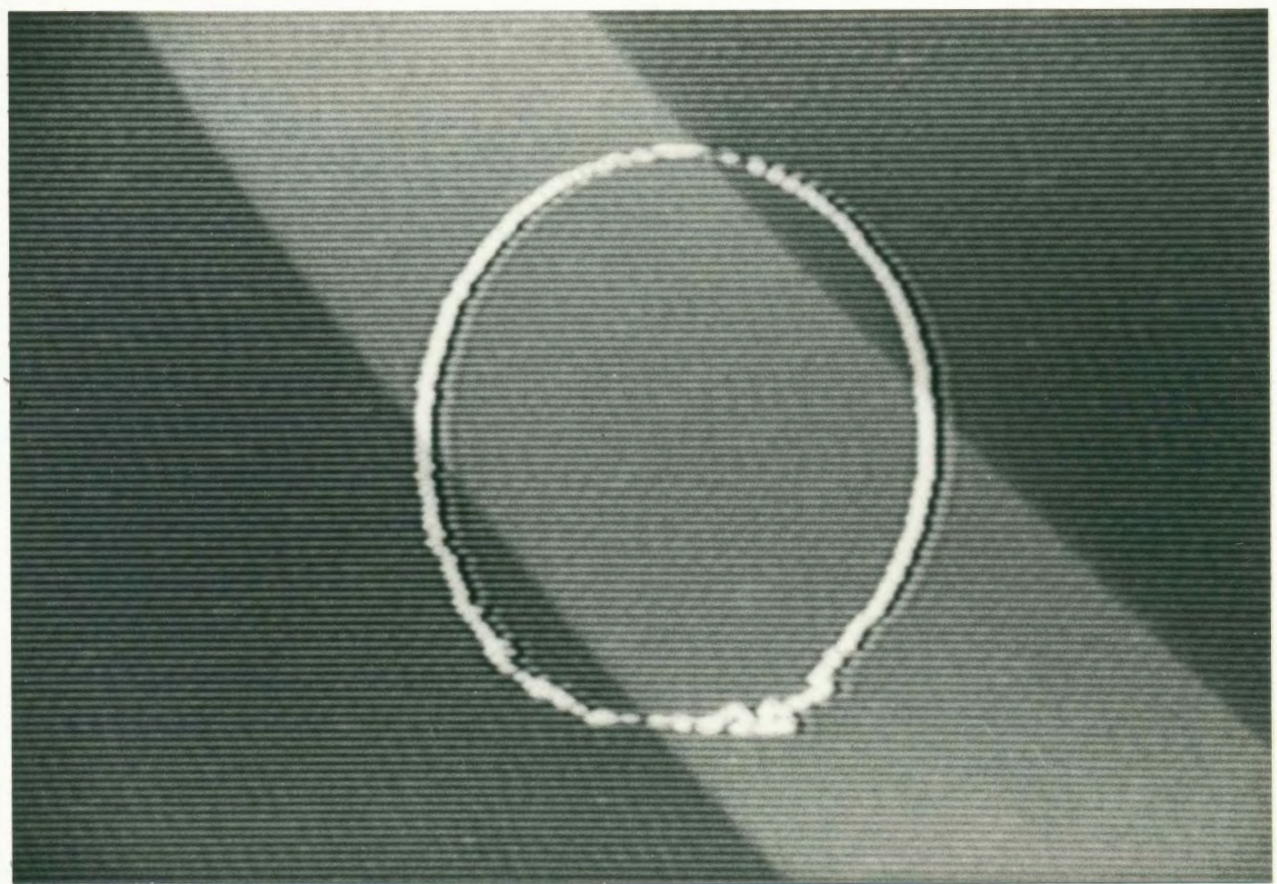

Figure 30 . Contour image extracted by the local statistic method 


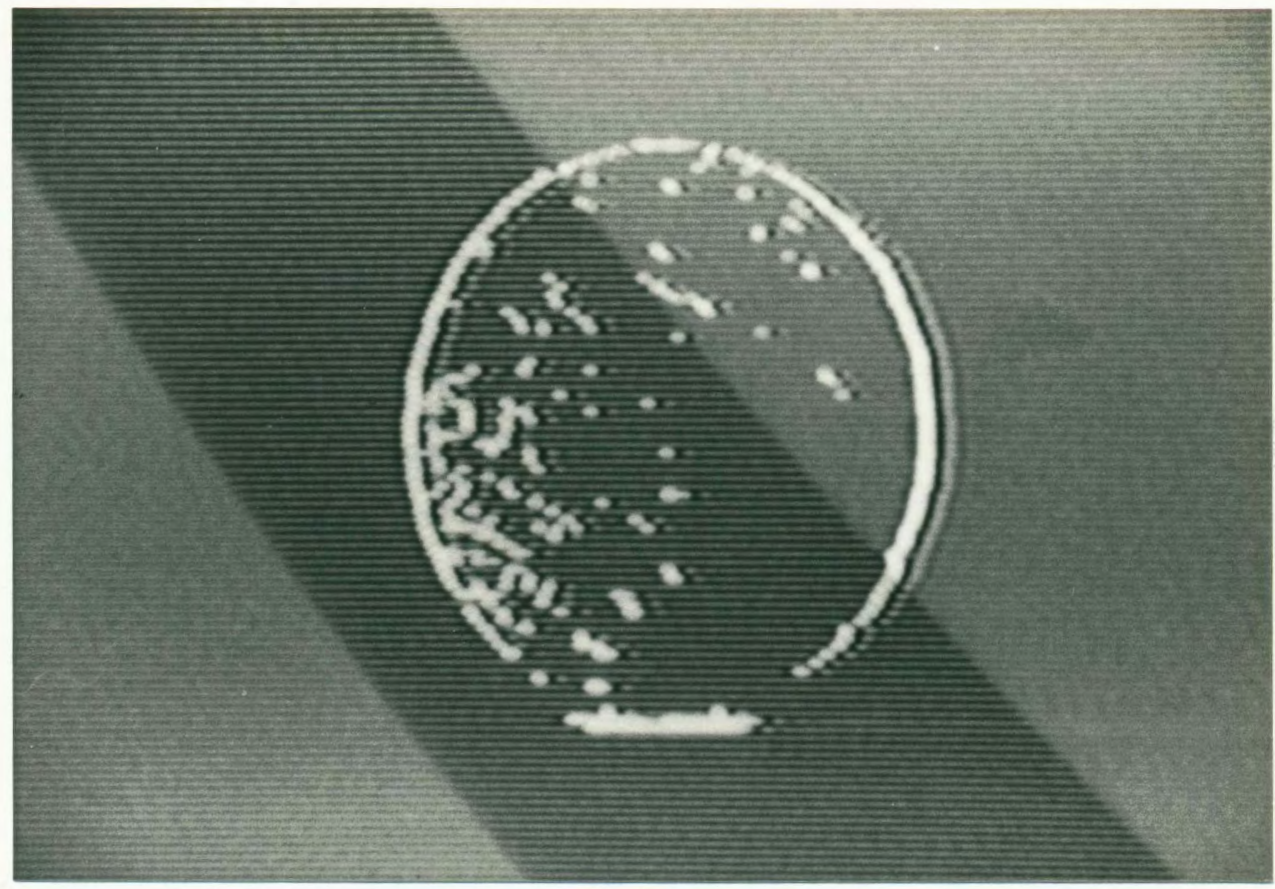

Figure 31. Contour image extracted by the variance(5) method

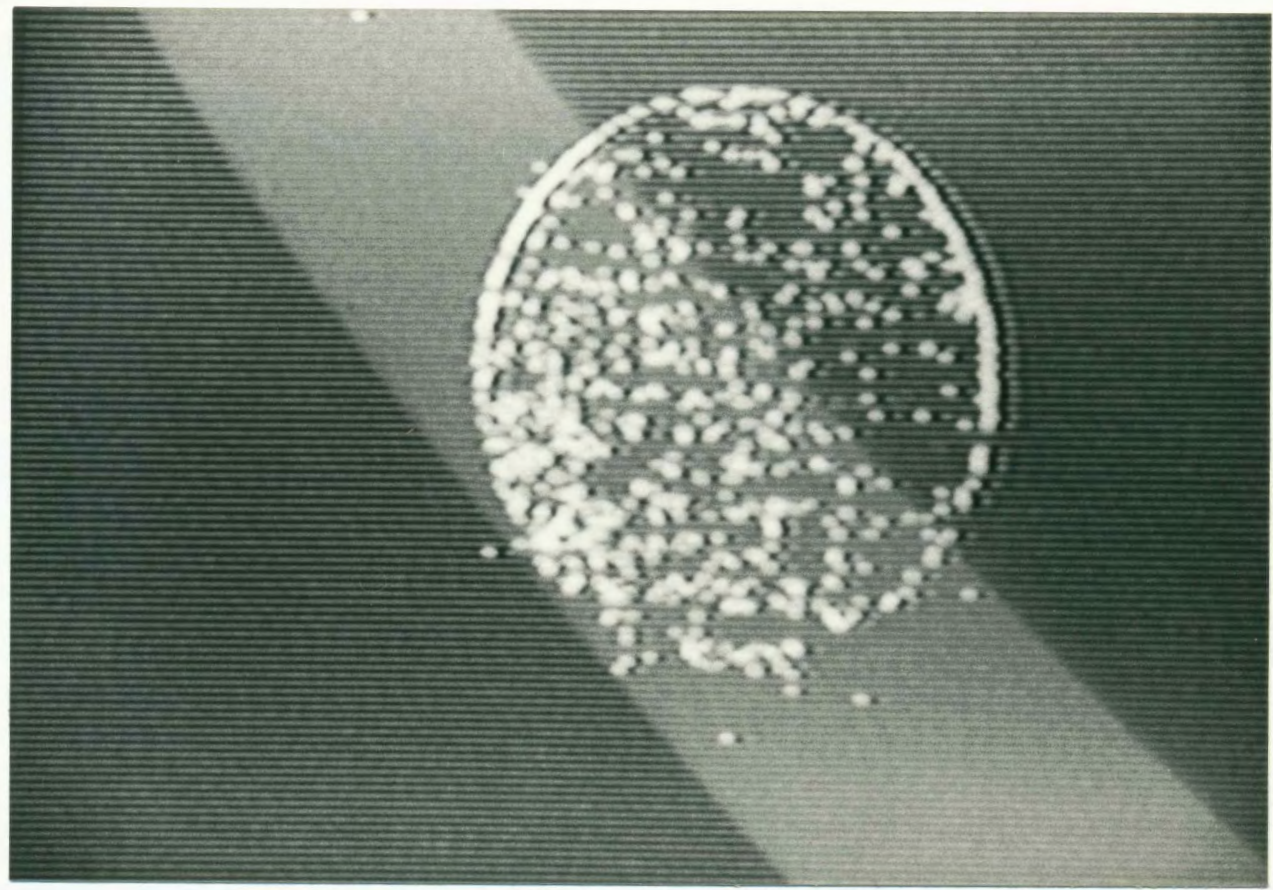

Figure 32. Contour image of edge detected by Roberts edge filter 


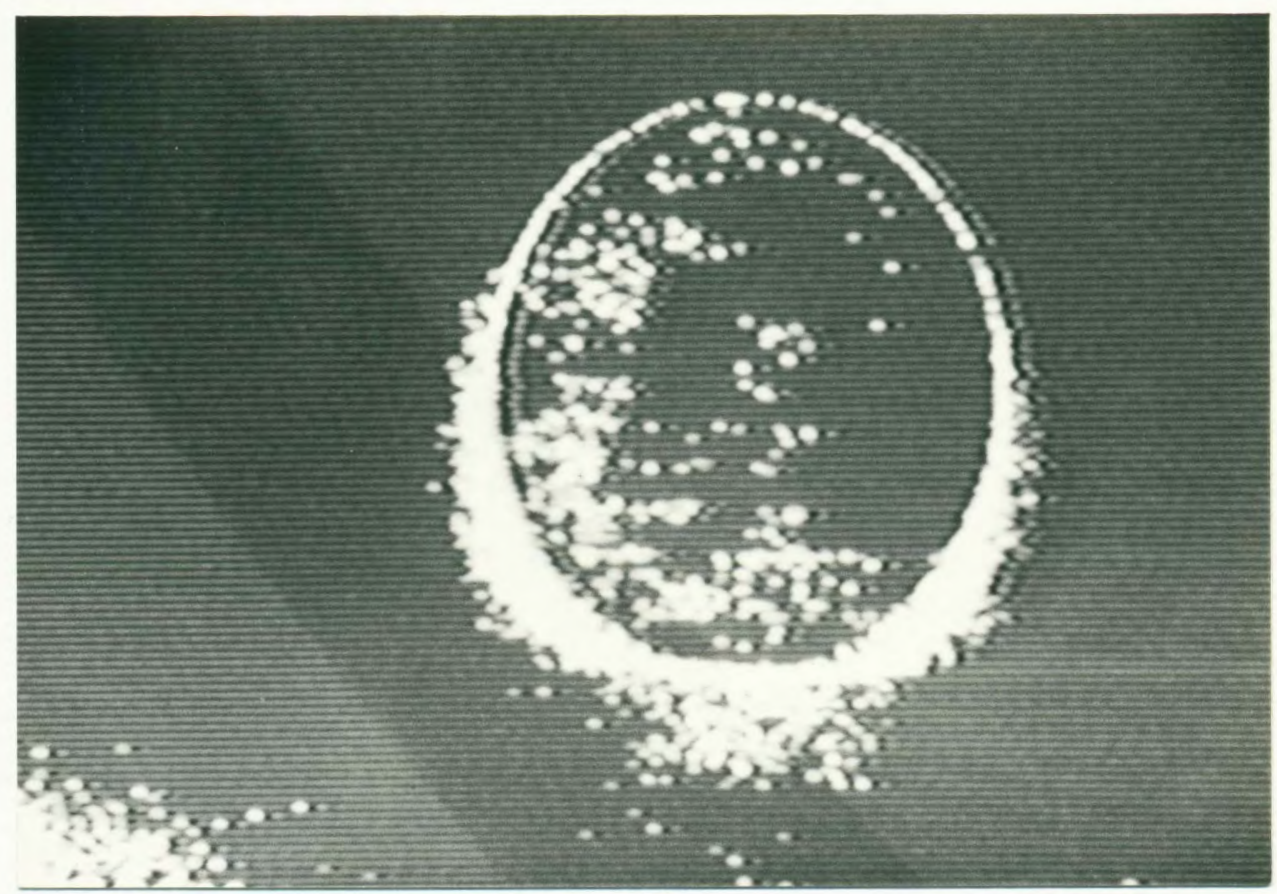

Figure 33. Contour image extracted by the threshold(b) method

\section{$\underline{3.6 .2 \text { Rectangular Objects }}$}

The original gray scale image of the wood block is shown in Figure 34. The Signal to Noise ratio is $S N R=156$. Different than in the case of circular edges, the geometry of a straight line is related to the geometry of a CCD element. A straight line edge occurs perfectly on a binary contour image if the edge line is exactly parallel to the rows and columns of the CCD elements. Otherwise, a straight line contour with steps is detected and no perfect contour image presentation can be obtained. The threshold(a) method (Figure 35) detects a closed contour. The upper horizontal edge is rough. Since pixels of the textured surface have the same intensity as the background, no threshold can be found which completely divides the set of object pixels from the set of background pixels. The upper horizontal edge is detected as a jagged 
line. By preprocessing the gray scale image with the local statistic filter these problems disappear. A sharp closed straight line contour is detected (Figure 36). Figures 37, 38, and 39 show the result of the variance $(n)$ method using $3 \times 3,5 \times 5,7 \times 7$, neighboring pixels. With increasing $n$ the occurrence of noise pixels is reduced. For the same reason as in the case of detecting a circle, Roberts edge filter (Figure 40) and the threshold(b) methods (Figure 41) fail.

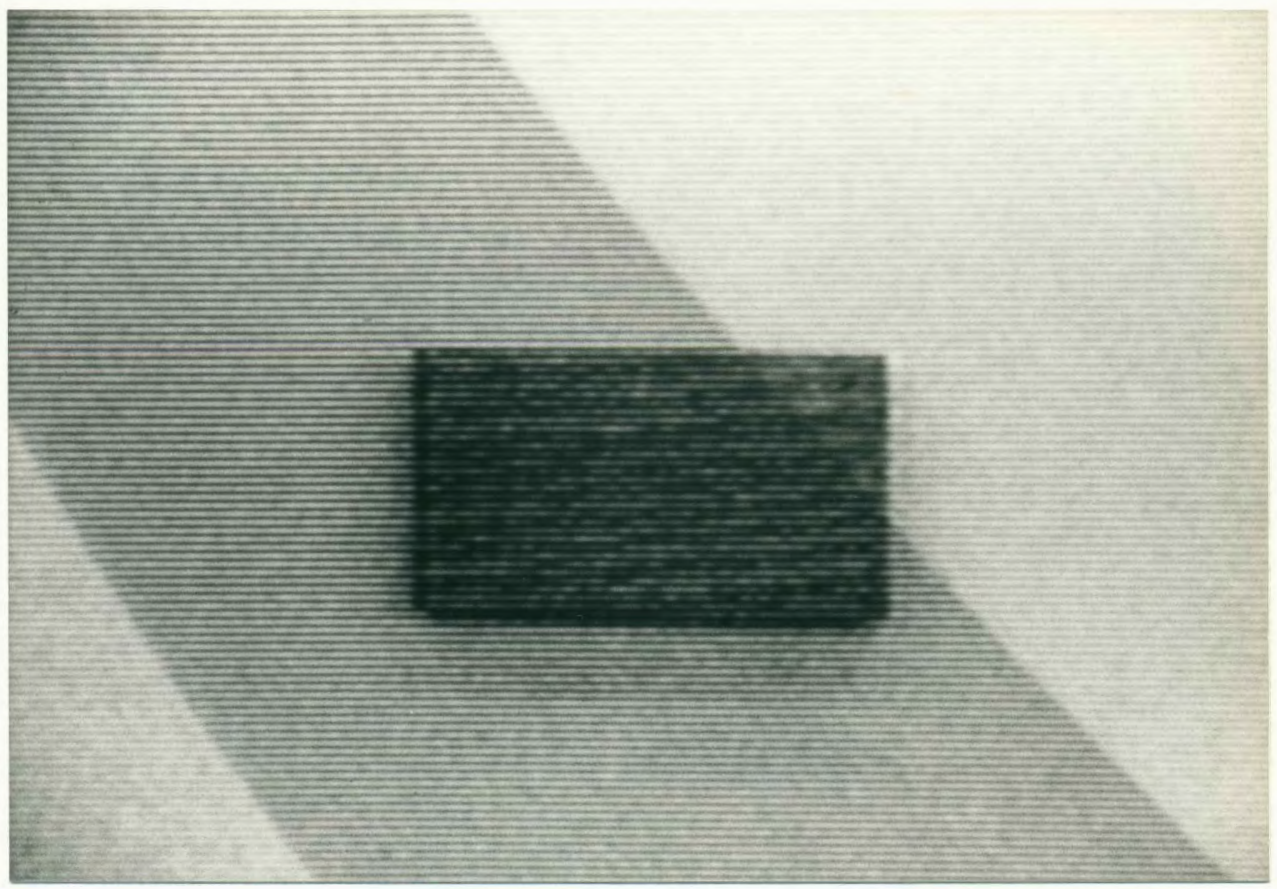

Figure 34. Gray scale image of rectangular shaped block of wood 


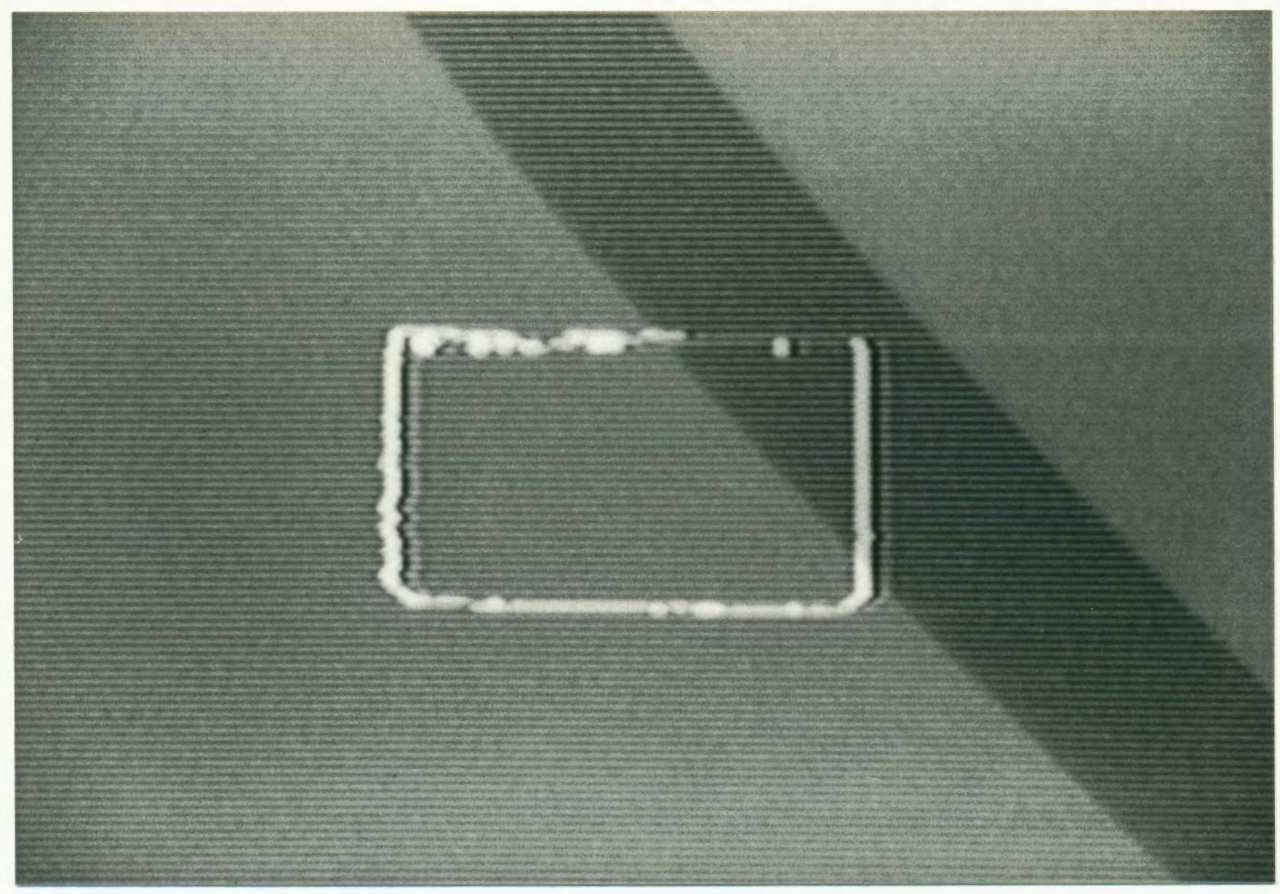

Figure 35 . Contour image extracted by the threshold(a) method

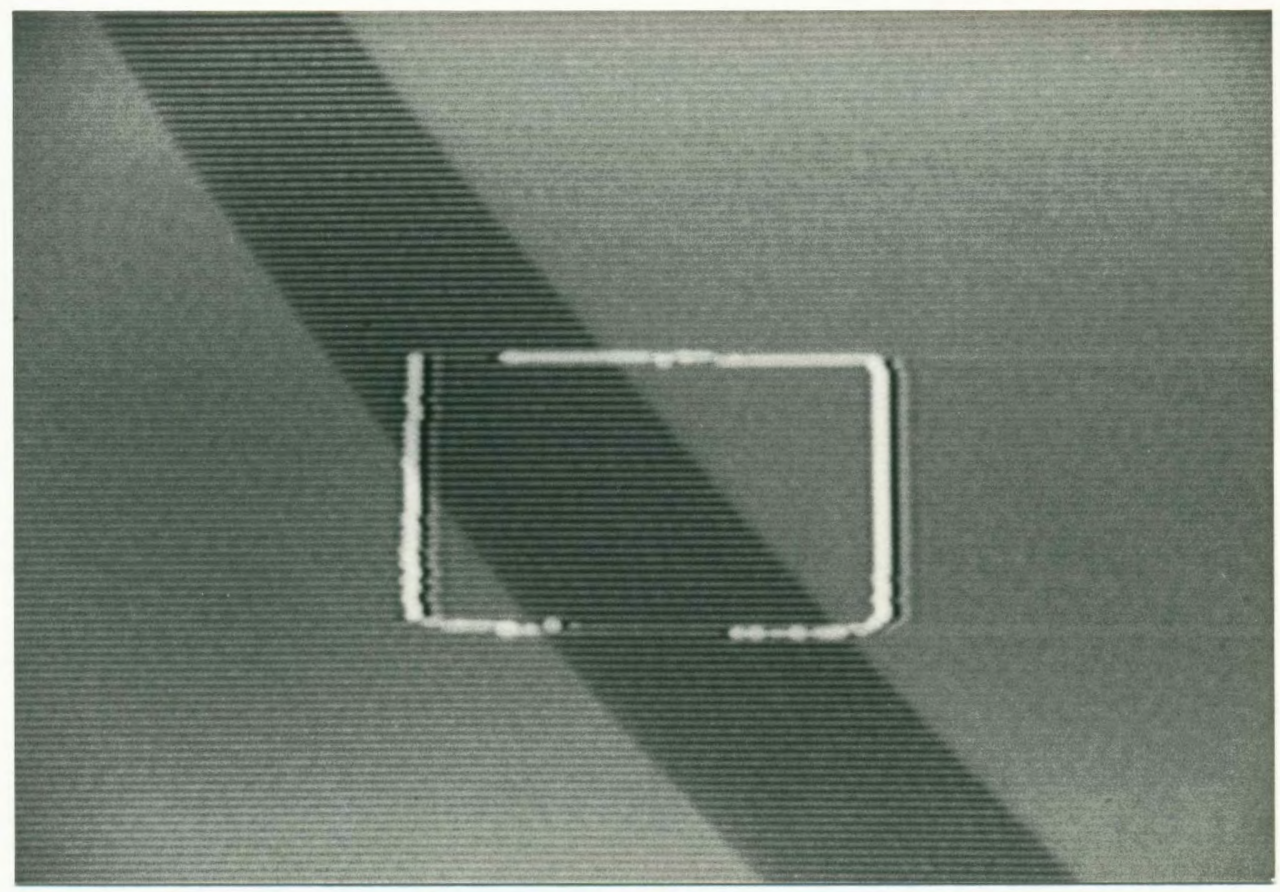

Figure 36 . Contour image extracted by the local statistic method 


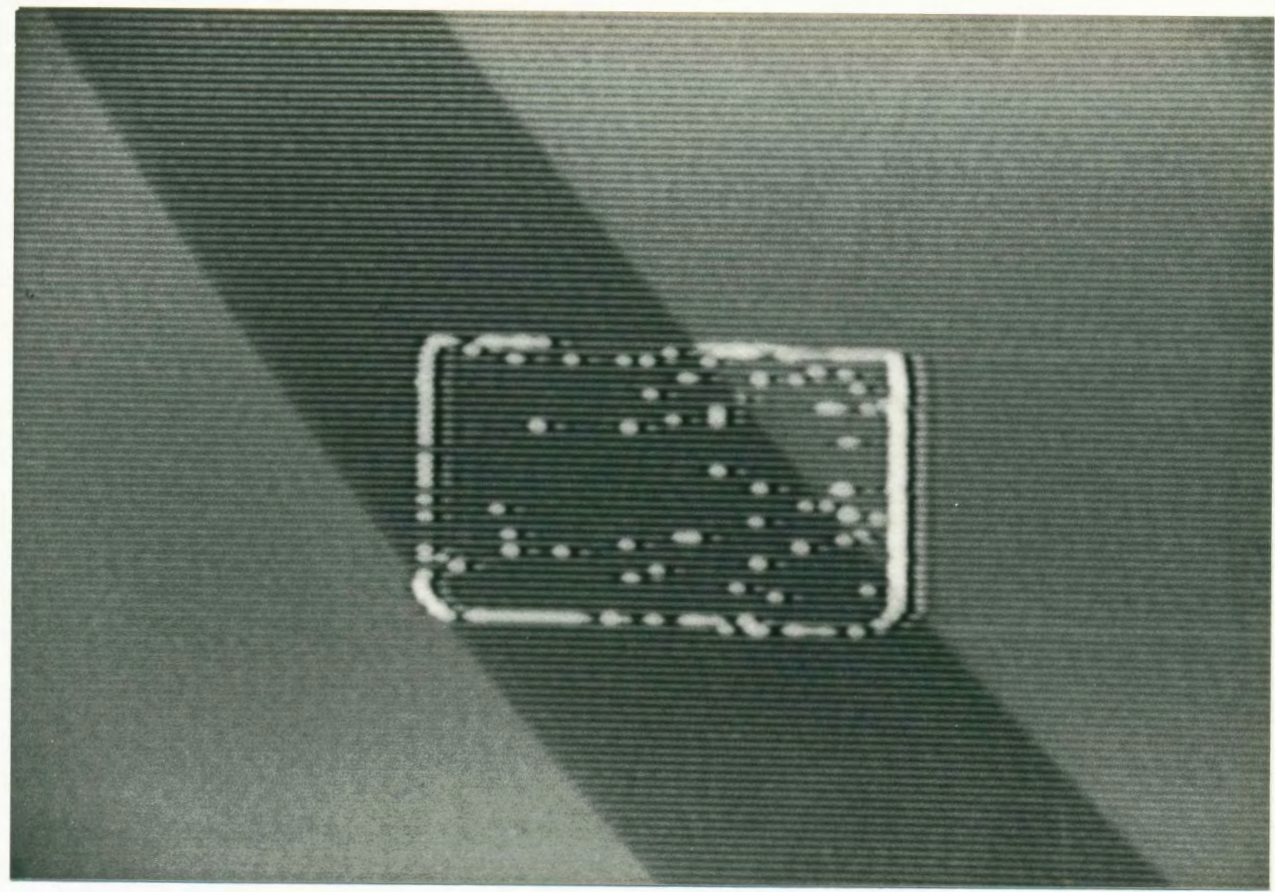

Figure 37 . Contour image extracted by the variance(3) method

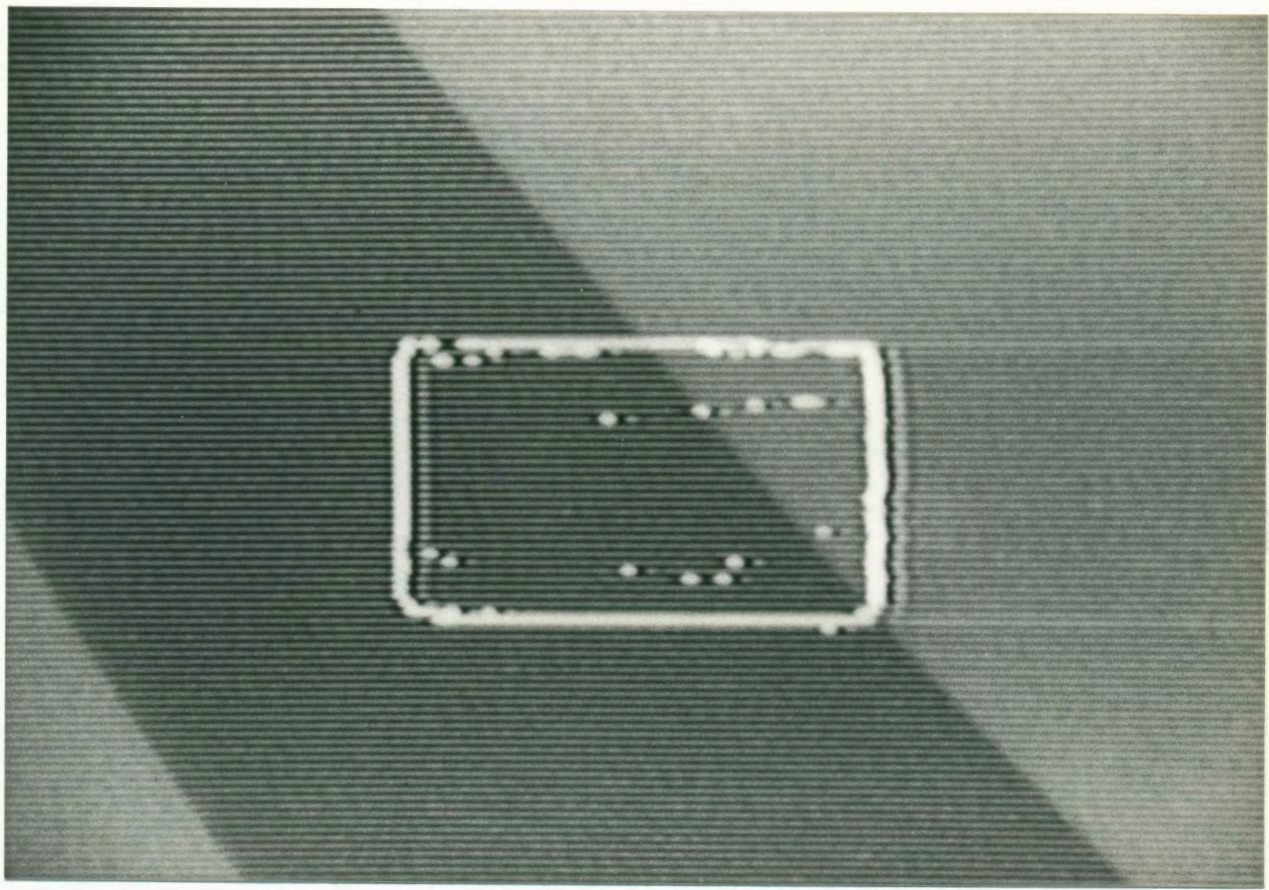

Figure 38. Contour image extracted by the variance(5) method 


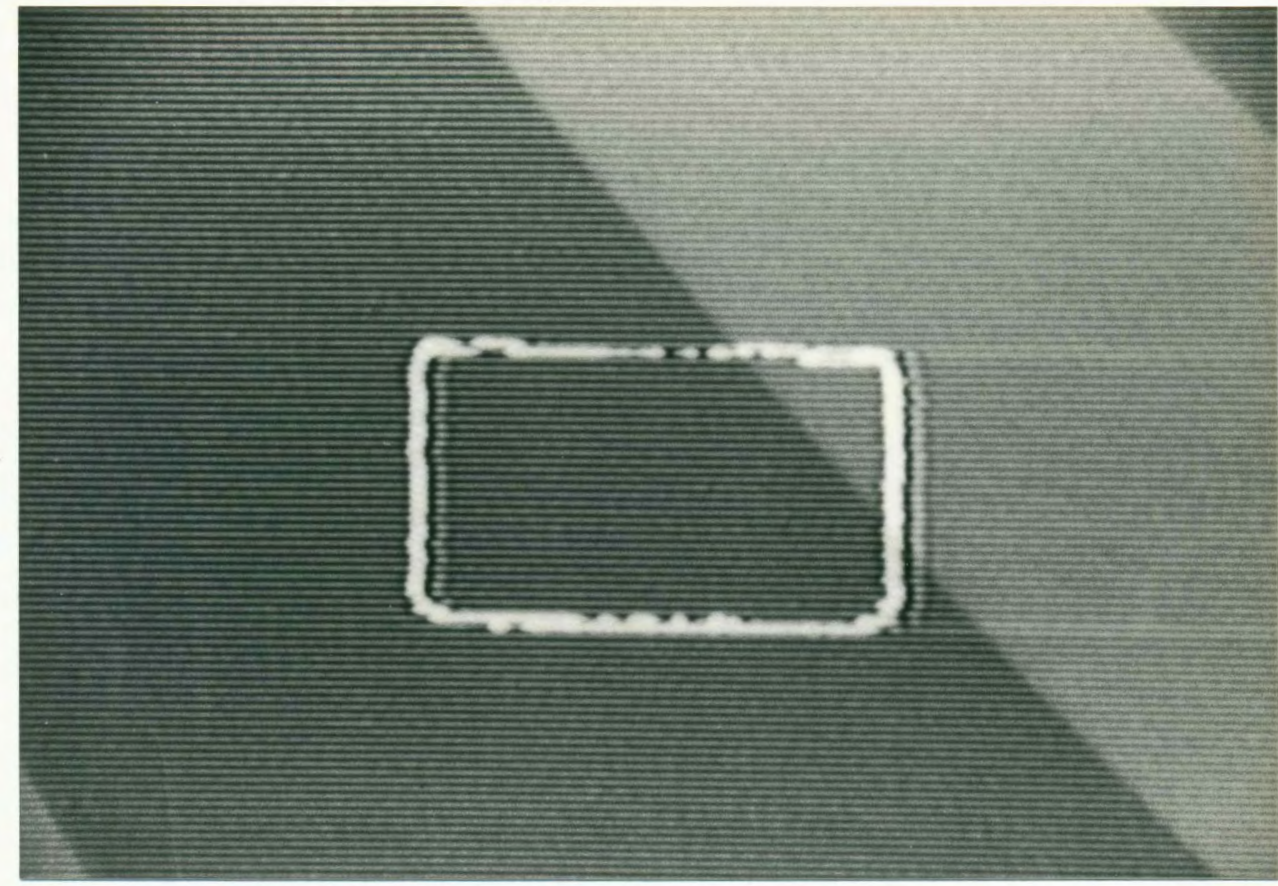

Figure 39. Contour image extracted by the variance(7) method

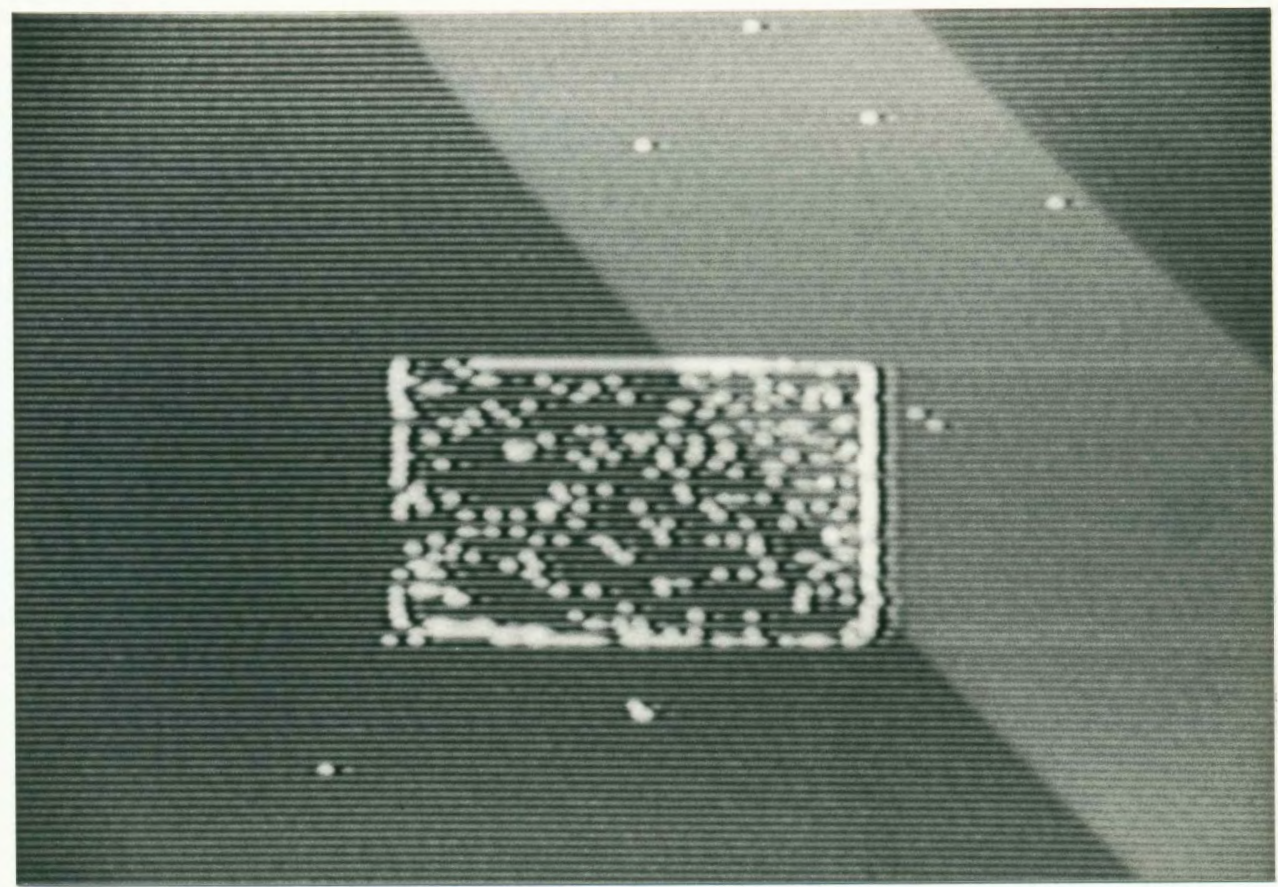

Figure 40 . Contour image of edge detected by Roberts edge filter 


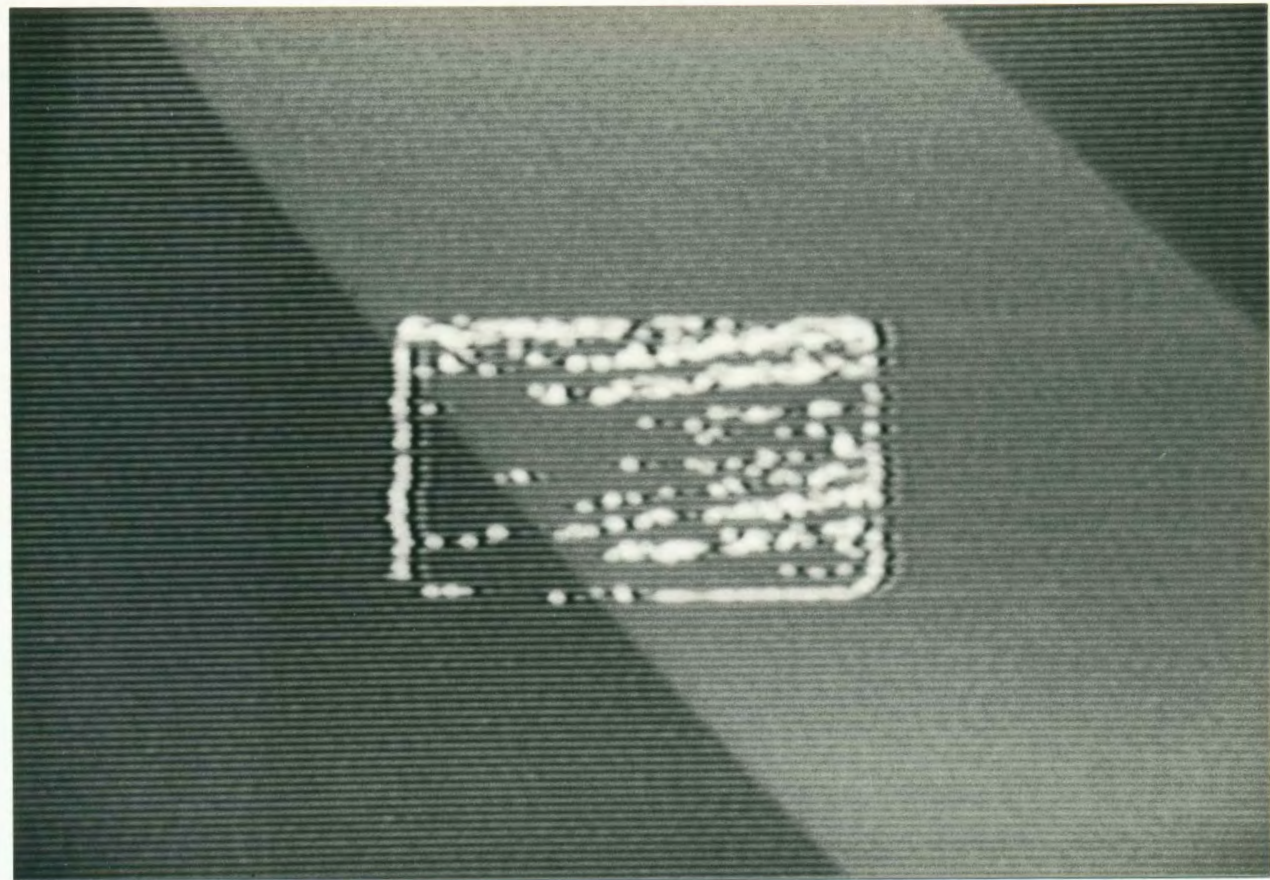

Figure 41. Contour image extracted by the threshold(b) method

\subsection{STATEMENT OF COMPARISON}

The tested algorithms can basically be divided into 3 groups.

- threshold(a), local statistic filter

- variance(n), Roberts edge filter

- threshold(b)

Threshold(a) and the local statistic method both result in a binary image, from which the edge contour is extracted. For increasing noise level no exact threshold can be found. By preprocessing the image with the local statistic filter, noise pixels are smoothed by averaging, using a subset of neighborhood pixels, which represent the most likely side of the edge where the pixel to be filterd belongs to. Therefore the local statistic filter performs far better for images with low SNR 
and textured edges than thresholding(a).

The variance(n) method uses, like the local statistic method, larger areas of neighborhood pixels to make a decision about an edge but without preprocessing the image. The larger the window size, $n$, the more capable is the method for detecting edges in images with low SNR. Another advantage of increasing $\mathrm{n}$ is the smoother representation of the edge. Since noise pixels are rejected, the variance threshold can be optimized with regard to a sharp representation of the edge and a closed contour. Edges of textured objects can be detected for large $n$, as well, but still the local statistic method is preferable for this task, due to the use of gradient direction information. Processing the next larger size of neighborhood increases the computation time by a factor of $(n / n-1)^{2}$. The variance window size $n$ should not be too large, otherwise sharp edges cannot be detected detailed enough. The change of the gradient of the edge line within the window should be small. Roberts filter is similar to the variance( $n)$ method, but uses less pixels to decide whether or not a point belongs to an edge or not. Based on 2 cross gradients of 4 pixels the new pixel value is calculated. These are less pixels than the variance(3) methods uses to establish a measure of edge strength. Therefore Roberts Edge filter is more sensitive to noise and texture. Roberts edge filter should be used for images with high signal to noise ratio. Sharp edges are detected more accuratly with this method than with filters using large window sizes.

Threshold(b) is a weak method for the purpose of dimensional inspection if the image is noisy or shows textured parts. This method 
can only be used for accurate measurements for images with high SNR. However the computation time for the detection process is minimum because the operation is performed by hardware and no contour has to be processed as in the case of threshold(a). This method should be used for inspection cycles with high frequency. As threshold(a), threshold(b) relates only to the intensity value of one pixel without regard to any spatial relationships. Threshold(a) is a stronger method in detecting edges, because the contour following algorithm is only influenced by noise pixels occurring next to the edge. Threshold(b), Roberts edge filter, and the variance method detect noise pixels occurring in the area of measurement besides the extracted edge pixels. This results in weak geometric stability as soon as noise pixels are enhanced.

The methods were not compared with regard to the computational effort for the process of edge detection. The processing time changes with the type of image. First, criteria for standard images have to be developed to allow a successful benchmarking operation. To give the reader a general idea, a ranking of the methods is given in increasing order for the computation time: threshold(b), threshold(a), Roberts edge filter, variance(3), variance(5), local statistic filter. 
CHAPTER IV

\section{CAIIBPATION}

Besides choosing the right inage analysis method for a certain type of application, the user of a vision system has to calibrate the camera setup. If the plane of the camera iens is not parallel to the circle surface, a perfect circle would appear as an ellipse on the image. This effect has to be taken into account and compensated for. Therefore, calibration is another important factor in determining the accuracy of a vision system.

Calibration is defined in The Manual of Photogrammetry [22] as:

The act or process of determining certain specific measurements in a camera or other instrument or device by comparison with a standard, for use in correcting or compensating errors for purposes of record.

To obtain knowledge about the real dimensions of a part which has been measured, the image information about this part has to be changed into a real world representation. This is done by transforming pixel information from the image coordinate system to the real world coordinate system. To be able to do this requires knowledge about the transformation parameters. The process of finding these parameters is called calibration.

To calibrate a vision system, the following equipment information must be acquired: 
1) highly accurate calibration pattern coordinates (real world knowledge)

2) representation of these coordinates in the image plane

3) a model to calculate the calibration parameters.

\subsection{A MODEL}

The calibration process is done in four steps (Figure 42). The world coordinates of a highly accurate pattern are transformed into the camera coordinate system (step 1), and then into the coordinate system of the image plane (step 2). Compensating for distortion transforms the points into the form where the CCD elements of the camera produce the signal (step 3). Then, the coordinates are transformed into computer coordinates (step 4), which are displayed on the monitor of the vision system.

The coordinate system setup is shown in Figure 43. All coordinate systems are right-handed. The $z$-axis of the world coordinate system points in the opposite direction of the z-axis of the camera coordinate system. The $x, y$ planes of the camera and the image coordinate systems are parallel. Their origins have the same $x, y$ coordinates and the distance between the planes is $f$, the focal length.

\subsubsection{Trarsformation from world to camera coordinate system}

To transform coordinates from the world to the camera coordinate system, the points have to be translated by 


\section{points in world coordinate system}

points in camera coordinate system

points in image plane coord.system-undistorted

\section{points in image plane coord.system-distorted}

points in screen coord.system-pixel units

Figure 42. Calibration in 4 steps. 


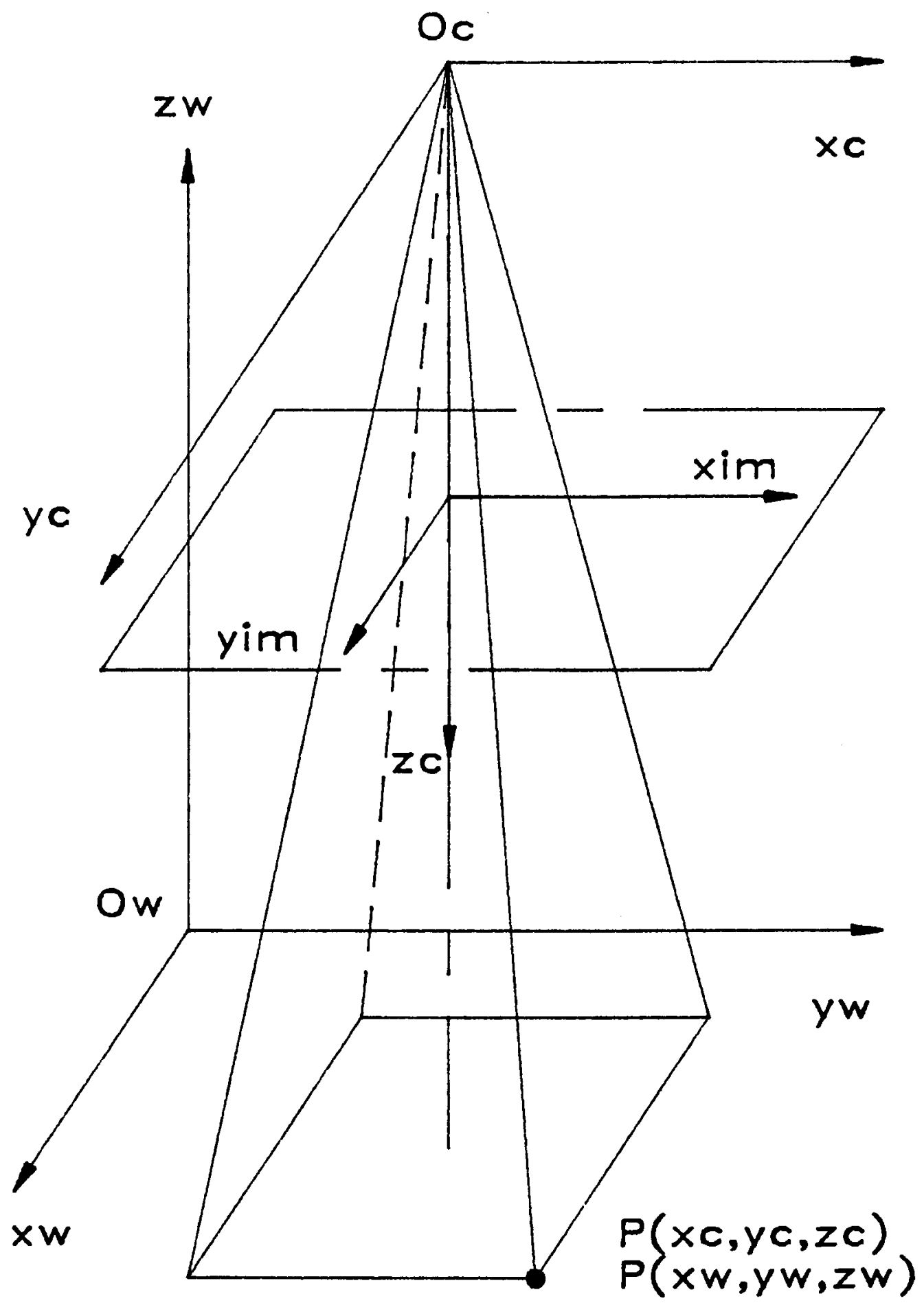

Figure 43. Orientation of coordinate system for calibration. 


$$
T=\left[\begin{array}{c}
T \\
x \\
T \\
T \\
z
\end{array}\right]
$$

where $T_{x}, T_{y}$, and $T_{z}$ are the coordinates of the origin of the world coordinate system in the camera coordinate system representing the translation vectors in $x, y, z$ direction.

The translated points are rotated by using the rotation matrix

$$
R=\left[\begin{array}{lll}
r_{1} & r_{2} & r_{3} \\
r_{4} & r_{5} & r_{6} \\
r_{7} & r_{8} & r_{9}
\end{array}\right]
$$

where $r l-r_{g}$ are the components of the rotation matrix, as a function of the rotation angle $\theta, \phi, \psi$ of the $x, y, z$ axis.

This results in the composite transformation matrix,

$$
T=\left[\begin{array}{cccc}
r_{1} & r_{2} & r_{3} & T \\
r_{4} & r_{5} & r_{6} & T \\
r_{7} & r_{8} & r_{9} & T \\
0 & 0 & 0 & 1
\end{array}\right]
$$

The transformation equation results in

$$
P_{c}=T_{w c} \cdot P_{w} \text {, where }
$$

$P_{c}=$ point in camera coordinate system, and

$P_{w}=$ point in world coordinate system.

The transformation matrix in the form of rotation angles yields

$$
P_{c}=R_{z}(\psi) \cdot R_{y}(\phi) \cdot R_{x}(\theta) \cdot T_{R} \cdot P_{w} \text {, where }
$$


$\theta, \phi, \psi$ are the Euler angles, known as yaw, pitch, and roll, and

$$
\begin{aligned}
& R_{x}(\theta)=\left[\begin{array}{cccc}
1 & 0 & 0 & 0 \\
0 & \cos \theta & -\sin \theta & 0 \\
0 & \sin \theta & \cos \theta & 0 \\
0 & 0 & 0 & 1
\end{array}\right] \\
& R_{y}(\phi)=\left[\begin{array}{cccc}
\cos \phi & 0 & \sin \phi & 0 \\
0 & 1 & 0 & 0 \\
-\sin \phi & 0 & \cos \phi & 0 \\
0 & 0 & 0 & 1
\end{array}\right]
\end{aligned}
$$

and

$$
R_{z}(\psi)-\left[\begin{array}{cccc}
\cos \psi & -\sin \psi & 0 & 0 \\
\sin \psi & \cos \psi & 0 & 0 \\
0 & 0 & 1 & 0 \\
0 & 0 & 0 & 1
\end{array}\right]
$$

The composite rotation matrix $R=R_{z}(\psi) \cdot R_{y}(\phi) \cdot R_{x}(\theta)$ results in:

$$
R=\left[\begin{array}{cccc}
\cos \psi \cos \phi & -\sin \psi \cos \theta+\cos \psi \sin \phi \sin \theta & \sin \psi \sin \theta+\cos \psi \sin \phi \cos \theta & 0 \\
\sin \psi \cos \phi & \cos \psi \cos \theta+\sin \psi \sin \phi \sin \theta & -\cos \psi \sin \theta+\sin \psi \sin \phi \cos \theta & 0 \\
-\sin \phi & \cos \phi \sin \theta & \cos \phi \cos \theta & 0 \\
0 & 0 & 0 & 1
\end{array}\right]
$$

For the coordinate system setup in this project, the Euler angles are approximately:

$$
\begin{aligned}
& \mathrm{x}: \theta \approx 180^{\circ} \\
& \mathrm{y}: \phi \approx 0^{\circ} \\
& \mathrm{z}: \psi \approx 90^{\circ}
\end{aligned}
$$

and yield the rotation matrix 


$$
R \approx\left[\begin{array}{ccc}
0 & 0 & 0 \\
1 & 0 & 0 \\
0 & 0 & -1
\end{array}\right]
$$

\subsubsection{Transformation from camera to image plane coordinate system for}

\section{undistorting optical systems.}

Using the model of the pinhole camera geometry, in other words, the fact that the $\mathrm{x}, \mathrm{y}$ plane of the camera coordinate system is parallel to the image plane but translated by $|f|$, the focal length, the following relationship can be established:

$$
\frac{x_{i m}}{x_{c}}-\frac{|f|}{|T|}
$$

where $T_{z}$ is the translation component of the camera coordinate system in $z$-direction with respect to the origin of the world coordinate system. $\mathrm{X}_{1 \mathrm{~m}}, \mathrm{Y}_{\mathrm{im}}$ are the coordinates of a point in the image coordinate system, obtained by intersecting the $\mathrm{x}, \mathrm{y}$ plane of the image coordinate system with a ray, starting from a point, with the coordinates $x_{c}, y_{c}$ expressed in the camera coordinate system.

This results in:

$$
x_{\text {im }}=x_{c} \frac{|f|}{|T|}
$$

and similarly in:

$$
Y_{1 \mathrm{II}}=Y_{c} \frac{|\mathrm{f}|}{\mid \begin{array}{c}
\mid \mathrm{T} \\
z
\end{array}}
$$


4.1.3 Transformation of points from undistorted to distorted image coordinates.

Two kinds of lens distortion can be observed, radial and tangential. The radial lens distortion can be further divided in barrel distortion and pin cushion distortion. For the fundamental theory of distortion and the derivation of equations, the Manual of Photogrammetry[22] is recommended. The radial lens distortion is modeled as an odd-powered polynomial [22],

$$
\Delta \mathrm{r}-\mathrm{k}_{1} \cdot \mathrm{r}^{3}+\mathrm{k}_{2} \cdot \mathrm{r}^{5}+\mathrm{k}_{3} \cdot \mathrm{r}^{7}+\ldots
$$

using the relationship

$$
\begin{aligned}
& \mathrm{x}^{\prime}=\mathrm{x}-\mathrm{x} \frac{\Delta \mathrm{r}}{\mathrm{r}} \\
& \mathrm{y}^{\prime}=\mathrm{y}-\mathrm{y} \frac{\Delta \mathrm{r}}{\mathrm{r}}
\end{aligned}
$$

where $x^{\prime}, y^{\prime}$ are the corrected image coordinates of the undistorted image. Tsai [23] proposes that only $K$, the radial lens distortion coefficient, should be considered and that tangential distortion can be neglected.

\subsubsection{Final Transformation into Computer Coordinate System.}

To obtain the computer coordinates, the calibration points have to be transformed from distance units to pixel units. Figure 44 shows the process of scaling and transformation into computer coordinates. The image of the CCD element is digitized and scaled to fit the frame buffer. Since the origin of the image plane is placed in the center of the computer coordinate system, $C_{x}, C_{y}$, the scaled points in computer 
coordinate system are translated by $\mathrm{C}_{x}, \mathrm{C}_{\mathbf{y}}$.

The scale factor to transform distance to pixel units is

$\frac{\text { distance in } x \text { of computer coordinates }}{\text { distance in } x \text { in undistorted image coordinate system }}=\frac{1}{\mathrm{~d}_{\mathrm{x}}^{*}}$

setting the scale factor in a similar way for $y$, yields the transformation equations

$$
\begin{aligned}
& x_{f}=\frac{1}{d^{*}} \cdot x^{\prime}+c_{x} \\
& y_{f}=\frac{1}{d_{y}^{*}} \cdot y^{\prime}+c_{y}
\end{aligned}
$$

where $x_{f}, y_{f}$ are the computer image coordinates.

Since the number of pixels of the CCD element $\mathrm{N}_{\mathrm{cx}}$ are mapped onto the frame buffer with the computer sampling $N_{f x}$,

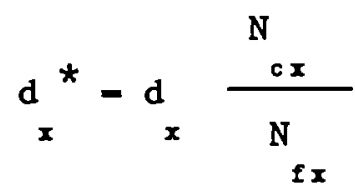

where $d_{x}$ is the distance of two adjacent CCD elements in $x$ direction. Whereas in $y$ direction, every row is processed by vertical scanning and therefore,

$$
d_{y}{ }^{*}=d_{y}
$$

and $d_{y}$ is the distance of two adjacent CCD elements in $y$ direction. 

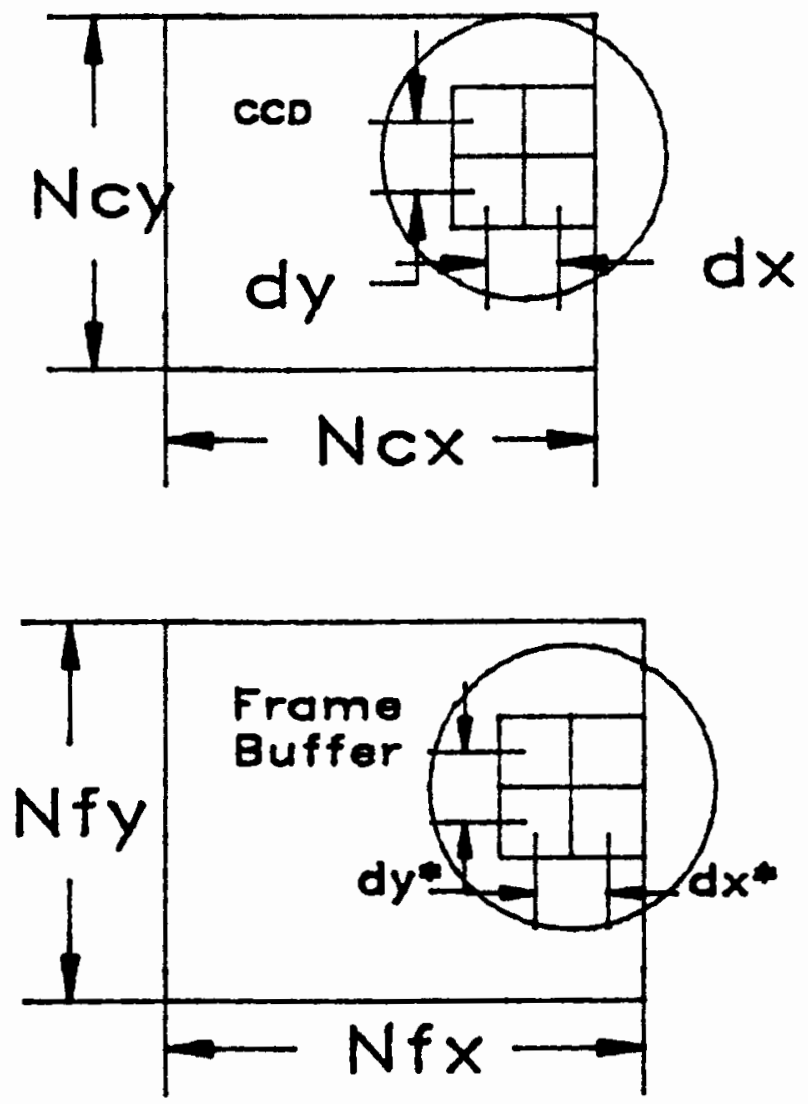

$$
\begin{gathered}
d x^{*}=d x(\operatorname{Ncx} / N f x) \\
d y^{*}=d y
\end{gathered}
$$

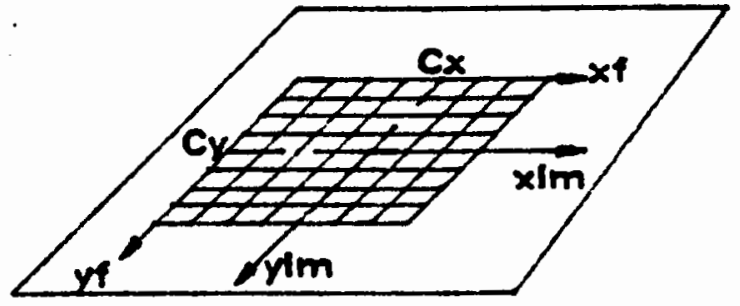

$$
\begin{aligned}
& x f=x^{\prime} / d x^{*}+c x \\
& y f=y^{\prime} / d y^{*}+c y
\end{aligned}
$$

Figure 44.Scaling and transformation into computer coordinate system. 
For the CCD camera used in this project, $N_{c x}=512, N_{c y}=492, d_{x}=17 \mu$ and $d_{y}-13 \mu$. The signal of the 512 elements in $x$ direction of the CCD element is digitized on 640 pixels. From the 492 element lines in $y$ direction of the CCD element, only $\mathrm{N}_{f y}-480$ are used, and the signal produced by the rest is not used to form the image.

\subsection{COMPUTING THE CALIBRATION PARAMETERS}

\subsubsection{Nonlinear Optimization}

In 4.1 (A Mode1), formulas relating the world coordinate to the computer coordinate system are derived. The eight unknown parameters are found by numerical calculations. The numerical solution to the calibration problem can be found by the use of nonlinear optimization of the calibration parameters $\theta, \phi, \psi, T_{x}, T_{y}, T_{z}, k_{1}, f$. The calibration points in the world coordinate system are transformed in four steps into the computer coordinate system using the determined equations. At this point, a good initial guess is required. The squared sum of the difference between the observed pixels and the calculated values represented in the computer coordinate system is minimized by optimizing the calibration parameters.

The disadvantage of using nonlinear optimization is that the computation time to optimize the variables is too long for practical purposes. In addition, by using an initial guess, the minimization function will most likely diverge.

\subsubsection{Two-stage solution by Tsai [23]}

Tsai developed a useful method to compute the calibration parameters. This method was studied and implemented during this 
project. Tsai's model differs from the model derived above, in that in equation $(*)$, an uncertainty factor $S_{x}$ is introduced to compensate for timing mismatch between image acquisition and camera scanning hardware. For the equipment used, $s_{x}=1.042$. A short outline of how this two stage calibration is implemented is described below.

The calibration takes into account the parameters of the rotation matrix $r_{1}$ to $r_{g}$, the component of the translation matrix $T_{x}, T_{y}, T_{z}$, the focal length $f$ and the radial distortion coefficient $k_{1}$.

By introducing the "Radial Alignment Constraint," a solution for

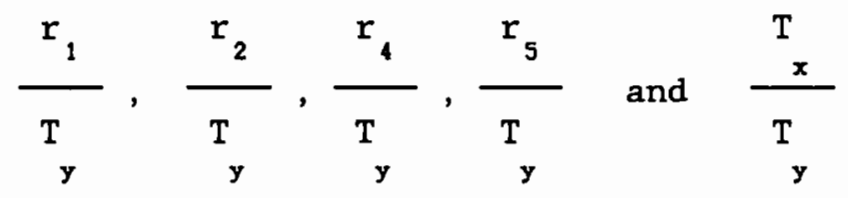

can be obtained by solving an overdetermined system of linear equations.

\section{Radial alignment constraint}

Because of the fact that the $x, y-p l a n e$ of the camera coordinate system and the $x, y-p l a n e$ of the image coordinate system of the model are parallel, the product of the two vectors, representing a point in the world and the image coordinate system, is $\left(x_{i m}, y_{i m}\right) x\left(x_{c}, y_{c}\right)=0$. This results in an overdetermined set of linear equations. To solve this overdetermined system, the method of singular value decomposition [24] has been used in this project. This method gives very accurate results and has the advantage of obtaining the closest solution in the case of an 111-conditioned matrix.

Using the fact that an orthonormal $3 \times 3$ matrix has a unique $2 \times 2$ submatrix, differing by a scale factor no other than \pm 1 , the absolute value of $\mathrm{T}_{\mathrm{y}}$ is calculated. The rest of the components of the rotation 
matrix $r_{3}, r_{6}, r_{7}, r_{8}, r_{9}$ are solved using the orthonormal property. Since the focal length is $f>0$ and $T_{z}>0$, the coordinates in world and image plane must have the same sign. If this is not the case, the sign of $T_{y}$ must be changed. A second overdetermined system of equations is solved for $f$ and $T_{z}$. The calibration plane and image plane must not be exactly parallel since the set of equations would then become linearly dependent. If the focal length is $f<0$, then the signs of $r_{3}, r_{6}, r_{7}, r_{8}$ have to be reversed and $f$ and $T_{z}$ are calculated again. By using optimization, $f, T_{2}$, and $k_{1}$ are determined. For this purpose, the conjugate gradient method of Polak-Ribiere has been implemented [24].

The source code of the implemented calibration algorithm is included in Appendix $F$. Because a highly accurate calibration pattern was not available at the time of testing time, the algorithm was tested on pseudo pattern with 12 calibration points. The algorithm was verified with regard to this accuracy. The computation time for the numerical solution of the calibration on a PC-AT $12.5 \mathrm{MHZ}$ was approximately 2 seconds. 
CHAPTER V

\section{CONCLUSIONS AND RECOMMENDATIONS}

\subsection{CONCLUSIONS}

Different image analysis methods have been studied and compared. But how do the results of the comparison of the methods and the calibration related to the overall performance of a vision system for dimensional inspection? Figure 45 shows three main areas, which determine the accuracy of the measurement. These three areas are:

- Image analysis methods used for measurements

- Calibration and its accuracy

- Resolution of the system

\section{Image Analysis Methods}

The main emphasis in this research project was on the comparison of different image analysis methods. A decision model to select the right method for a certain application has been proposed. The experimental results show the range where the tested methods perform we11, and the range where they fail. The quantitative real world experiments illustrate the conclusions drawn out of the simulation experiments. With the result of the simulation in Chapter III, it is possible to choose the right method for an application or specific image scene. For accurate dimensional inspection, the contrast, i.e. the difference between the part and the background intensity should be as large as possible. High contrast inspection scenes can be achieved by 


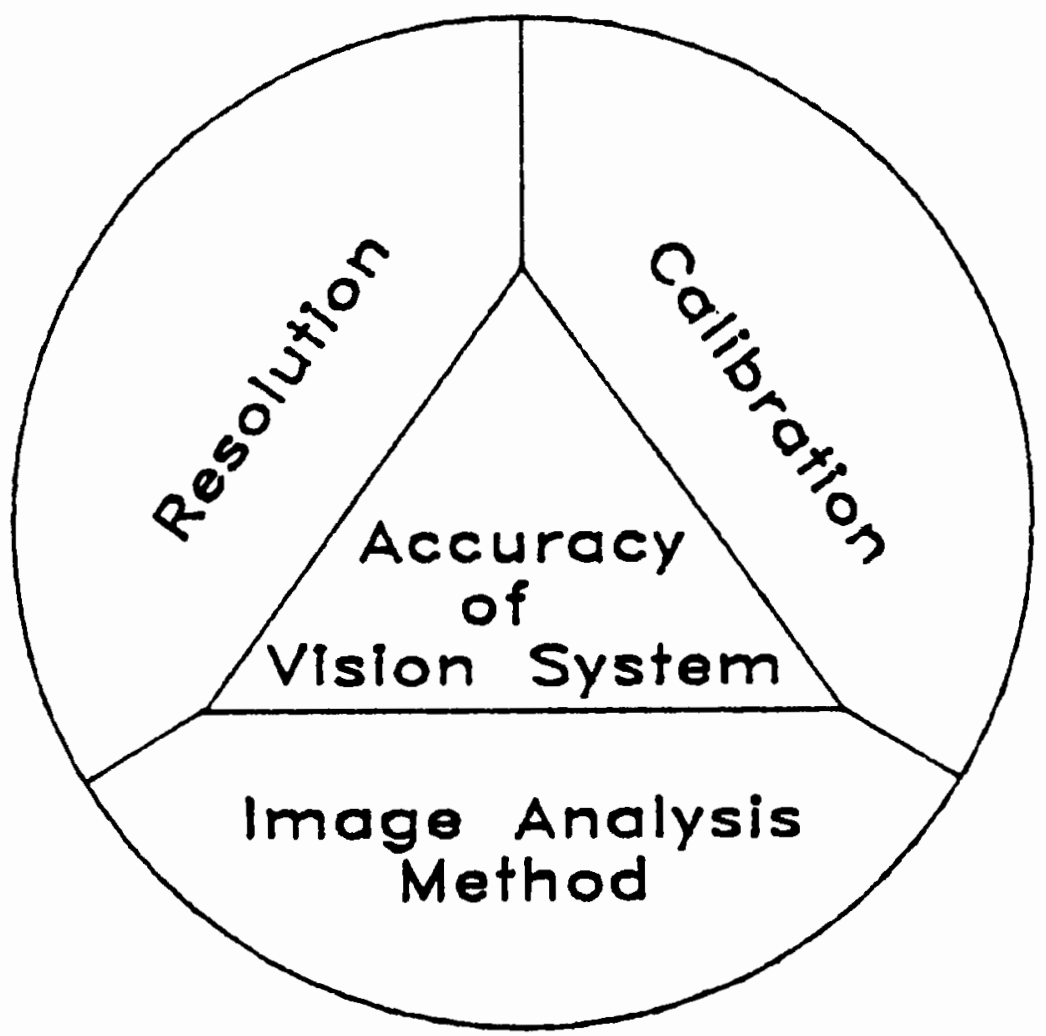

Figure 45.Accuracy of vision systems for dimensional inspection

using a diffuse light source mounted below the part. In applications where this type of lighting cannot be established, diffuse overhead light should illuminate the scene as uniformly as possible. In general it can be said, that the lower the Signal to Noise ratio of a scene, the more difficult is it to detect the features of the image, and the higher the computational effort. Every image analysis method introduces errors in terms of the qualitative measures such as pixel stability, geometric stability and repeatability of the outcome of the measurement.

\section{Calibration}

Image coordinates, in units of pixels have to be transformed to world coordinates in units of dimensional measures, to obtain "Real 
World Knowledge" of the detected edge of the part. For this purpose a calibration algorithm has been implemented. Its computational speed and the general setup of the calibration model suggest its use in the field of robotic vision system, where the calibration parameters have to be calculated with every movement of the robot. As the image analysis method, the process of calibration contributes errors to the overall accuracy of the system. With increasing number of calibration points the error decreases [23].

\section{Resolution of the system}

The resolution of the system is expressed in terms of dimension per pixel and is the major factor in determining the accuracy of the dimensional inspection process with vision systems. Whether the measurement of a circle incorporates sub-pixel accuracy should be determined by testing the final accuracy of the measurement by taking into account the three main influence factors (Figure 45). In addition, the errors of the transformation of a circle from continuous to discrete space must be investigated.

One final experiment shows a trend towards the use of subpixel accuracy measures. A binary image of circles with different sizes were generated and the diameter was detected by thresholding(a). Figure 46 shows the ratio of the generated difference to detected difference versus the generated difference for different circle sizes in units of pixels. The approximate curve of the experimental points shows a sufficient response of the measurement system down to 0.5 pixel differences of the diameter. Gray scale systems should be even more sensitive due to the use of multiple intensity levels. 


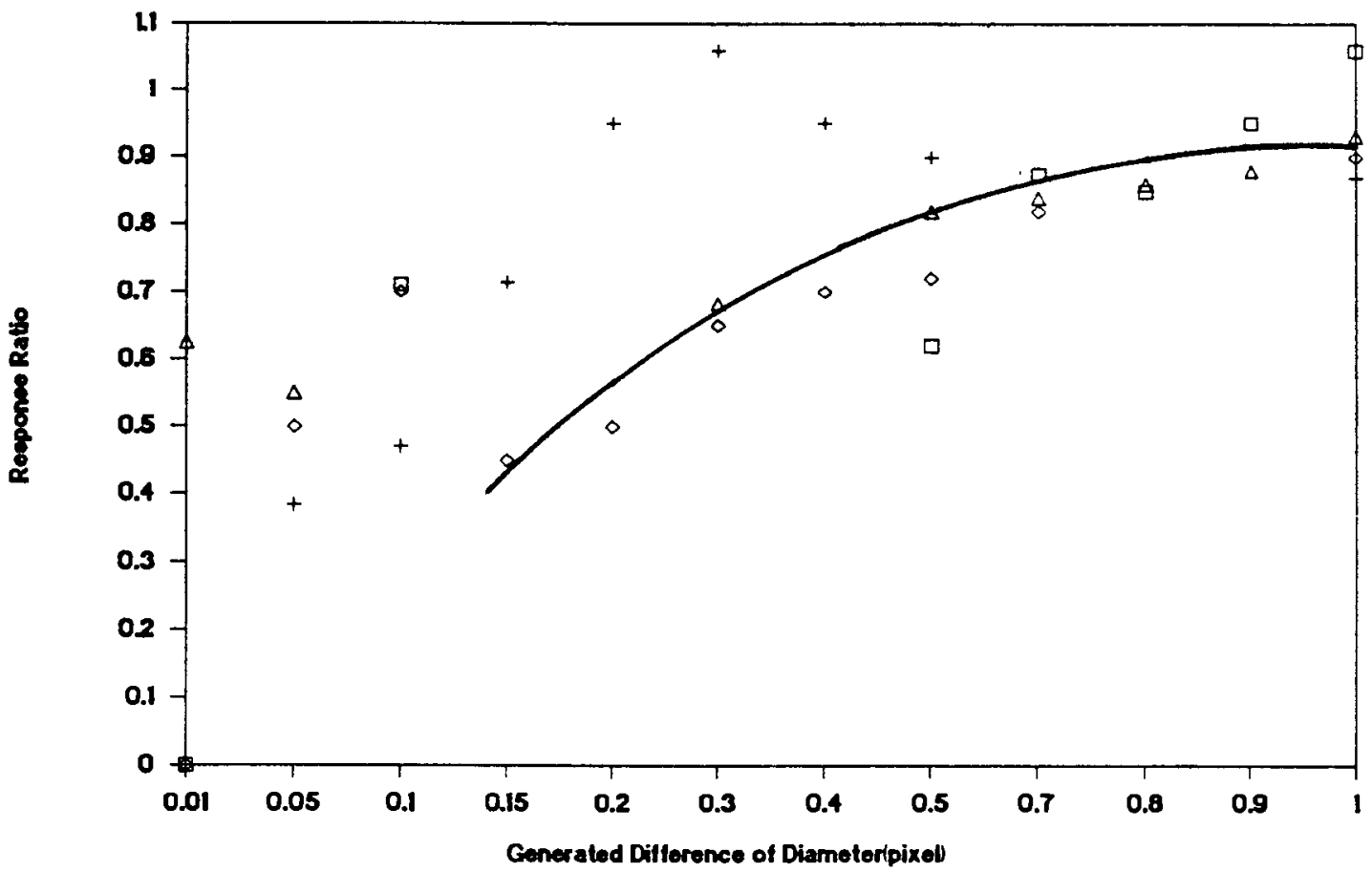

Figure 46. Resolution of circle in binary space for different diameters

To achieve knowledge about the accuracy of a vision system for dimensional inspection of circles, investigations have to be done in the resolution of the system and the use of subpixel accuracy. Then, the entire system must be tested, and an error model, including image analysis, calibration and resolution errors needs to be setup.

\subsection{RECOMMENDATIONS}

A system extension for measurements of objects with straight lines is appropriate. The detection process of straight line edges is similar to circular edges, but other approaches have to be found to obtain the geometrical parameters like distances between two edges. An interesting algorithm for the detection of straight line edges has been proposed by 
Petkovic, et al [12].

Another important issue in the area of vision systems and image processing is the development of standards to compare hardware and software. Edge detection algorithms have to be compared using the same standard images. This would make it easier to choose the right method for a certain application. The Abingdon Cross Benchmark test [25] for comparison of image processing hardware is one step towards the standardization of comparisons in the field of vision systems.

The accuracy of vision systems for inspection can also be increased by using higher magnification lenses combined with precision tables, or by the use of subpixel measures. Much significant improvements can be realized by using high resolution CCD elements. In April 1989 the first CCD chip with 2048 × 2048 elements was introduced [26]. However to make this technology applicable for the task of dimensional inspection, more powerful computers have to be built to process the image in a reasonable amount of time. 


\section{REFERENCES}

[1] F. Etesami, "Position Tolerance Verification Using Simulated Gaging", (to be published).

[2] J. Hollingum, "Machine Vision - The Eyes of Automation", Springer Verlag, 1984 .

[3] T.Y. Young, K.S. Fu, "Handbook of Pattern Recognition and Image Processing", Academic Press, 1986.

[4] H.-J. Warnecke and C.P. Keferstein, "Optoelectronic sensor system based on picture processing", Technische Messen tm, 52nd Jahrgang, $9 / 1985$.

[5] J.F. Bremner, "Automatic Vision Measurement System for the Inspection of Shapes Cut in Sheet Material", Second International Conference on Image Processing and its Applications, 1986.

[6] T.S. Huang, "Picture Processing and Digital Filtering", Springer Verlag, Berlin and New York, 1975.

[7] R.C. Gonzalez and P.A. Wintz, "Digital Image Processing", AddisonWesley, 1977.

[8] A. Rosenfeld and A.C. Kak, "Digital Picture Processing", Academic Press, New York, 1982.

[9] T. Peli and D. Malah, "A Study of Edge Detection Algorithms", Computer Graphics and Image Processing 20, pp. 1-21, 1982.

[10] L.J. Van Vliet and I.T. Young, "A Nonlinear Laplace Operator as Edge Detector in Noisy Images", Computer Vision, Graphics, and Image Processing 45, pp. 167-195, 1989.

[11] M. Rueff and K. Melchior, "Bildlib:' The Image Analysis Software at IPA," Journal of Robotic Systems, 2(2), pp. 179-198, 1985.

[12] D. Petkovic, W. Niblack, and M. Flicker, "Projection-based High Accuracy Measurement of Straight Line Edges", Machine Vision and Applications 1, pp. 183-199, 1988.

[13] "ITEX PCplus Programmer's Manual", Image Technology Incorporated, 1987.

[14] G.A. Baxes, "Digital Image Processing", Prentice Hall, Inc., Englewood Cliffs, New Jersey, 1984. 
[15] L.G. Roberts, "Machine perception of three dimensional solids", in Optical and Electro Optical Information Processing, pp. 159-197, M.I.T. Press, Cambridge, mass., 1965.

[16] J. Lee, "Digital Image Enhancement and Noise Filtering by Use of Local Statistics", IEEE Transactions on Pattern Analysis and Machine Intelligence, Vo1. PAMI-2, No. 2 March 1980.

[17] J. Lee, "Refined Filtering of Image Noise Using Local Statistics", Computer Graphics and Image Processing 15, pp. 380-389, 1981.

[18] G.S. Robinson, "Edge Detection by Compass Gradient Masks", Computer Graphics and Image Processing 6, pp. 492-501, 1977.

[19] G. Johannsen and J. Bille, "A Threshold Selection Method using Information Measure", Proc. International Conference on Pattern Recognition, Munich, 1982. IEEE Comp. Soc. Press, Silver Spring, $\mathrm{MD}$.

[20] S. Thomas and Y.T. Chan, "A Simple Approach for the Estimation of Circular Arc Center and Its Radius", Computer Vision, Graphics, and Image Processing 45, pp. 362-370, 1989.

[21] W.K. Pratt, "Digital Image Processing", Wiley, New York, 1977.

[22] Slama, "Manual of Photogrammetry", Fourth Edition, p. 1003, American Society of Photogrammetry, Virginia, 1980.

[23] R.Y. Tsai, "A Versatile Camera Calibration Technique for HighAccuracy 3D Machine Vision Metrology Using off-the-Shelf TV Cameras and Lenses", IEEE Journal of Robotics and Automation, Vol. RA-3, No. 4, August 1987.

[24] W.H. Press, B.P. Flannery, S.A. Teukolsky, and W.T. Vetterling, "Numerical Recipes: The Art of Scientific Computing", Cambridge University Press, Cambridge, 1986.

[25] K. Preston Jr., "The Abingdon Cross Benchmark Survey", Computers, Volume 22, No.7, 1989.

[26] VDI (Verein Deutscher Ingenieure) - Nachrichten, April 1989. 
APPENDIX A

THE PROGRAY SOURCE CODE OF

THE LOCAL STATISTIC METHOD 
\#include "c: $\backslash p c p l u s \backslash$ itex $\backslash$ itexpfg.h"

\#include "c: \pcplus \itex\stdtyp.h"

\#include <math.h>

$\operatorname{LOCALST}(x, y$, sigmal, bright)

int $x, y$, sigmal, *bright;

/* Refined Filtering of Image Noise Using Local Statistics

/* Paper Jong-Sen Lee/Computer Graphics and Image Processing 15

/* 380-389 (1981)

/* input: $x, y$-coordinates of center of $7 * 7$ matrix

/* sigmal-noise variance ( proposed 300 by Lee)

/* output: bright-noise filtered brightness value for pixel $\mathrm{x}, \mathrm{y}$ */

int matrix[4][4];

int $i, j, k, 1$, add, sub, or, submean;

int $i \mathrm{i}=0, \mathrm{j} j=0$;

float mean, var, kfactor;

float sum_mean=0., sum_var $=0$.;

for $(i=x-2 ; i<-x+2 ; i=i+2)($

iit+;

$\mathbf{j} \mathbf{j}=\mathbf{0}$;

for $(j=y-2 ; j<=y+2 ; j=j+2)($

$j \mathrm{j}+$;

$/ *$ call submatr 3 subroutine - which calculates the submean */

$/ *$ of the moving $3 * 3$ window */

$\operatorname{SUBMATR} 3(\mathbf{i}, \mathbf{j}$, \&submean) ;

$/^{*}$ generation of submatrix $3 * 3-\operatorname{matrix}[x=1 \ldots x=3][y=1 \ldots y=3] * /$ $\operatorname{matrix}[i 1][j j]=$ submean; $)\}$

/* call edge_or subroutine - to find the orientation of the edge */

/* in matrix $3 * 3$

/* for orientation index see subroutine edge_or

EDGE_OR(matrix, \&or);

/* pattern calculation of mean and variance

/* ATTENTION index for orientation different as proposed by Lee */ switch (or) 1

case 0: for $(\mathrm{k}=\mathrm{x}-3 ; \mathrm{k}<=\mathrm{x}+3 ; \mathrm{k}++)$ l

for $(1=\mathrm{y}-3 ; 1<\mathrm{y} ; 1++)$

sum_mean=sum mean $+\operatorname{rpixel}(k, 1) ;\}$

mean = sum mean $/ \overline{2} 8$.;

for $(\mathrm{k}=\mathrm{x}-\overline{3} ; \mathrm{k}<-\mathrm{x}+3 ; \mathrm{k}++) \mathrm{l}$

for $(1=\mathrm{y}-3 ; 1<=\mathrm{y} ; 1++)$

sum_var $=$ sum_var $+\operatorname{pow}((\operatorname{rpixel}(k, 1)-$ mean $), 2.0)$; 


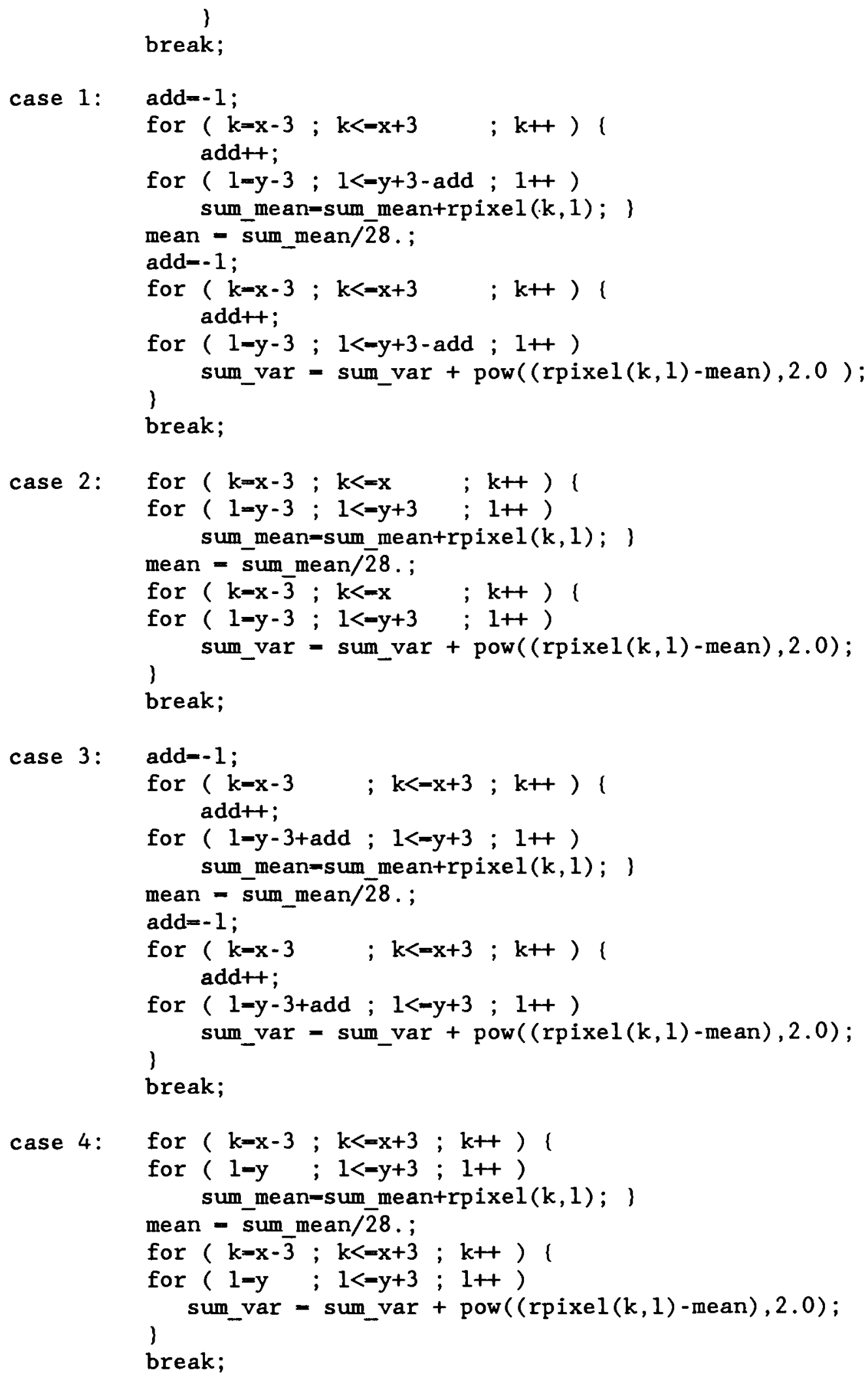




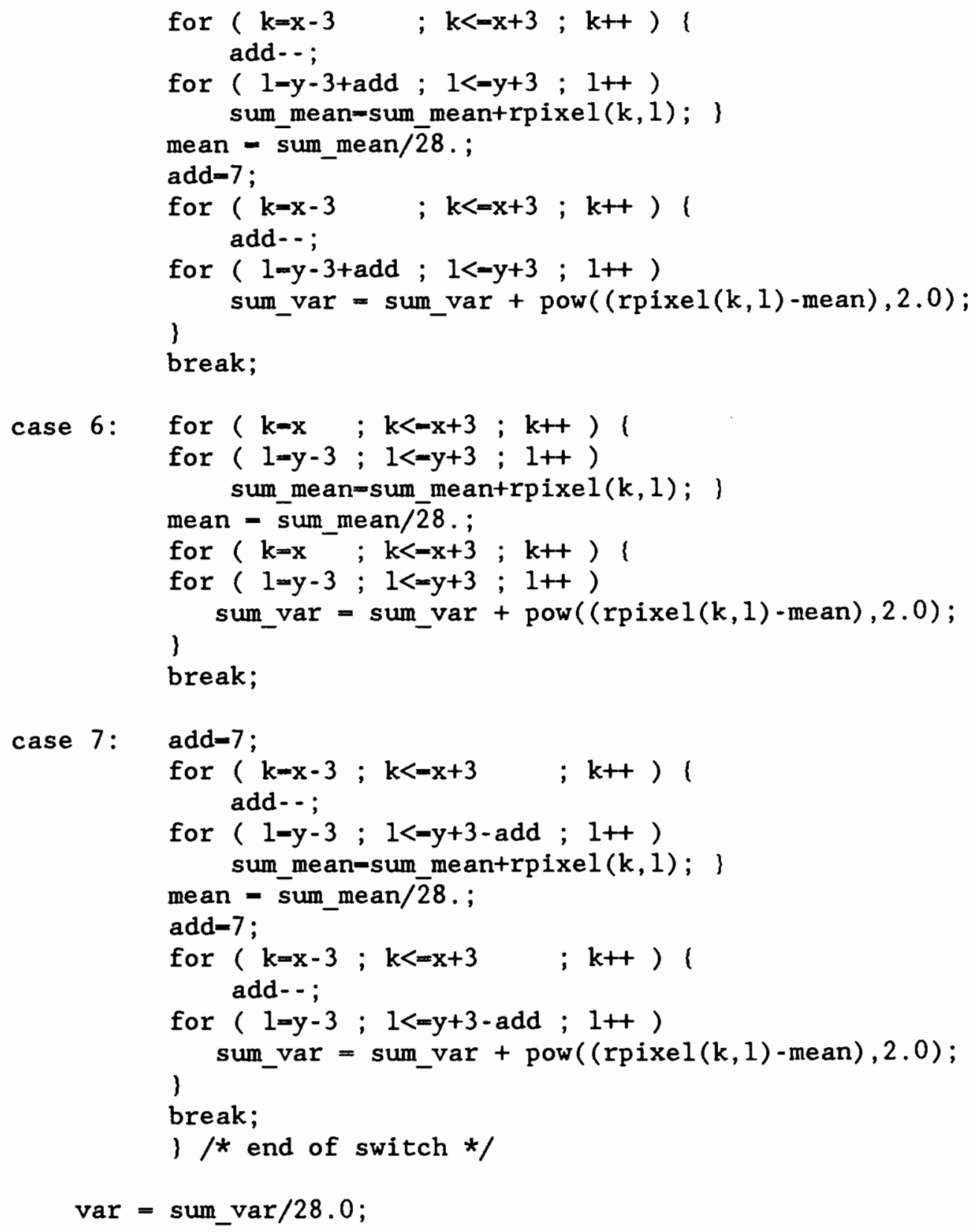


$\operatorname{SUBMATR} 3(\mathrm{x}, \mathrm{y}$, submean)

int $x, y, *$ submean;

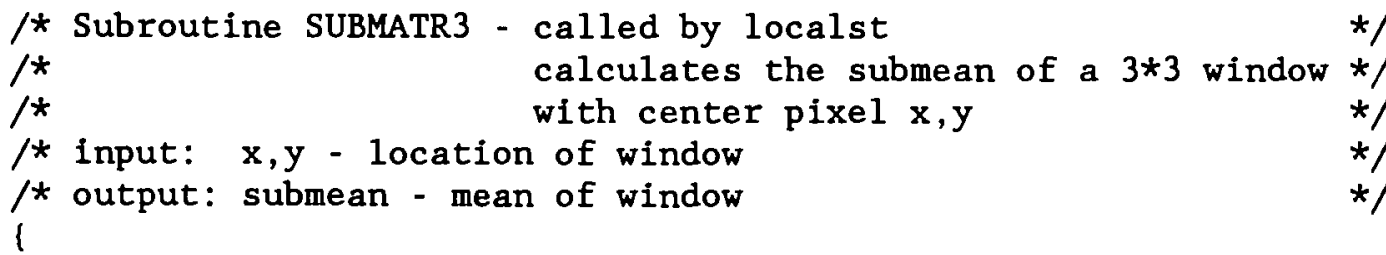

int $1, j$;

float sum;

sum $=0$;

for $(i=x-1 ; i<=x+1 ; i++)($

for $(j=y-1 ; j<-y+1 ; j++)$ sum $=\operatorname{sum}+\operatorname{rpixel}(i, j) ; 1$

*submean $=(\operatorname{sum} / 9)+.0.5 ; / *+0.5$ to simulate $\operatorname{ROUND}(\mathrm{x}) * /$

) $\quad / *$ end of subroutine SUBMATR $3 * /$

EDGE_OR(matrix,or)

int matrix[][4];

int *or;

/* Subroutine EDGE_OR - called by localst */

/* calculates edge orientation within a */

* $3 * 3$ matrix */

/* edge_or uses Three-level Simple Masks as proposed by Lee */

/* Paper Edge Detection by Compass Gradient Masks */

/* Guener S. Robinson / Computer Graphics and Image Processing */

/* $6,492-501(1977) \quad * /$

/* input: matrix $3 * 3$ */

/* output: orientation - index as in paper of Robinson */

$1 / *$ start of subroutine EDGE_OR * $)$

int $\operatorname{grad}[3]$, stack, istack, $\mathrm{i}$;

/* Index 0 - Gradient NORTH $\operatorname{grad}[0]=\operatorname{matrix}[1][1]+\operatorname{matrix}[2][1]+\operatorname{matrix}[3][1]$ - matrix[1][3] - matrix[2][3] - matrix[3][3]; stack $=\operatorname{grad}[0]$; istack $=0$;

Index 1 - Gradient NORTHWEST $\operatorname{grad}[1]=\operatorname{matrix}[1][1]+\operatorname{matrix}[2][1]+\operatorname{matrix}[1][2]$

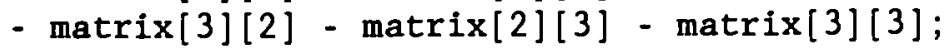




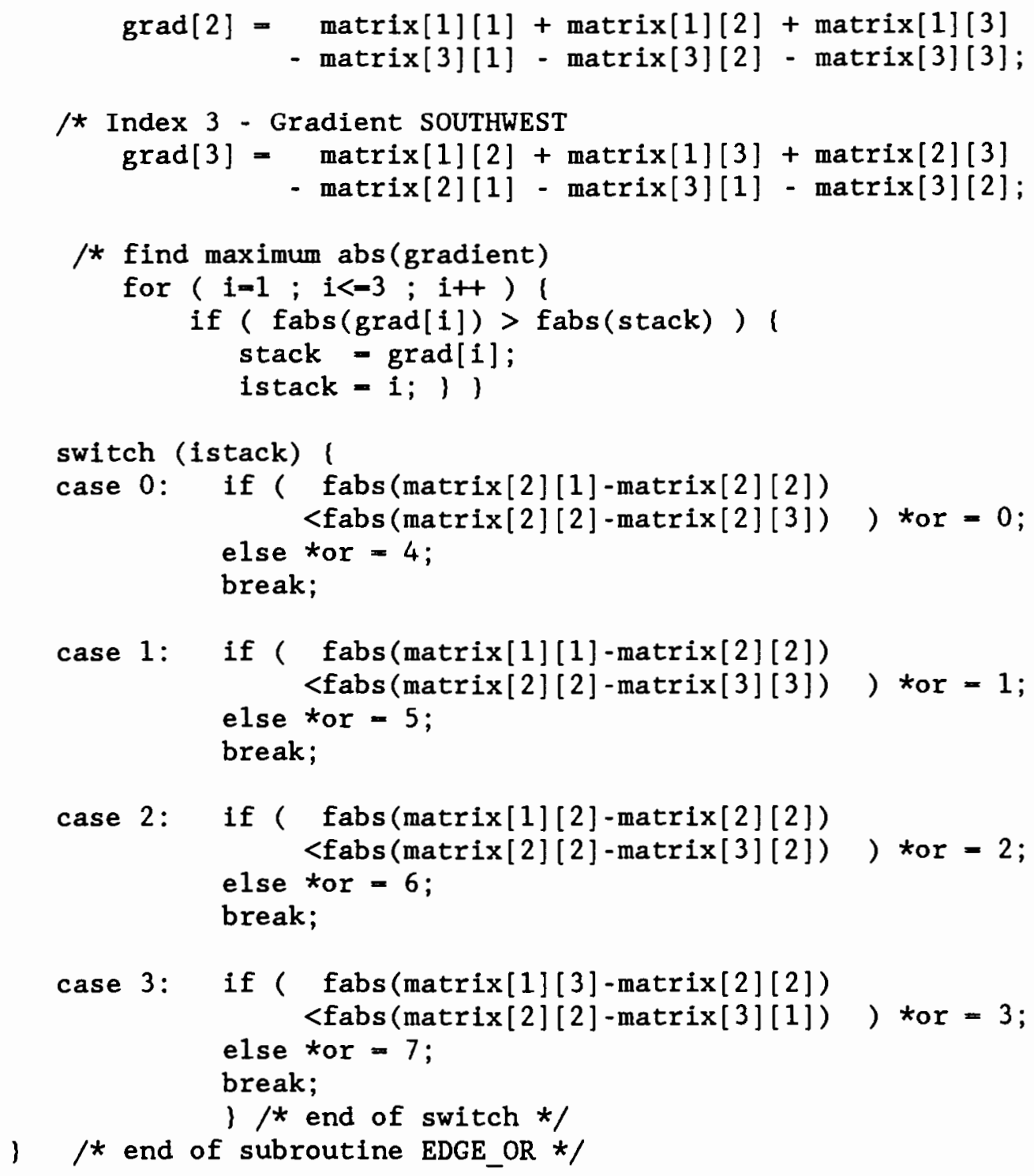


APPENDIX B

THE PROGRAM SOURCE CODE OF

THE CONTOUR FOLLOWING ALGORITHM 
\#include "c:\pcplus \itex \itexpfg.h"

\#include "c: \pcplus \itex\stdtyp.h"

\#include <stdio.h $\rangle$

\#define OBJECTIN 0

\#define BACKINT 255

BTRACE ( $x, d x, y, d y$, display1, display2, xbuff, ybuff, buffmax, bufflgth, berr)

int *xbuff, *ybuff, *bufflgth, *berr;

int $\mathrm{x}, \mathrm{dx}, \mathrm{y}, \mathrm{dy}, \mathrm{display} 1$, display2, buffmax;

/* Boundary Tracing Algorithm - Extract Boundary Pixels */

/* BTRACE of Binary Images */

/* INPUT $\mathrm{x}, \mathrm{dx}, \mathrm{y}, \mathrm{dy}$ : object window coordinates */

/* displayl : 1 - boundary pixels are displayed */

/* display2 : 1 - boundary pixels coordinates */

/* are written to file WBUFF.DAT */

/* buffmax : max. array length */

$/ *$ OUTPUT xbuff : boundary coordinates array with */

$/ *$ ybuff : length $\langle$ buffmax $\rangle$, to be defined */

$/ *$ in calling program */

$/ *$ bufflgth : length of array xbuff,ybuff */

$/ * \quad$ ATTENTION array [ 0 . bufflgth ] */

/* \# of boundary pixels is bufflgth+1 */

$(/ *$ start of BTRACE */

int $x n, y n, x i n, y i n, i, b u f f l, e r r$;

*berr $=0$;

$\mathrm{xn}=\mathrm{x}$;

$\mathrm{yn}=\mathrm{y}$;

start: SEARCHINIT ( $x n, x+d x, y n, y+d y, \& x i n, \& y i n)$;

xbuff $[0]=x i n$;

ybuff $[0]=y$ in;

$i=0$;

NEXTBO (xbuff, ybuff, i, \&err);

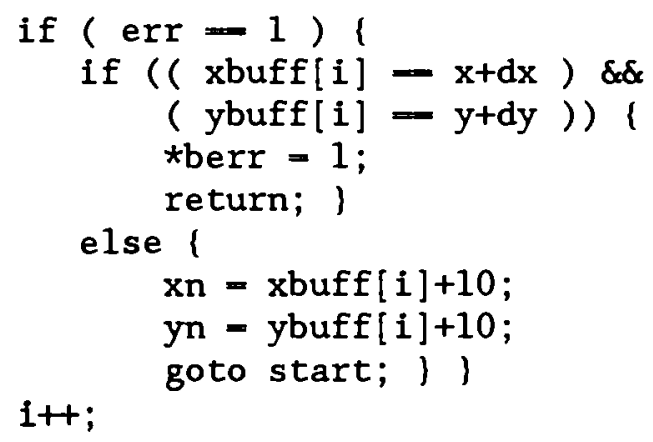




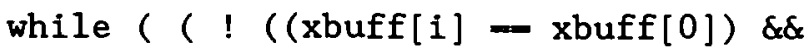

$($ ybuff $[i]-\operatorname{ybuff}[0]))) \& \&(i<\operatorname{buffmax}))($

NEXTBO( xbuff, ybuff, $\mathbf{i}$, \&err);

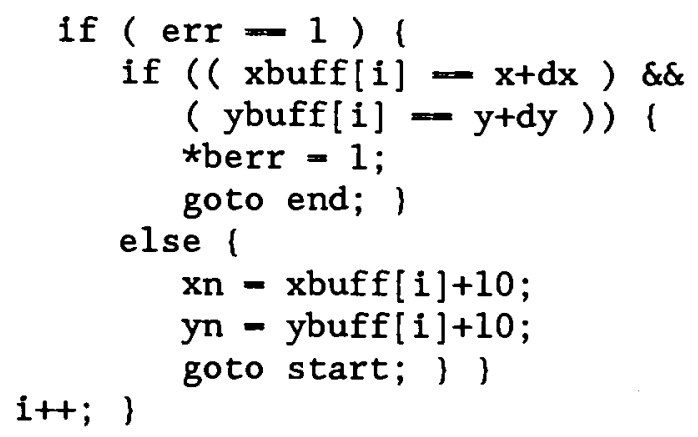

int countx, county;

$* x$ in $=-1$
*yin $=-1$

for ( count $x=x 1 ;$ count $x<=x 2 ;$ countxt+ ) 1 for ( county-y1 ; county<-y2 ; county++ ) ( if ( (rpixel (countx, county) - OBJECTIN) \&\& ( doe (countx, county) $<4$ ) $($ doe (countx, county) $>0))($ *xin $=$ countx;

*yin = county; 


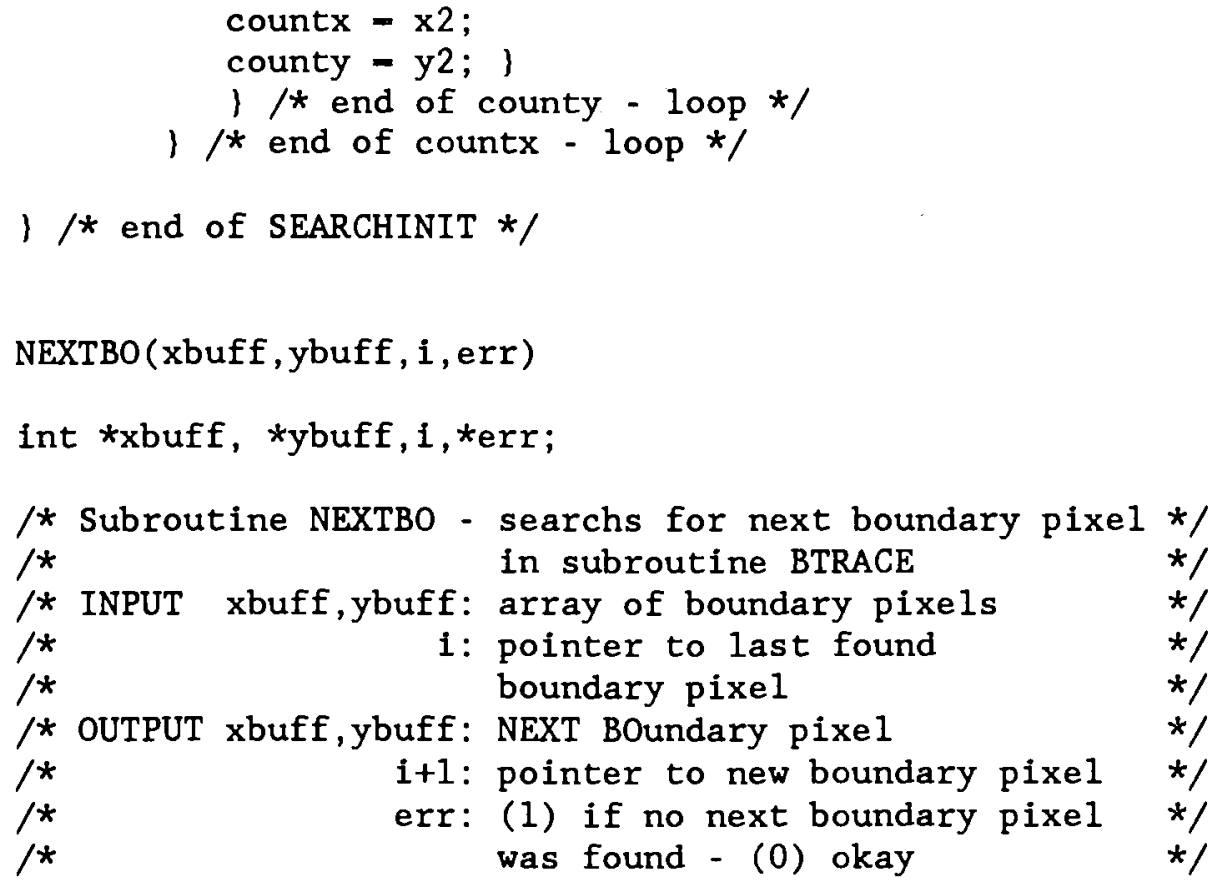




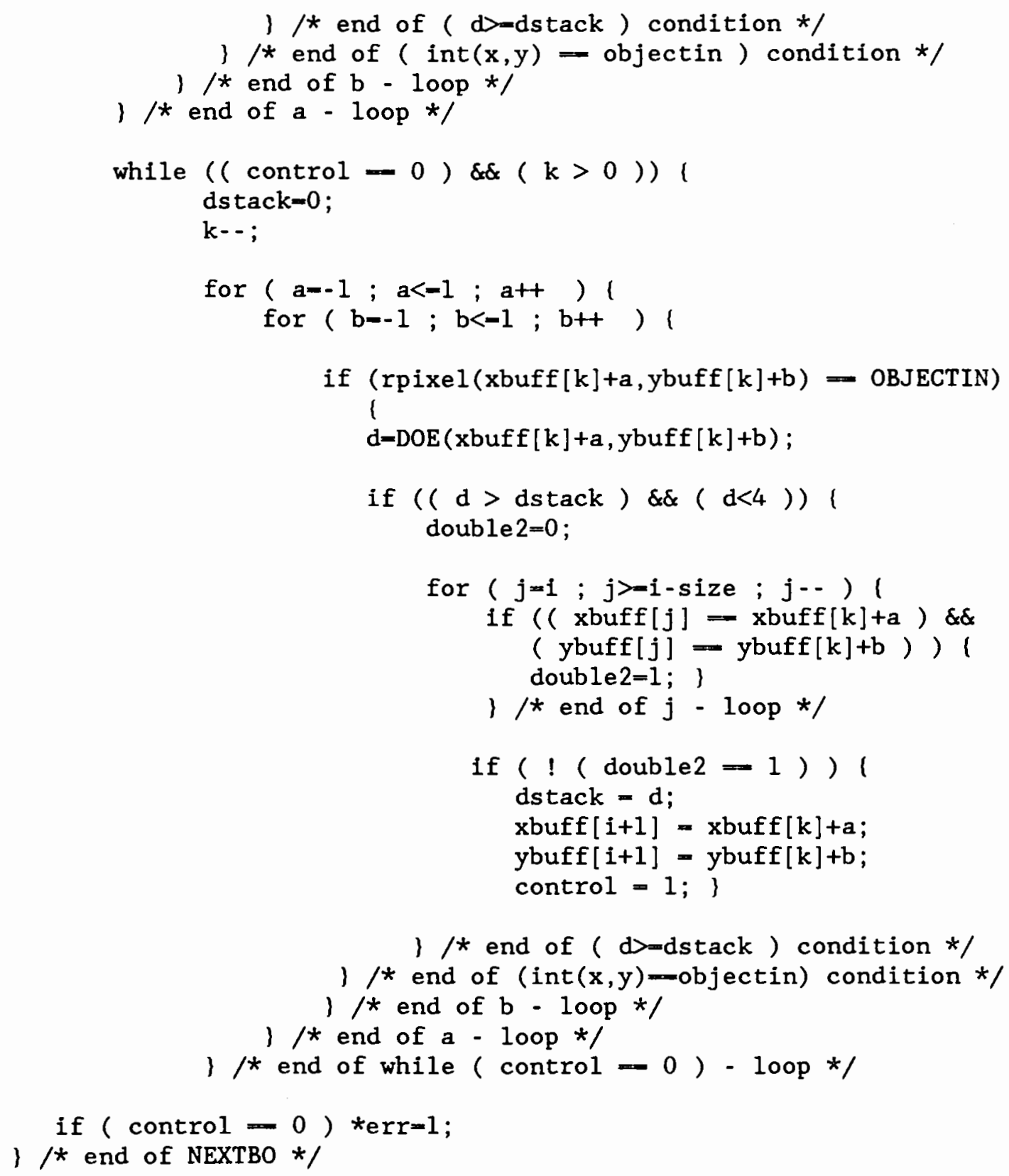

$\operatorname{DOE}(x, y)$

int $x, y$;

/* Subroutine DOE - calculates the degree of */

/* exposure of pixel $x, y$ */

1

int $\mathrm{d}=0$;

if $(\operatorname{rpixel}(x+1, y)=$ BACKINT $) d++$; 


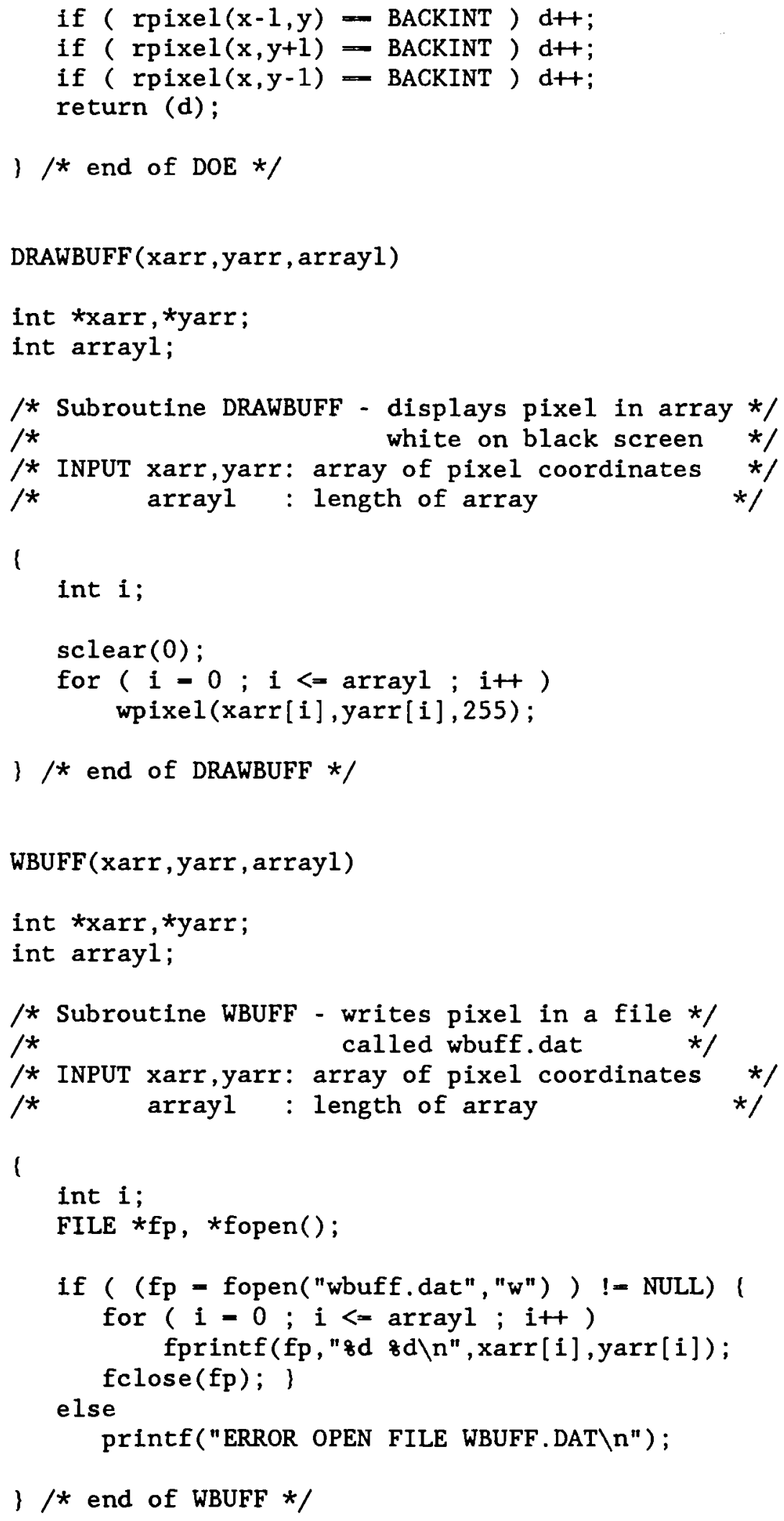




\section{APPENDIX C}

THE PROGRAY SOURCE CODE OF

THE AUTOMATED THRESHOLD SELECTION ALGORITHM 
\#include "c:\pcplus \itex \itexpfg.h"

\#include "c:\pcplus \itex\stdtyp.h"

\#include <math.h〉

\#include <stdio.h $\mathrm{h}$

\#include $\langle$ graph.h $\rangle$

long HISTVALS [255];

float $\mathrm{P}[255], \mathrm{H}[255], \mathrm{H} \_[255], \mathrm{S}[255], \mathrm{S}_{-}[255], \mathrm{DELTA}[255]$;

$\operatorname{THSELECT}(\mathrm{x}, \mathrm{dx}, \mathrm{y}, \mathrm{dy}$, ints, inte, display, thval)

int $x, d x, y, d y$, ints, inte, display;

int *thval;

/* Subroutine THSELECT - Automatic Threshold Selection */

/* using the Entropy of the Gray */

/* Leve1 Histogram */

/* THSELECT is based on the algorithm proposed by */

/* G.Johannsen, J.Bille IEEE CH1801 1982, A Threshold */

/* Selection Method using Information Measures */

/* INPUT $\mathrm{x}, \mathrm{dx}, \mathrm{y}, \mathrm{dy}$ : window-coordinates of operation */

$/ x$

ints: intensity to start evaluation */

$/ *$

inte: intensity to end evaluation

/* display: 0 - evaluation is not displayed

$/ *$

/* OUTPUT thval: selected threshold

1 - evaluation is displayed

*/

$* /$

*1

*1

float LK, LK_, LKSUB1, LKSUB1_;

float STACK;

float NFLOAT, HISTFL;

int STACKTH;

int $i, j, k, 11,12$;

int $i \mathrm{i}=0, \mathrm{ksum}=0$;

long $\mathrm{N}$;

char cl;

/* Generation of Histogram */

histogram ( $x, y, d x, d y, 1,1,0, \operatorname{HISTVALS})$;

$\mathrm{N}=0 ; \operatorname{STACK}=1$;

for $(i=0 ; i<=255 ; i++) N=N+H I S T V A L S[i]$;

for $(j=0 ; j<-255 ; j+)$ (

NFLOAT-N;

HISTFL-HISTVALS $[j]$;

$P[j]=$ HISTFL/NFLOAT; $]$

$\mathrm{H}[-1]=0 ; \mathrm{H}_{-}[-1]=0$;

$\mathrm{LK}=0$;

if ( display -1 ) I

clearscreen(_GCLEARSCREEN);

printf("INTENSITY K DELTA[k] $\mathrm{K} \backslash \mathrm{n}$ "); ) 


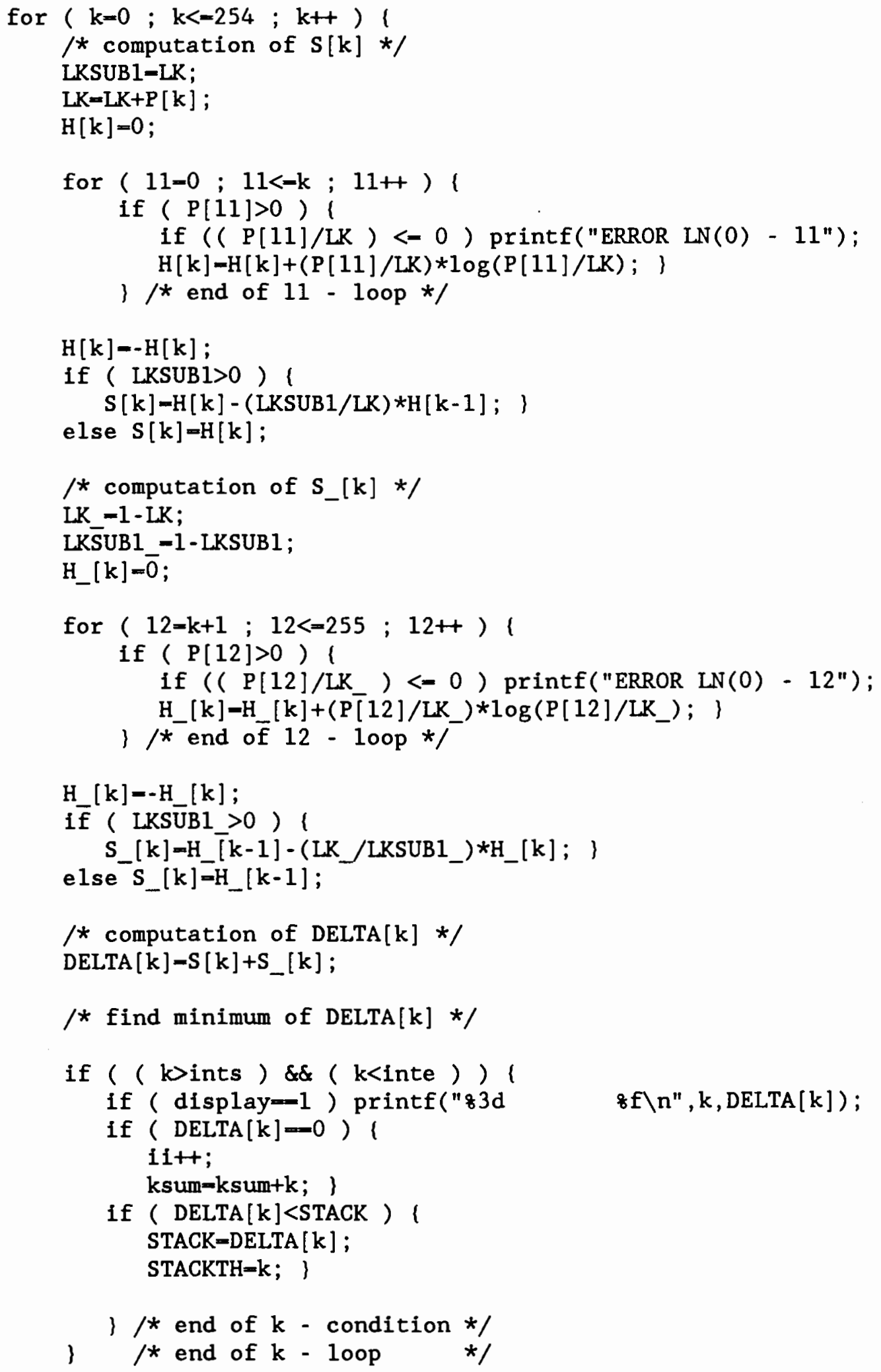


if ( $i$ i $>-3$ ) STACKTH=ksum/ii;

*thval-STACKTH;

if (display - 1) ( clearscreen(_GCLEARSCREEN);

printf("\nTHRESHOLD: \&d \n",STACKTH);

printf(" $\backslash n D E L T A$ MIN: \&f $\backslash n \backslash n \backslash n \backslash n "$, STACK);

printf("Press key to continue $\backslash \mathrm{n}^{\prime}$ );

cl-getchar();

cl-getchar();

_clearscreen(_GCLEARSCREEN); \}

) $/$ * end of THSELECT * / 
APPENDIX D

THE PROGRAM SOURCE CODE OF

THE RADIUS ESTIMATION ALGORITHM 
\#include <math.h>

ARCESTIM(xarr, yarr, N, sigma, centerx, centery, radius, biasR)

int $\operatorname{xarr}[], y \operatorname{arr}[]$;

int $\mathrm{N}$;

float sigma;

float *center,$*$ centery,$*$ radius,$*$ bias ;

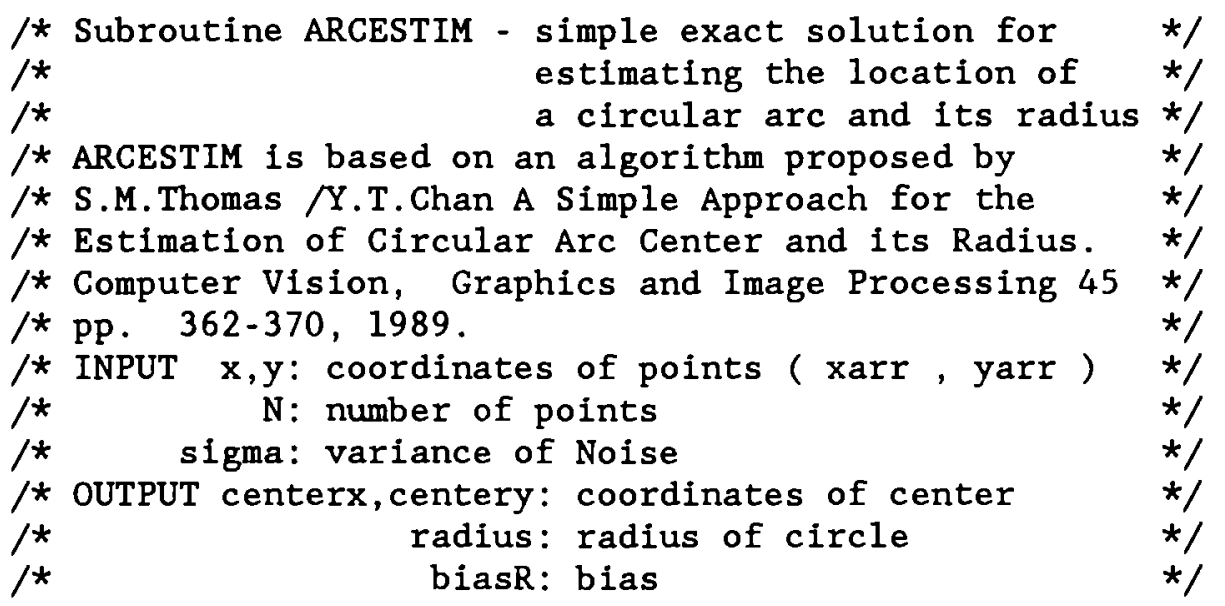

int $i$;

float sum $x=0$, sumy $=0$, sumxy $=0$, sumx $2 y=0$;

float $\operatorname{sum} x 2=0$, sumx $3=0$, sumxy $2=0$, sumy $2=0$, sumy $3=0$;

float a1, a2, b1,b2, c1, c2;

float xavg, yavg, R, sigma2, bxavg, byavg, br2;

for $(i=0 ; i<-N-1 ; i++)($

sumx-sumx+xarr [1];

sumy=sumy+yarr [i];

sumxy-sumxy+(float) $(x \operatorname{arr}[i]) *(f l o a t)(y \operatorname{arr}[i])$;

$\operatorname{sum} x 2 y=\operatorname{sumx} 2 y+($ float $)(x \operatorname{arr}[i]) *(f l o a t)(\operatorname{xarr}[i])$ *(float) (yarr [i]);

sumx2-sumx $2+($ float $)(x a r r[i]) *($ float $)(\operatorname{xarr}[i])$;

sumx 3-sumx 3+(float) (xarr [i] )*(float) (xarr [i]) *(float) (xarr $[i])$;

$\operatorname{sumxy2}=\operatorname{sumxy} 2+($ float $)(\operatorname{xarr}[i]) *($ float $)(y \operatorname{arr}[i])$ *(float) (yarr $[i])$;

sumy $2=$ sumy $2+($ float $)(y \operatorname{arr}[i]) *($ float $)$ (yarr [i]) ; sumy3-sumy $3+($ float $)$ (yarr $[i]) *($ float $)$ (yarr $[\mathbf{i}])$ *(float) (yarr $[\mathbf{i}])$; )

al $-2 *(\operatorname{sum} x * \operatorname{sum} x-N * \operatorname{sum} x 2)$;

a2 $=2 *$ ( sum $x *$ sumy $-N *$ sumxy);

$\mathrm{b} 1=\mathrm{a} 2$;

b2 $=2 *$ ( sumy* sumy $-\mathrm{N} *$ sumy 2$)$;

c1-sum $x 2 *$ sum $x-N * \operatorname{sum} x 3+\operatorname{sum} x *$ sumy $2-N * \operatorname{sum} x y 2$;

c2-sumx $2 *$ sumy $-N *$ sumy $3+$ sumy*sumy $2-N * \operatorname{sum} x 2 y$; 


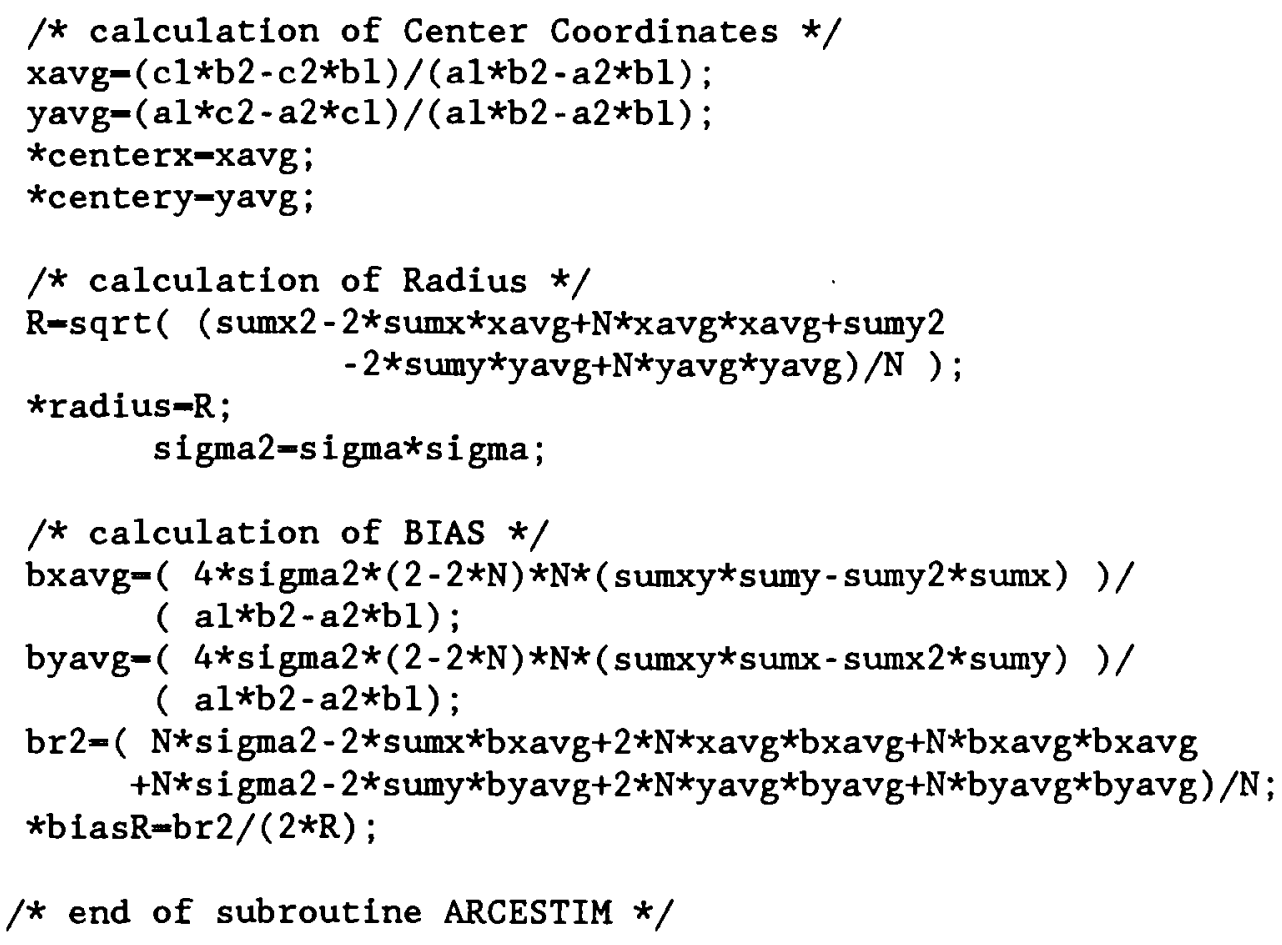


APPENDIX E

THE PROGRAM SOURCE CODE OF THE IMAGE GENERATOR 


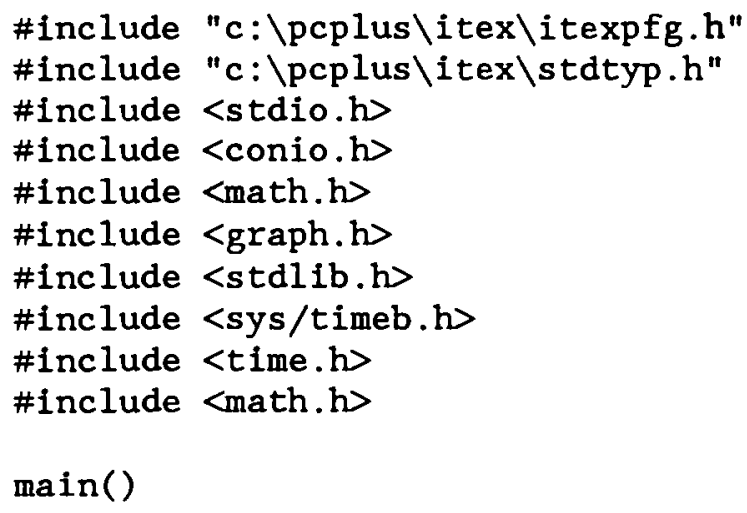

/* Program IMAG_GEN

/* Circular Image Generation with Smoothing,

$/ *$ System Noise Simulation and Object Noise ( Texture) */

/* Simulation

$(/ *$ start of IMAG_GEN *)

int stop, radius, center_x, center_y, intensity_p, background;

int $x w 1, x w 2, y w 1, y w 2, r a \bar{d}$, new_intensity, $i, j$;

int rang_e1, rang_e2,pixel_num,diff,old_intensity;

int kbkit(void), cnt,es;

long $\mathrm{xcl}$, ycl;

unsigned rand_seed;

double rad2, $\mathrm{xc} 2, \mathrm{yc} 2, \mathrm{zc} 2$, rnd2, rnd21;

float noise, mean_n,std_n, range_n, rand4;

char $\mathrm{cl}$;

FILE *fp, *fopen () ;

_clearscreen(_GCLEARSCREEN);

INITSYS();

printf("Program - IMAG_GEN $\backslash n \backslash n$ ");

printf("Image Simulation for Circular Features. $\backslash n \backslash n$ ");

printf("Simulation Operations: - Smoothing $\backslash n$ ");

printf ("

printf("

- Additive System Noise $\backslash n^{\prime \prime)}$;

printf("Circle Generator $\backslash n \backslash n ")$;

- Texture Simulation $\left.\backslash n \backslash n \backslash n^{\prime \prime}\right)$;

printf("Coordinates of the center of the circle $\backslash n$ ");

printf("X: ");

$\operatorname{scanf}($ " $z$ " , \&center_x);

printf("Y: ");

scanf (" $z d "$ " dcenter_y);

printf("\n\nRadius of the circle R: ");

scanf (" $z \mathrm{~d} "$, \&radius) ;

printf(" $\backslash n \backslash n I n t e n s i t y$ of the pixels INSIDE the circle: "); scanf (" od" , \&intensity_p);

printf("\nIntensity of BACKGROUND pixels: ");

scanf (" od", \&background); 


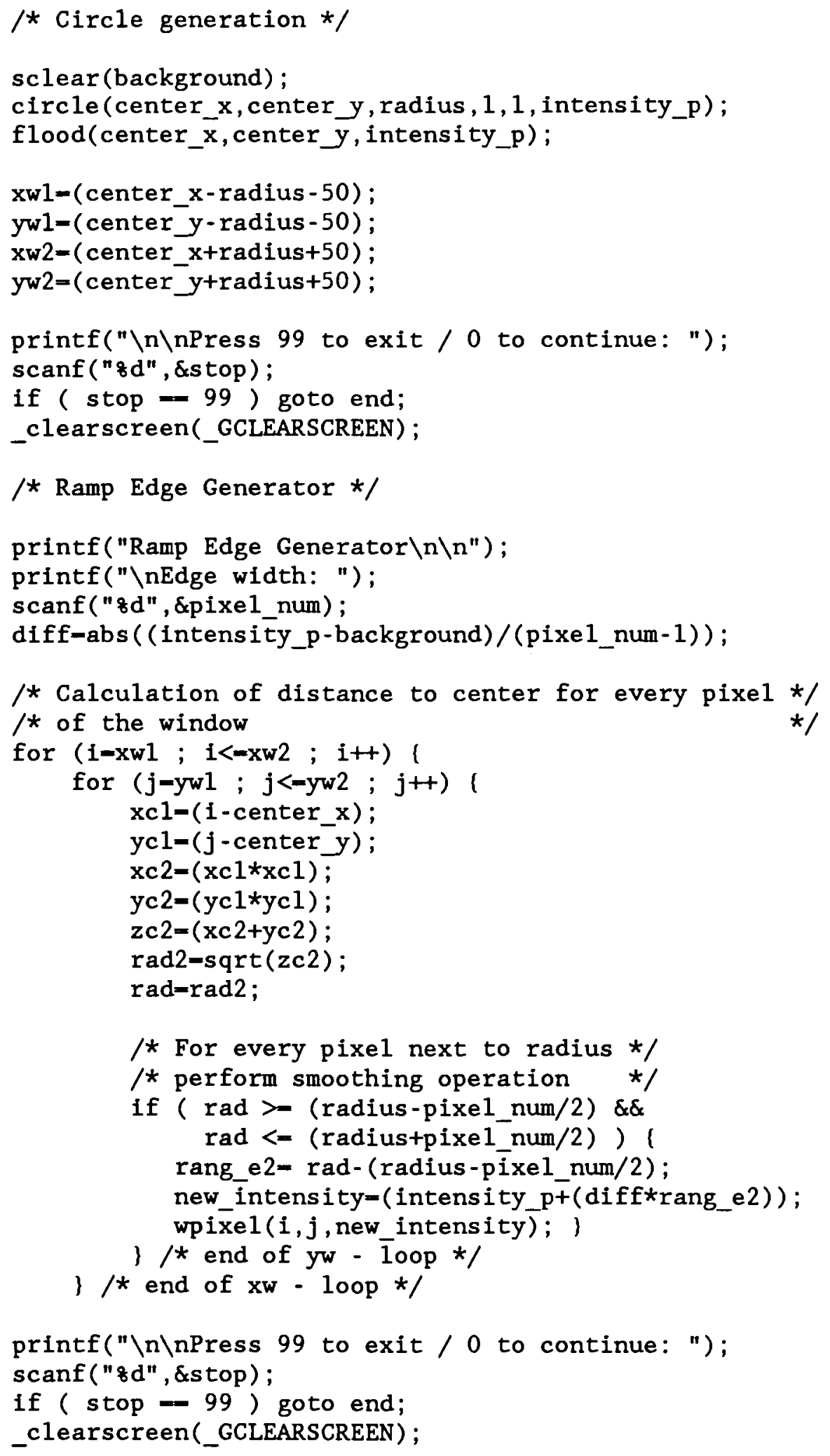




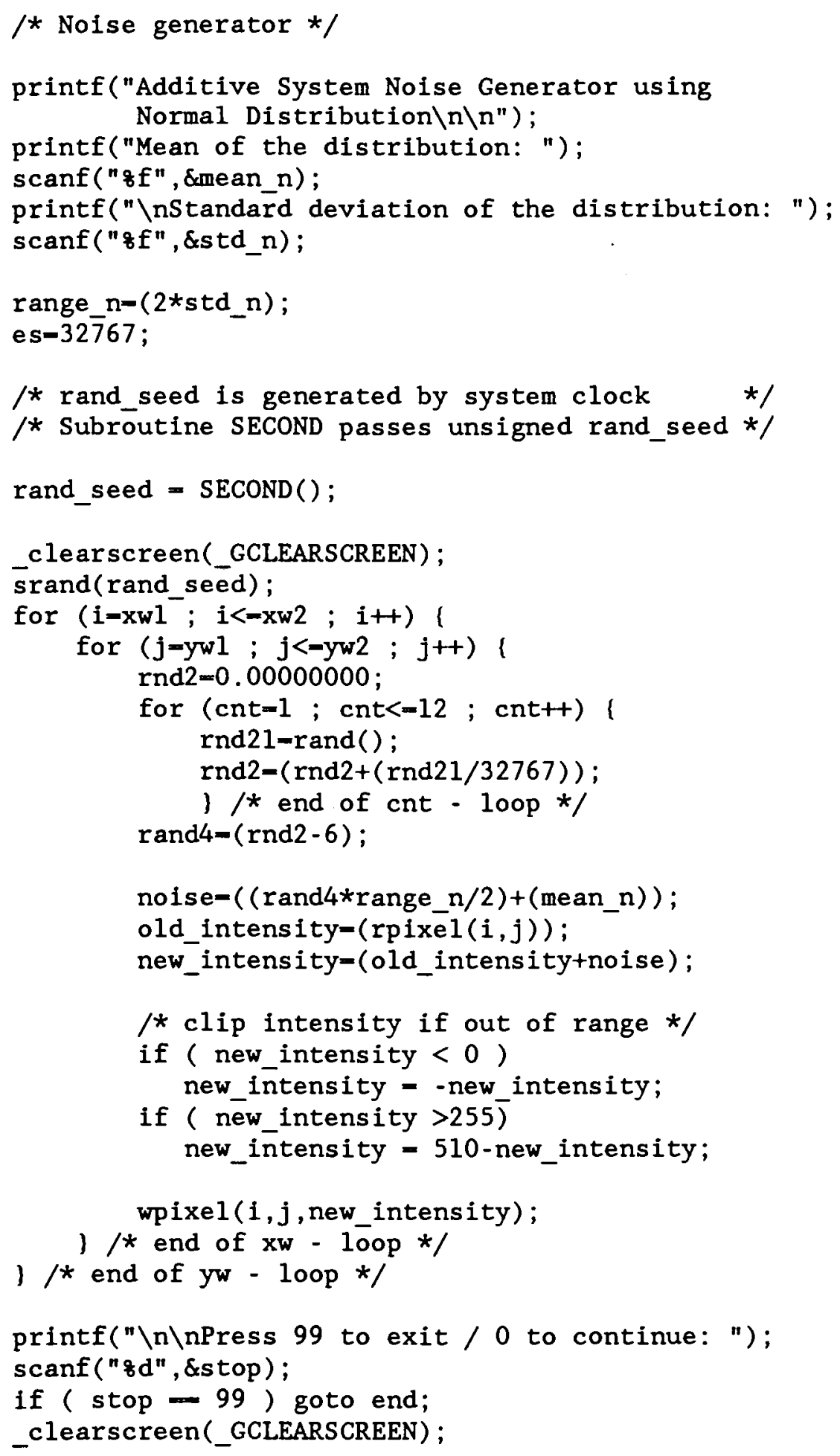


/* Texture Simulation */

printf("Object Texture Simulation $\backslash n \backslash n$ ");

printf("Using Additive Normal Distributed Noise in Object area $\left.\backslash n \backslash n^{\prime \prime}\right)$;

printf("Mean of the distribution: ");

scanf ("zf", \&mean $n$ );

printf("\nStandard deviation of the distribution: ");

$\operatorname{scanf}("$ of $f$ " , \&std_n) ;

range_n-(2*std_n);

es $=32 \overline{7} 67$;

rand_seed $=\operatorname{SECOND}()$;

_clearscreen(_GCLEARSCREEN);

srand (rand_seed);

for $(i=x w 1-i<=x w 2 ; i++)\{$

for $(j=y w 1 ; j<-y w 2 ; j++)($

xcl=(i-center_x $)$;

ycl- $(j$-center $y)$;

$\mathrm{xc} 2=(\mathrm{xc1} \times \mathrm{xc} 1)$;

yc $2=($ yc1*yc1);

$\mathrm{zc} 2=(\mathrm{xc} 2+\mathrm{yc} 2)$;

$\operatorname{rad} 2=\operatorname{sqrt}(\mathrm{zc} 2)$;

rad=rad2;

/* For every pixel within radius */

/* perform texture simulation */

if ( $\mathrm{rad}<$ radius ) 1

rnd $2=0.00000000$;

for $(\mathrm{cnt}=1 ; \mathrm{cnt}<=12 ; \mathrm{cnt} t)$ (

rnd21-rand ();

rnd2-(rnd2+(rnd21/32767) $)$;

) $/ *$ end of cnt - loop */

rand4-(rnd2-6);

noise $=(($ rand $4 *$ range_n/2) $+($ mean_n $))$;

old_intensity $=(\operatorname{rpixel}(i, j))$;

new_intensity=(old_intensity+noise);

wpixel ( $i, j$, new_intensity); )

) $/ *$ end of yw - Ioop */

$1 / *$ end of $x w-$ loop $* /$

clearscreen(_GCLEARSCREEN);

end: saveim(0,0,640,480,0, "SIMULATE. IMG" , " );

if ( $(f p-$ fopen ("prn", "wn) ) ! - NULL ) !

fprintf(fp, "NOISE \&f\n",std_n);

fclose (fp);

) else printf(" File couldn't be opened $\left.\backslash n^{\prime \prime}\right)$;

) $/ *$ end of IMAG_GEN */ 


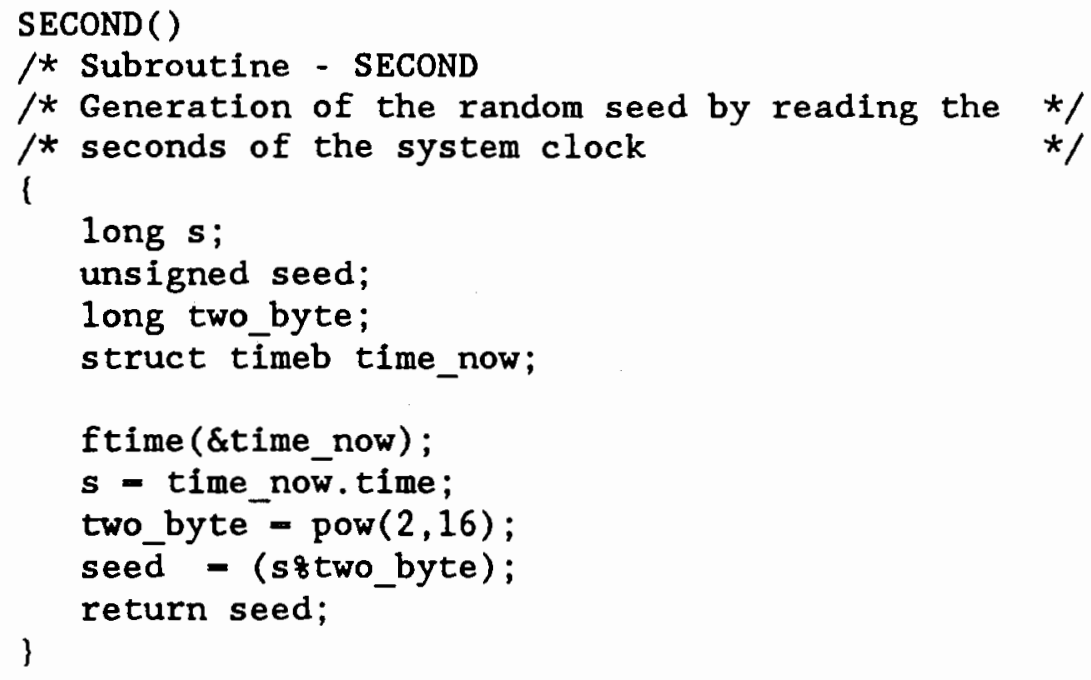


APPENDIX F

THE PROGRAY SOURCE CODE OF THE CALIBRATION ALGORITHM 
/* CALIBRATION of Vision Systems

/* Method of Roger Y. Tsai

/* Paper - A Versatile Camera Calibration

/* Technique for High-Accuracy 3D Machine Vision

/* Metrology Using Off-the-Shelf TV Cameras and

* Lenses, IEEE Journal of Robotics and Automation

$/ *$ Vol.RA-3, No.4, August 1987

\#include <stdio.h>

\#include <math.h>

\#include "c: $\backslash n r c p \backslash n r . h "$

\#include "c: $\backslash$ nrcp \nrutil.h"

/* global variables definition */

float d_x, Tx;

float $r \overline{4}, r 5, r 6, r 7, r 8, r 9$;

float $\star_{\mathrm{xf}}, \star_{\mathrm{yf}}, \star_{\mathrm{xw}}, \star_{\mathrm{yw}}, \star_{\mathrm{zw}}$;

int pnum;

$/ *$ constants of camera and vision system */

\#define DX $0.669291 \mathrm{e}-3 / *$ inch */ $/ * 17$ um $1 * 1 \mathrm{e}-6 \mathrm{~m} * /$

\#define DY $0.511811 \mathrm{e}-3 / *$ inch $* / / * 13$ um $1 * 1 \mathrm{e}-6 \mathrm{~m} * /$

\#define NCX 510

\#define NCY 492

\#define NFX 640

/* center pixel coordinates of frame memory */

\#define CX 320

\#define CY 240

/* uncertainty factor of sampling */

\#define SX 1.042

/ define free vector allocation size */

\#define MMP 50

\#define NNP 20

SGN (arg)

/* Subroutine SGN determines the sign of a float variable */

$/ *$ and returns 1 if $\operatorname{var}>-0$ otherwise 0 is returned */

float arg;

l

if $(\mathrm{fabs}(\arg ) \star \arg /(\arg * \arg )>0$ ) return 1 ;

else return 0 ;

1 
void CALIB ( $x f, y f, x w, y w, z w, p n u m)$

float $* x f, * y f, * x w, * y w, * z w ;$

int pnum;

/* Subroutine CALIBRATION */

* INPUT $\mathrm{xf}, \mathrm{yf}$ : screen or frame buffer coordinates */

/* $\quad \mathrm{xw}, \mathrm{yw}, \mathrm{zw}$ : world coordinates of calibration pattern */

/* pnum : number of calibration points */

/* OUTPUT file CALIB.DAT with calibration parameters */

$(/ *$ start of CALIB *)

float $* X d, * Y d, * * a a, * b b, * x x, * y y, * w w ;$

float dy;

float Ty_1r1,Ty_1r2,Ty_1r4,Ty_1r5,Ty_1Tx;

float $r_{-} \overline{1}, r_{-} 2, r_{-} 4, r_{-} 5, r_{i}, r_{j}$;

float $s \bar{r}, T 2 \bar{y}, \mathrm{Ty}$;

float $\mathrm{r} 1, \mathrm{r} 2, \mathrm{r} 3$;

float $T z, f, k l$, Tzopt, fopt, klopt;

float distx, disty;

float Xddist, Yddist;

float Xfdist, Yfdist;

float Xwdist, Ywdist, Zwdist;

int $i, n, e r r l, s$;

FILE $* \mathrm{fp}$, *fopen () ;

Xd - vector(1,MMP); / Numerical Recipes Subroutines */

Yd $=\operatorname{vector}(1$, MMP); $/ *$ vector, matrix

aa $=\operatorname{matrix}(1$, MMP, 1, NNP);

bb - vector $(1$, MMP);

$\mathbf{x x}=\operatorname{vector}(1$, NNP);

$\mathrm{yy}=\operatorname{vector}(1, \mathrm{MMP})$;

ww $=\operatorname{vector}(1$, MMP);

d_ $\mathrm{x}=\mathrm{DX} * \mathrm{NCX} / \mathrm{NFX}$

d_y $=$ DY;

/* computation of distorted image coordinate */

for ( $i=1 ; i<-p n u m$; $i++)$ (

$\mathrm{Xd}[\mathrm{i}]=\mathrm{d}_{-} \mathrm{x} *(\mathrm{xf}[\mathrm{i}]-\mathrm{CX}) / \mathrm{sX}$;

$\mathrm{Yd}[1]=\mathrm{DY} *(\mathrm{yf}[\mathrm{i}]-\mathrm{CY})$;

\}

/* solve for first set of variables */

for ( $i-1$; i<-pnum ; $i++)$ )

aa $[i][1]=\operatorname{Yd}[\mathbf{i}] * x w[i]$;

aa[i][2] $=$ Yd[i]*yw[i];

aa[i][3] - Yd[i];

aa $[i][4]=-X d[i] * x w[i]$

aa $[\mathbf{i}][5]=-X d[i] * y w[i]$

$\mathrm{bb}[\mathbf{i}]=\mathrm{Xd}[\mathbf{i}]$; 
$\mathrm{n}=\mathbf{3}$

EQSOLVE ( $a \mathrm{a}, \mathrm{pnum}, \mathrm{n}, \mathrm{bb}, \mathrm{xx}$ ) ;

Ty_1r1 - xx[1];

Ty_1r2 $=x x[2]$

Ty_1Tx $=\mathrm{xx}[3]$;

Ty_1r4 - xx[4];

Ty_1r5 $=\mathrm{xx}[5]$;

/* compute ABS ( Ty ) */

r_1 - Ty_lr1;

$\mathrm{r}_{-} 2$ - Ty_lr2;

$\mathrm{r}_{-} 4$ - Ty_1r4;

r_5 = Ty_1r5;

/* introduce c - matrix [ $\left.r_{-} 1 r_{-} 2 ; r_{-} 4 r_{-} 5\right] * /$

if $\left(\left(\left(r_{-} 1-0\right) \delta \&\left(r_{-} 2^{-}-\overline{0}\right)\right)\right.$ or

$\left(\left(r_{-} 4-0\right)\right.$ d\& $\left.\left(r_{-}-0\right)\right)$ or

$\left(\left(r_{-} 1-0\right) \& \&\left(r_{-}-0\right)\right)$ or

$\left(\left(r_{-}^{-}-0\right)\right.$ \&\& $\left.\left.\left(r_{-}^{-}-0\right)\right)\right)($

/* if a whole row or column of martix c vanishes */

if $\left(\left(r_{-} 1-0\right)\right.$ \&\& $\left.\left(r_{-} 2-0\right)\right)$

( $r i=\bar{r}_{-} 4 ;$ rj-r_5; $\left.\operatorname{err} \overline{1}++;\right)$;

if $\left(\left(r_{-} \overline{4}-0\right)\right.$ d\& $\left.\left(r_{-} 5-0\right)\right)$

( ri-r_1; rj=r_2; err $\overline{1}++;)$;

if $\left(\left(r_{-} \overline{1}-0\right)\right.$ \&\& $\left.\left(r_{4}-0\right)\right)$

( $\left.r i=\bar{r}_{2} 2 ; r j=r \quad 5 ; \operatorname{err} \overline{1}++;\right)$;

if $\left(\left(r_{-} \overline{2}-0\right)\right.$ \&\& $\left.\left(r_{-} 5-0\right)\right)$

( $r i=\bar{r}_{-} 1 ; \quad r j=r_{-} 4 ;$ err $\left.\overline{1}++;\right)$;

if $(\operatorname{err} 1-\overline{1}) \mathrm{T} 2 \mathrm{y}=1 /(\mathrm{ri} * \mathrm{ri}+\mathrm{rj}$ *rj );

else printf("c - matrix double zero error");

/* if NOT a whole row or column of martix c vanishes */ else 1

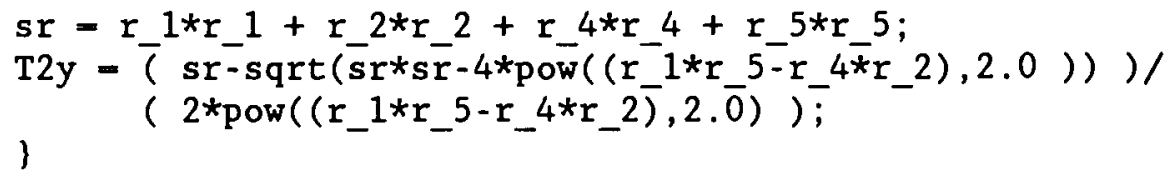

Ty $=\operatorname{sqrt}(\mathrm{T} 2 \mathrm{y})$

$/ *$ determine the sign of Ty */

$1 *$ use a point whose computer image coordinates is away */

$/ *$ from the center */

Xddist $=\mathrm{Xd}[$ pnum $]$;

Yddist $=$ Yd[pnum ] ;

Xwdist - xw[pnum];

Ywdist - yw[pnum]; 


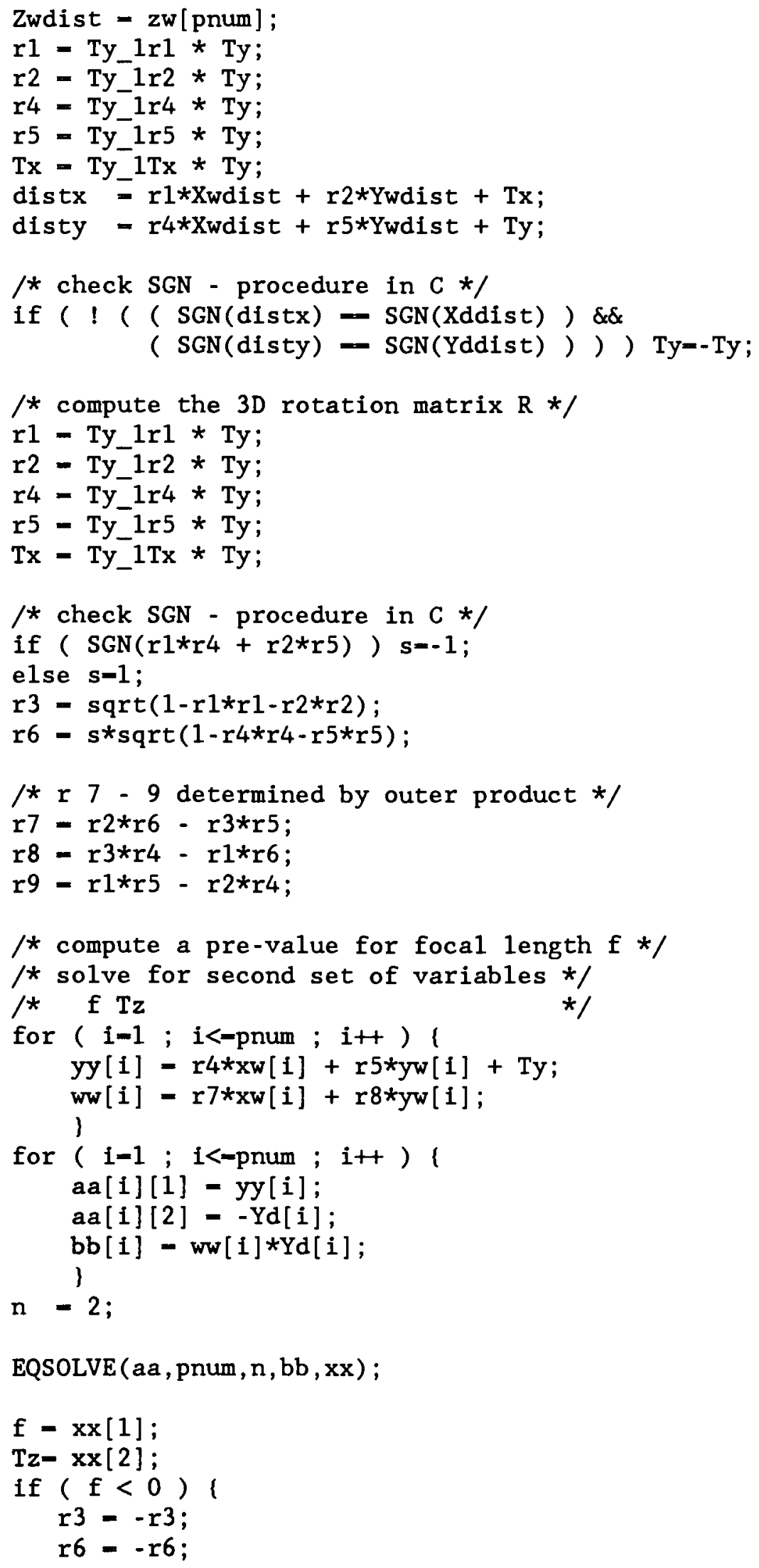




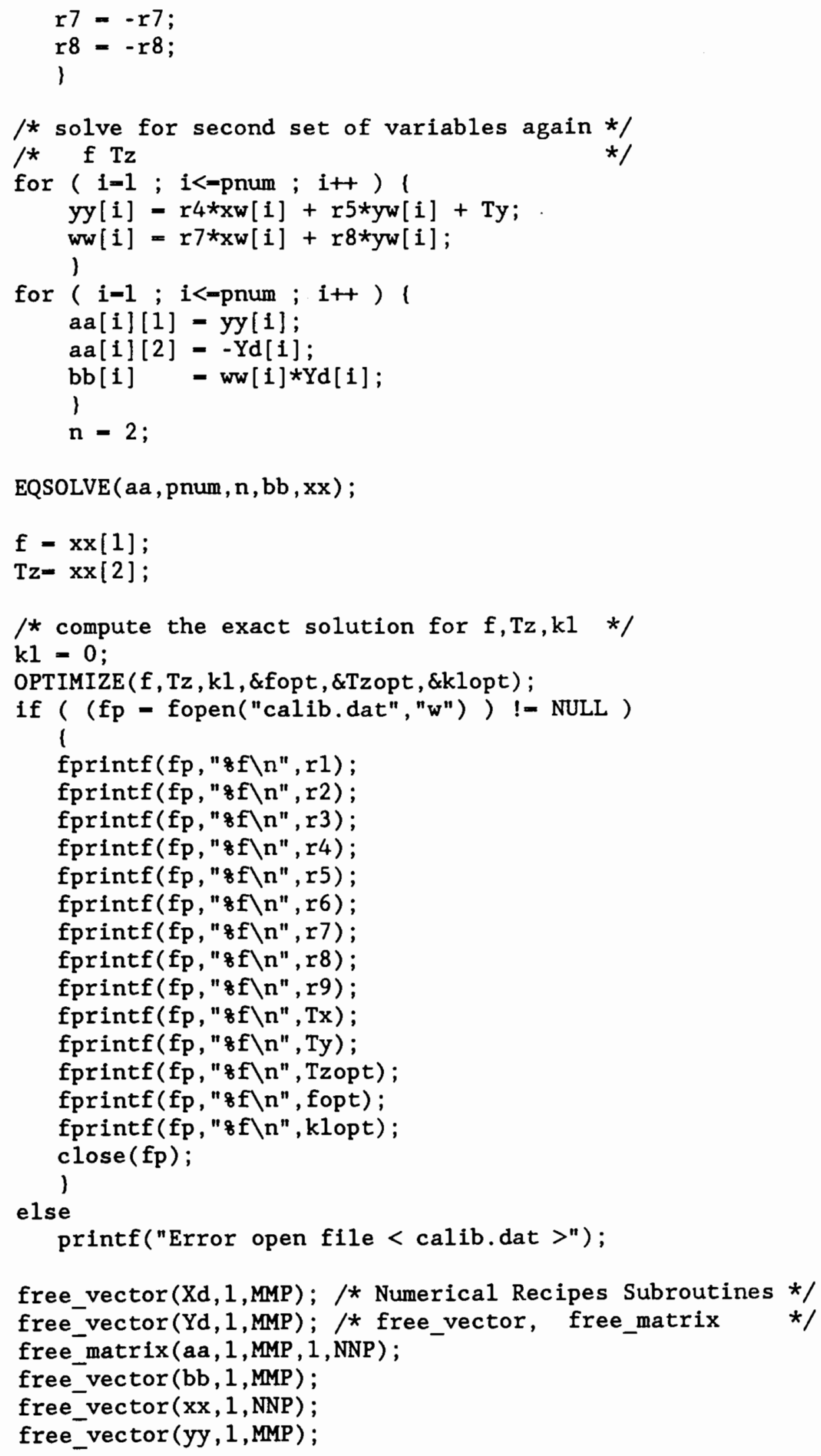




\footnotetext{
free_vector(ww, 1,MMP);

$1 / *$ end of CALIB *
}

float EQSOLVE (aa, $\mathrm{m}, \mathrm{n}, \mathrm{bb}, \mathrm{xx})$

float $* * a a, * b b, * x x$;

int $\mathrm{m}, \mathrm{n}$;

/* Subroutine - EQSOLVE

/* Solves overdetermined set of linear algebraic */

/* equations using singular value decomposition */

/* Numerical Recipes, Press, Flannery, Teuko1sky */

$/ *$ Vetterling

\#define MP 50

\#define NP 20

int $i, j$;

float $* w, * x, * b, * * a, * * u, * * v$;

float wmin, wmax;

w $=\operatorname{vector}(1, N P)$;

$\mathrm{x}=\operatorname{vector}(1, \mathrm{NP})$;

$\mathrm{b}=\operatorname{vector}(1, \mathrm{MP})$;

$a=\operatorname{matrix}(1, M P, 1, N P)$;

$u=\operatorname{matrix}(1, \mathrm{MP}, 1, \mathrm{NP})$;

$\mathrm{v}=\operatorname{matrix}(1, \mathrm{NP}, 1, \mathrm{NP})$;

for $(i=1 ; i<-m ; i++)($

for $(j=1 ; j<-n ; j+)($

$a[i][j]=a a[i][j]$;

)

)

for $(i=1 ; i<-m ; i+) b[i]-b b[i]$;

for $(i=1 ; i<-m ; i+t)$ (

for $(j=1 ; j<-n ; j++)$

$u[\mathbf{i}][\mathbf{j}]=a[\mathbf{i}][\mathbf{j}]$;

)

)

$\operatorname{svdcmp}(u, m, n, w, v) ; / *$ Numerical Recipes Subroutine */

/ * find maximum singular value */

wmax $=0.0$;

for $(j=1 ; j<-n ; j+)$

if $(w[j]>$ wmax $)$ wmax $=w[j]$; 


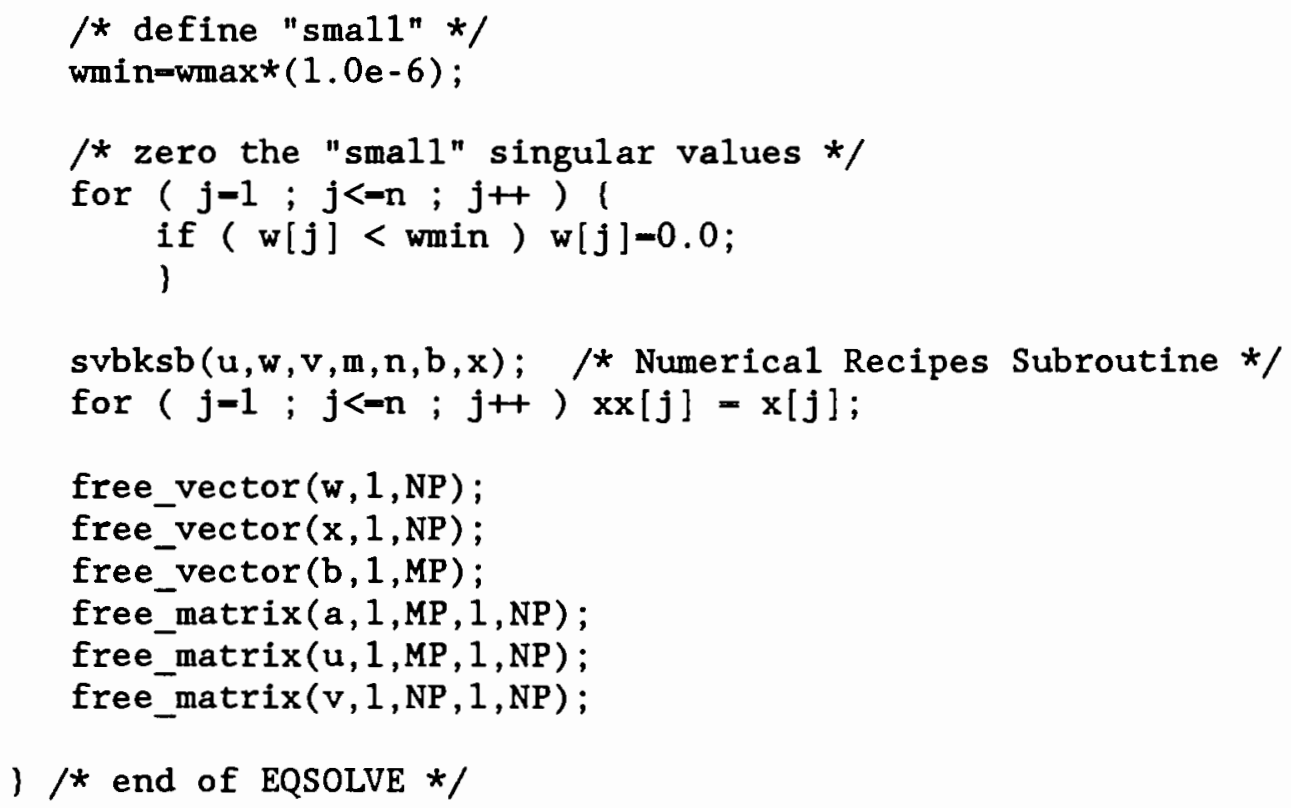

int 1 ;

float part1, rsqr, num, den, part2, sum;

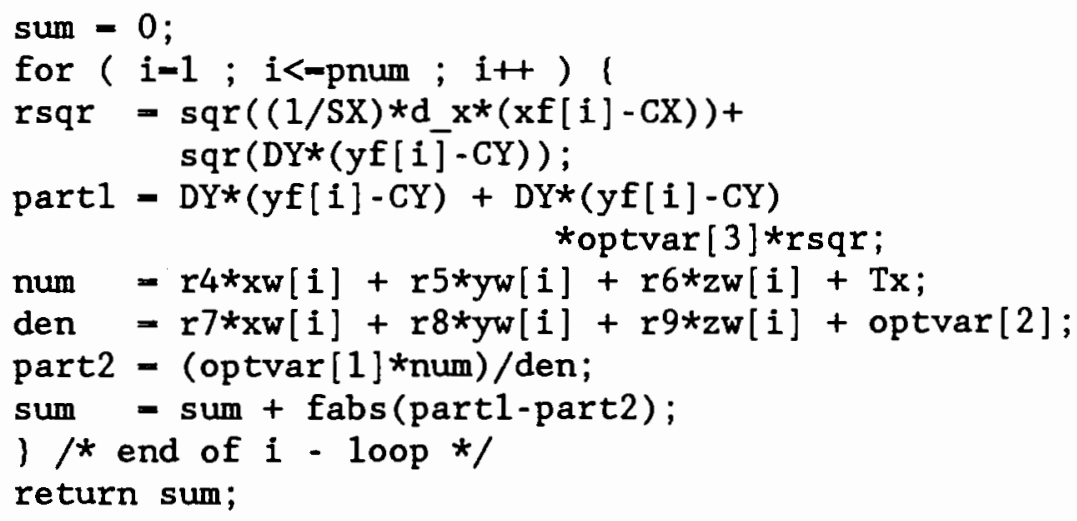

$1 *$ end of func $* /$ 
void dfunc (optvar, df)

float optvar[], df [];

(

int $i$;

float part1, rsqr, num, den, part2, sum;

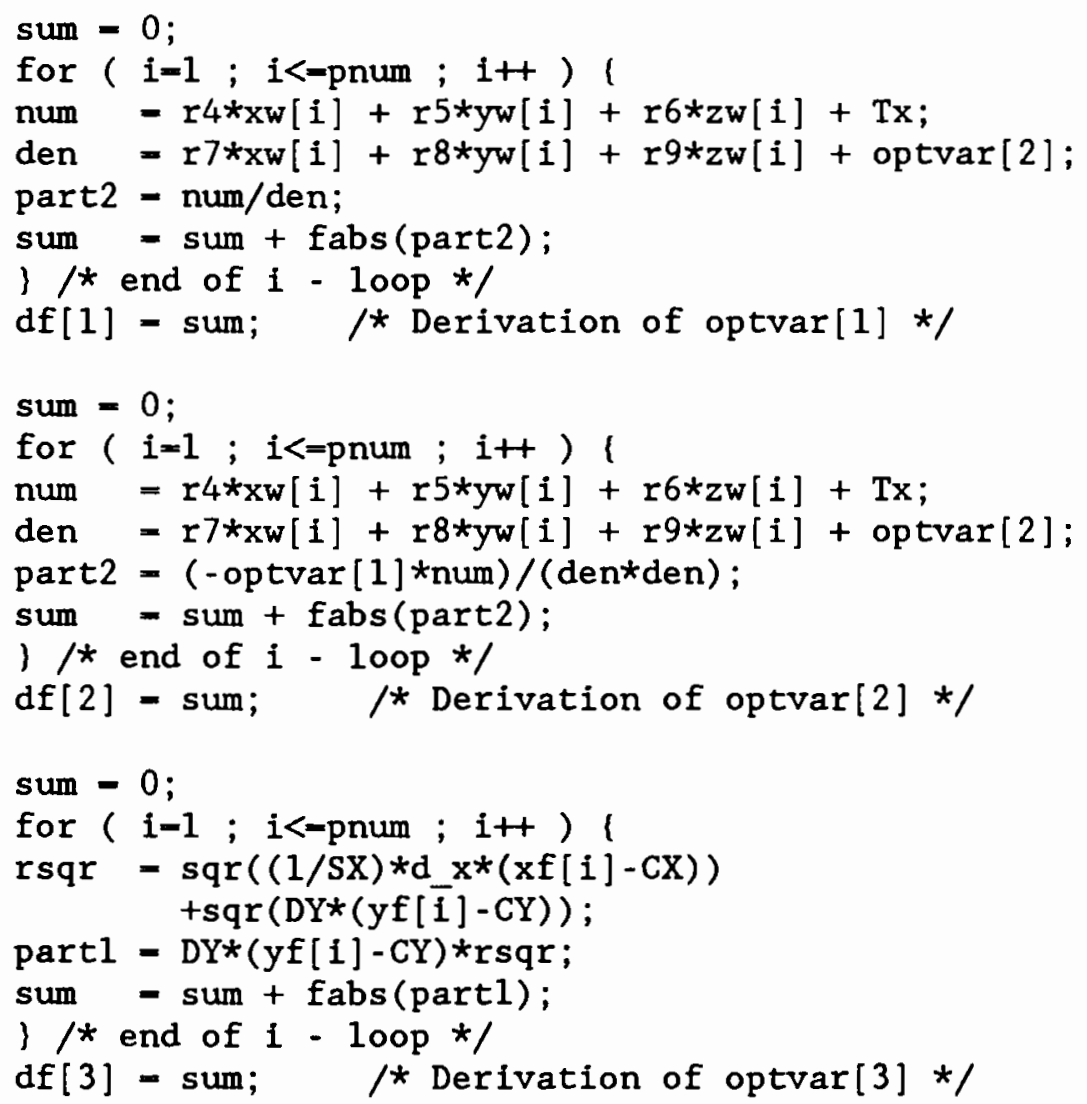

\} $/ *$ end of dfunc */

OPTIMIZE ( $f, T z, k 1$, fopt, Tzopt, klopt)

float $\mathrm{f}, \mathrm{Tz}, \mathrm{kl}, *$ fopt, $* \mathrm{Tzopt}, \star_{\mathrm{klopt}}$

/* Subroutine - OPTIMIZE
/* Optimization by Conjugate Gradient Methods
/* Polak-Ribiere variant
/* Numerical Recipes, Press, Flannery, Teukolsky
/* Vetterling

1

int iter;

float fret, ${ }^{*} p$;

p-vector ( 1, NDIM);

$p[1]-f$; 


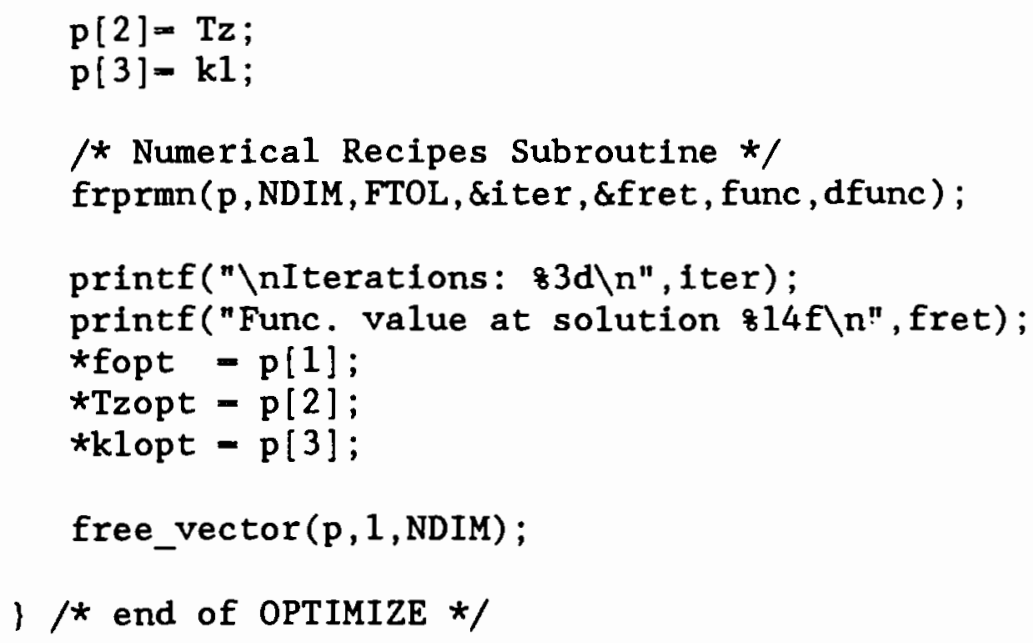


APPENDIX G

THE INPUT PARAMETERS FOR EACH METHOD AND SIMULATION CASE 
Low Contrast
Simulation

Threshold(a) Method: Threshold

Threshold(b) Method: Lowcut

Roberts Edge Filter:

Variance (3) Method:

Option 1
Highcut

Gradient

Threshold

90

77

103

24

380

Variance

Threshold

Lowcut

Highcut

Variance

Threshold

Lowcut

Highcut

Threshold

Lowcut

Highcut
High Contrast Simulation

140

115

165

49

1500

65

215

3500

65

215

140

65

215

Bandwidth for Local Methods with Option 2: 10 Pixels 